\title{
Investigation and development of post-season modelling of Arrow squid in the Snares and Auckland Islands
}

\author{
by \\ Vidette Louise McGregor \\ A thesis \\ submitted to Victoria University of Wellington \\ in partial fulfilment of the \\ requirements for the degree of \\ Master of Science \\ in Statistics and Operations Research
}

Victoria University of Wellington

2013 


\section{Abstract}

Squid fisheries require a different management approach to most fish species which are much longer living. Most squid live for around one year, spawn and then die. The result of this is an entirely new stock each year with little or no relationship of stock sizes between the years. Hence, it is difficult to set appropriate catch limits prior to the season.

Currently, there is nothing set up for modelling the New Zealand squid fishery in-season or post-season. In-season management would allow for adjustments of catch limits during a season. Post-season management would provide information on how much the stock was exploited during a season (described as the escapement).

I have produced an integrated model using ADMB (Automatic Differentiation Model Builder) (Fournier et al., 2011) which models length frequency data, CPUE (Catch Per Unit Effort) indices and catch weights from a season. It calculates escapement which indicates how much the fishery is currently being exploited.

In running the model against data from four area and year combinations, I found the escapement calculation to be stable. The results suggest this modelling approach could be used with the current data collected for post-season modelling of the fishery.

I am less confident about in-season modelling with the current data collected. The integrated model fits quite poorly to the CPUE data, suggesting some discrepancy either between the data or the assumptions made of them. Sampling from a greater number of tows is recommended to improve the length frequency data and this may also improve the ability of the model to fit both to these and the CPUE. 


\section{Acknowledgements}

I have great pleasure in thanking:

- Shirley Pledger of Victoria University and Chris Francis of NIWA for supervising the thesis

- The Ministry of Fisheries for the post graduate scholarship

- NIWA for providing me with an office, a supervisor and unlimited cups of tea

- Rosie Hurst for coming up with the thesis topic

- Dan Fu for helping me set up ADMB on my computer

- My family - my wonderful and supportive partner Ben and my daughter Sophia and son Marcus for being understanding when I have spent so many hours at work

- Our cats Kush and the late Smeagol for being supportive and understanding

- Mum and Dad for being so proud 


\section{Contents}

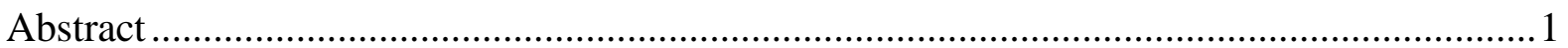

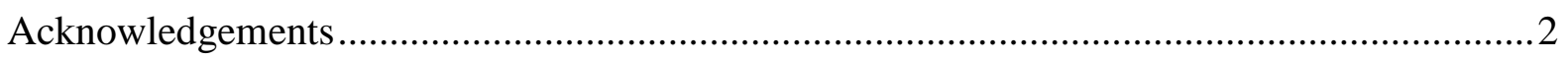

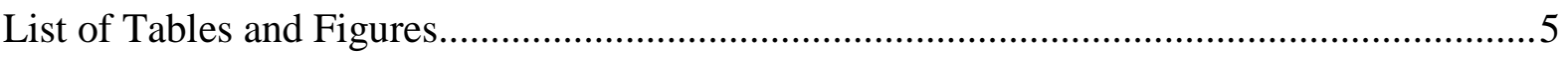

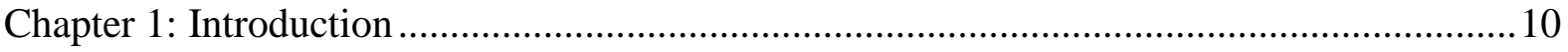

Chapter 2: In-season management and post-season assessment modelling............................12

Chapter 3: The fishery - Snares and Auckland Islands .......................................................14

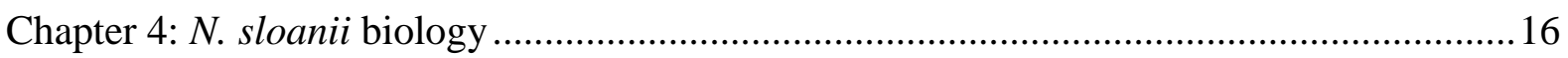

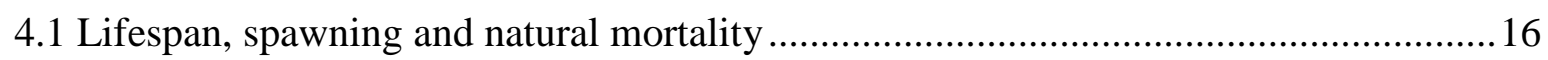

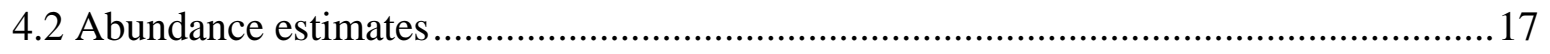

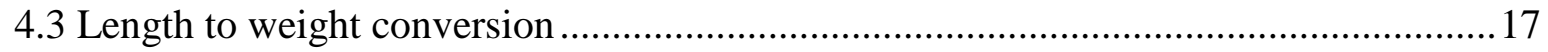

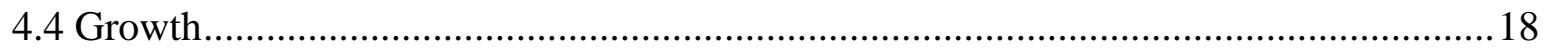

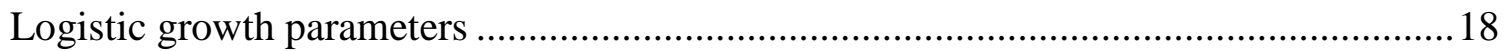

Expected growth as a function of length ................................................................. 19

Expected growth with estimated parameters ............................................................ 21

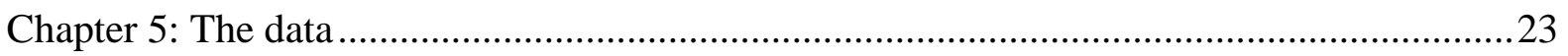

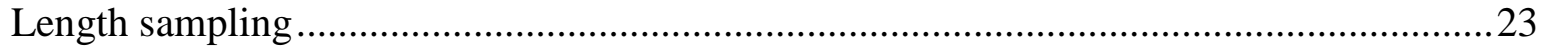

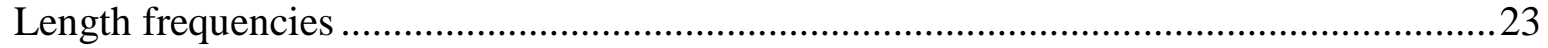

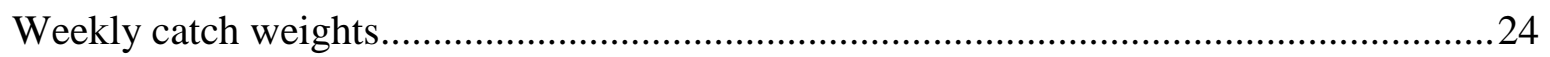

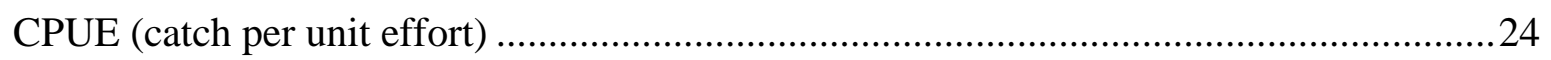

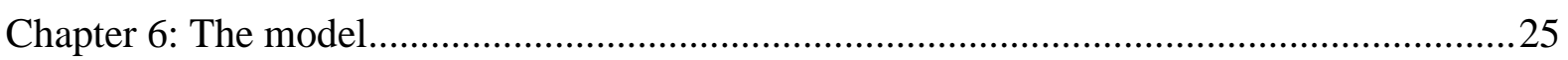

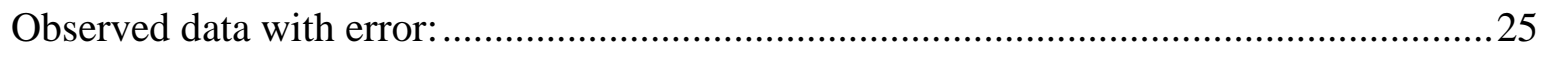

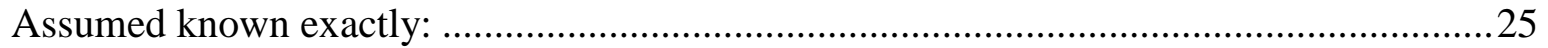

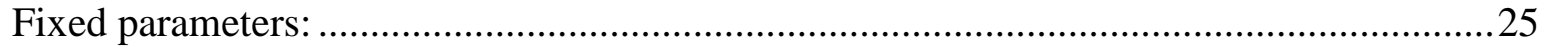

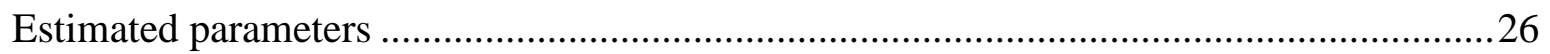

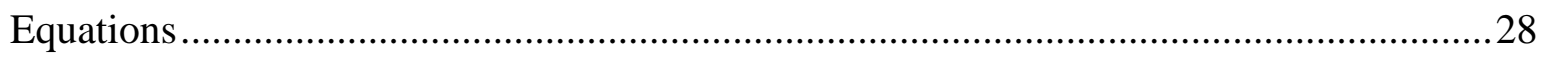

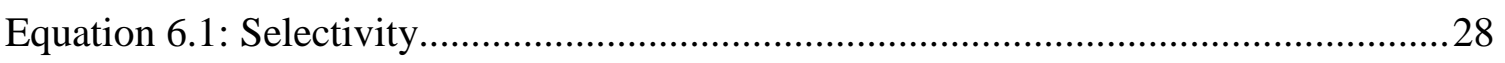

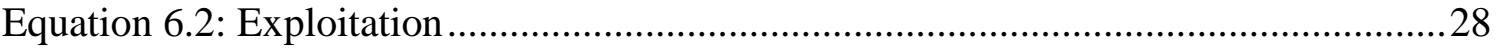

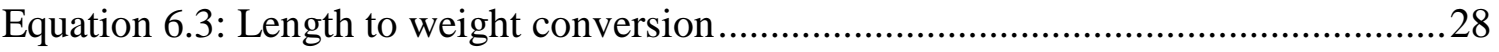

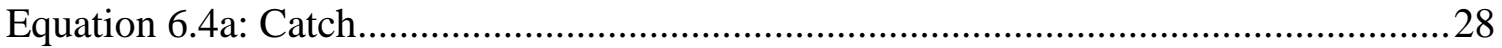

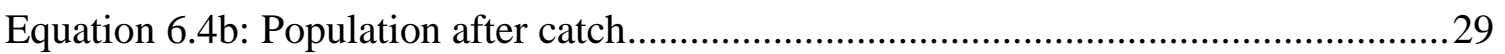

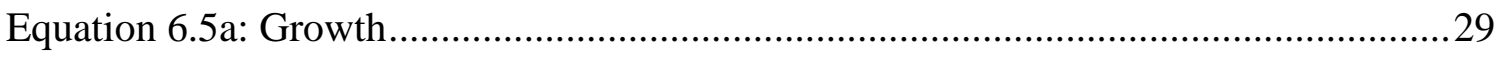

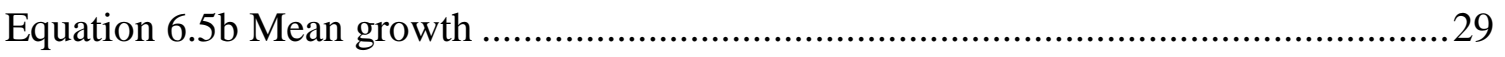

Equation 6.6: Length distribution at recruitment.......................................................... 30

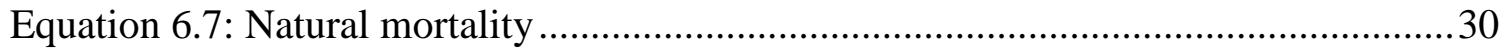


Equation 6.8: Likelihood for proportions at length (Fournier et al., 1990) .......................30

Equation 6.9: CPUE likelihood (Bull et al., 2008) ....................................................... 31

Equation 6.10: Calculating $\mathrm{q}$ as a nuisance parameter (Bull et al., 2008) ........................31

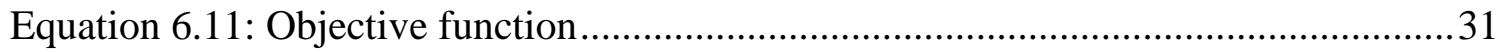

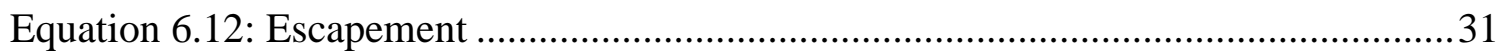

Equation 6.13: Variance of standardised difference of mean lengths ............................ 31

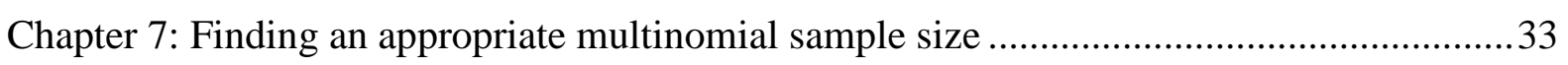

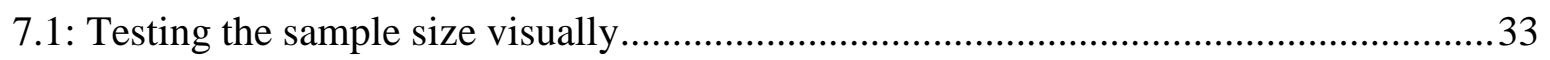

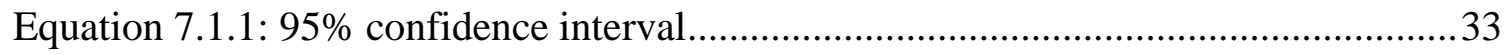

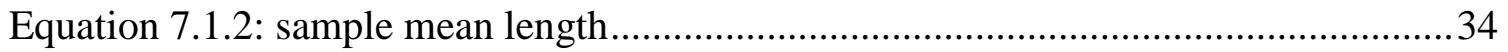

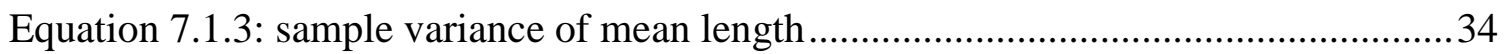

7.2: Calculating the variance of the standardised error of mean lengths ............................34

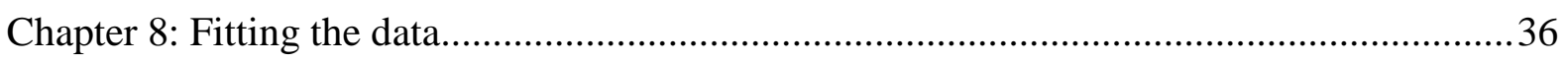

8.1: How many cohorts and what shape function for mean growth? .................................36

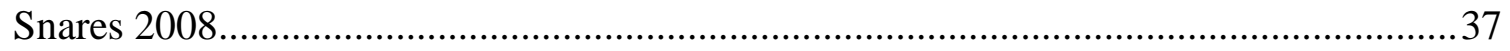

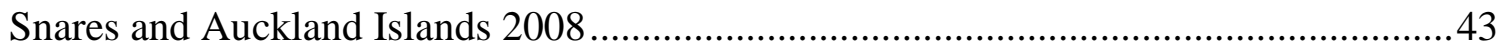

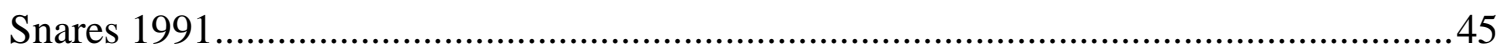

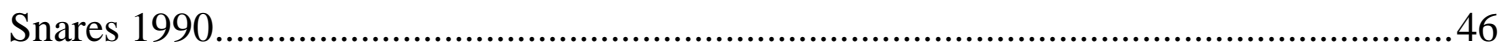

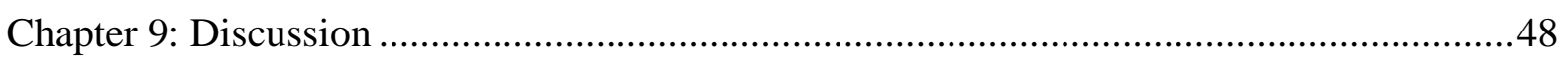

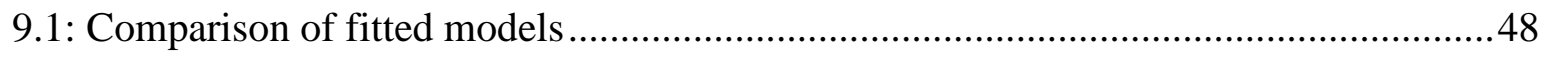

9.2: The modelling approach used and future recommendations.....................................49

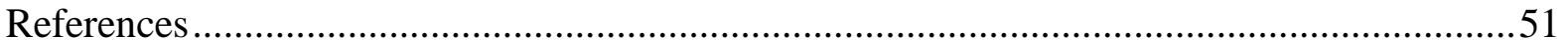

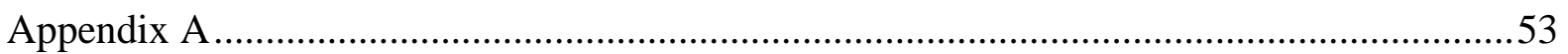

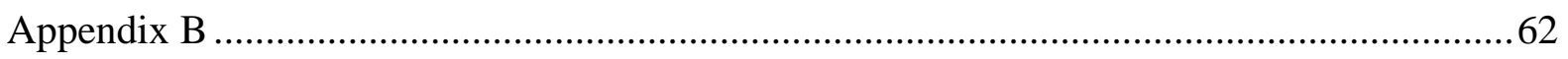

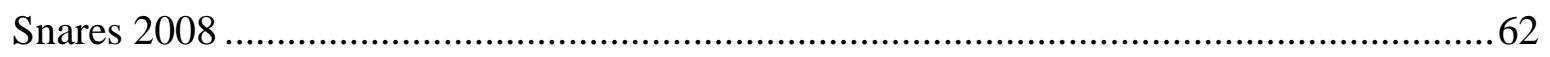

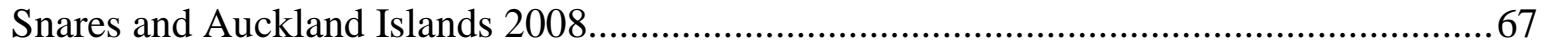

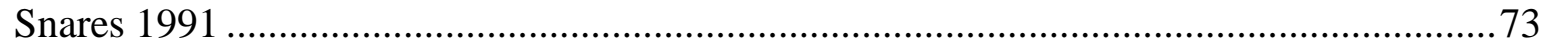

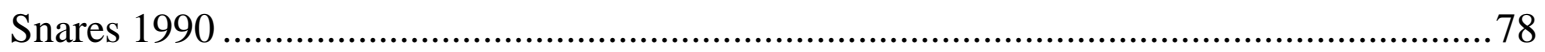

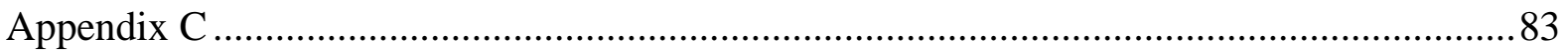




\section{List of Tables and Figures}

Figure 3a: The position of the Subtropical Convergence (Kowalski \& A. Meyers, 1997). ....14

Figure 3b: The NZ EEZ and QMAs (Quota Management Areas) for squid. ......................... 14

Table 3a: Reported catches (t) and TACCs (t) of arrow squid from 2001-02 to 2009-10

(Ministry of Fisheries, 2011)

Figure3c: Map showing fishing areas PUYS (Puysegur), STEW (Stewart Islands), SNAR

(Snares) and AUCK (Auckland Islands).....

Figure 2a: Length to weight conversion curves for Snares and Auckland Islands.....

Table 2a: Estimated growth parameters for N. sloanii, by month of hatching (Uozumi 1998).

Table 2b: Estimated growth parameters for $N$. sloanii, averaged over relevant months for female, male and combined sexes.

Figure 2b: Expected length vs age for combined sexes, with mean parameters from relevant spawning months.

Figure 2c: Instantaneous growth rate using combined average Uozumi parameters (Table 2b).

Figure 2d: Weekly growth using combined average Uozumi parameters (Table 2b).

Figure 2d: Examples of expected mean growth using constant, linear and broken stick equations, compared to Uozumi's growth parameters.

Table 5.1: Number of tows sampled from for each week, for each area and year combination.

Table 6a: Data and parameters used in the models ..............................................................25

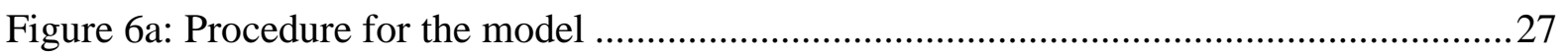

Figure 7.1: Observed mean length $(*)$. The vertical lines are $95 \%$ confidence intervals, calculated assuming simple random sampling: a) $\mathrm{N}=100$, b) $\mathrm{N}=25$. Plots produced for the purpose of illustration only.

Figure 8.1a: Schematic showing a new recruitment cohort coming into the total population and the selected (vulnerable part of the) population from which the catch is taken. The colour of the line refers to the total or selected population. The type of line indicates the existing population, the new recruitment and the resulting population from adding the new recruitment to the existing population.

Table 8.1.1a: Calculated objective function and variance of standardised error of mean lengths for model fitted with 1, 2, and 3-cohorts and constant, linear and broken stick mean growth for Snares 2008 .

Table 8.1.1b: Timing and size of cohorts for models fitted with 1,2, and 3-cohorts and constant, linear and broken stick mean growth for Snares 2008.

Table 8.1.1c: Estimated parameters for Snares 2008 models with 1, 2, and 3-cohorts and constant, linear and broken stick mean growth for Snares 2008.

Figure 8.1.1a: Expected mean growth per week by length from models with 1, 2, and 3cohorts and constant, linear and broken stick mean growth for Snares 2008. The colour of each line indicates the mean growth function and the type of line indicates the number of cohorts. The dotted lines are for the 3-cohort models. The dashed lines are the 2-cohort models. Note that linear and broken stick 2-cohort models share the same line. The solid lines are the 1-cohort models. 
Figure 8.1.1b: Observed CPUE compared to expected CPUE for models with 1, 2, and 3cohorts and constant, linear and broken stick mean growth for Snares 2008.

Figure 8.1.1c: Observed mean lengths with $95 \%$ confidence intervals, compared to expected mean lengths for models with 1,2, and 3-cohorts and constant, linear and broken stick mean growth for Snares 2008. The vertical black lines show the 95\% confidence intervals for the observations, calculated using the number of tows (the numbers above the black lines) as the actual sample sizes. The asterisks are the mean lengths from the data......

Figure 8.1.1d: Observed length frequencies compared to expected length frequencies for models with 1, 2, and 3-cohorts and constant (1), linear (2) and broken stick (3) mean growth for Snares 2008.

Figure 8.1.2a: Observed mean lengths with $95 \%$ confidence intervals, compared to expected mean lengths for Snares and Auckland Islands 2008, for 1, 2, and 3 mean growth parameters and 2-cohorts. The vertical black lines show the $95 \%$ confidence intervals for the observations, calculated using the number of tows (the numbers above the black lines) as the actual sample sizes.

Figure 8.1.2b: Observed length frequencies against expected length frequencies for Snares and Auckland Islands 2008, for 1, 2, and 3 mean growth parameters and 2-cohorts. Figure 8.1.2c: Expected mean growth by length for Snares and Auckland Islands 2008 from models with 1,2, and 3 mean growth parameters and 2-cohorts. Mean growth is the mean growth for 1 week.

Figure 8.1.3a: Observed mean lengths with $95 \%$ confidence intervals, compared to expected mean lengths for Snares 1991, for constant, linear, and broken stick mean growth and 3cohorts. The vertical black lines show the $95 \%$ confidence intervals for the observations, calculated using the number of tows (the numbers above the black lines) as the actual sample sizes.

Figure 8.1.3: Observed CPUE compared to expected q×exploitable biomass for Snares 1991, for 1, 2, and 3 mean growth parameters and (a) 1-cohort (b) 3-cohorts. Note, that the linear growth and broken stick growth models share the same line, hence only the broken stick line is visible.

Figure 8.1.4a: Observed mean lengths with $95 \%$ confidence intervals, compared to expected mean lengths for Snares 1990, for constant, linear and broken stick mean growth and 2cohorts. The vertical black lines show the $95 \%$ confidence intervals for the observations, calculated using the number of tows (the numbers above the black lines) as the actual sample sizes.

Figure 8.1.4b: Observed CPUE compared to expected q Xexploitable biomass for Snares 1990, for constant, linear and broken stick mean growth and 2-cohorts.

Table 9.1a: Expected parameter values from 'best' models as selected in section 8.1 for the four area/year combinations; Snares 2008, 1991, 1990 and Snares and Auckland Islands combined 2008.

Figure A1a: Length frequency of arrow squid taken in target arrow squid commercial catches from the SNARES SHELF trawl fishery 1990 sampled by the Observer Programme. n, number of tows sampled; no., number of fish sampled (Hurst et al., 2012).

Figure A1b: Length frequency of arrow squid taken in target arrow squid commercial catches from the SNARES SHELF trawl fishery 1991 sampled by the Observer Programme. n, number of tows sampled; no., number of fish sampled (Hurst et al., 2012)........................54 Figure A1b continued: SNARES SHELF trawl fishery 1991 length frequencies ...................55 
Figure A1d: Length frequency of arrow squid taken in target arrow squid commercial catches from the SNARES SHELF trawl fishery 2008 sampled by the Observer Programme. n, number of tows sampled; no., number of fish sampled (Hurst et al., 2012).......................56 Figure A1d continued: SNARES SHELF trawl fishery 2008 length frequency. ....................57 Figure A1e: Length frequency of arrow squid taken in target arrow squid commercial catches from the AUCKLAND ISLANDS trawl fishery 2008 sampled by the Observer Programme. n, number of tows sampled; no., number of fish sampled (Hurst et al., 2012).......................58 Figure A1e continued: AUCKLAND ISLANDS trawl fishery 2008 length frequency..........59 Table A2a: Snares CPUE estimated values (arbitrary units) and CVs for the lognormal, target squid by week for the fishing years 1990, 1991 and 2008 (Hurst et al. 2012).

Table A2b: Auckland Islands CPUE estimated values (arbitrary units) and CVs for the lognormal, target squid by week for the fishing year 2008 (Hurst et al., 2012).

Table A2c: Catch weights ( $t$ ) for all vessels for each week for selected years (Hurst et al.

2012).

Table B.1a: Calculated objective function and variance of standardised error of mean lengths for model fitted with 1, 2, and 3-cohorts and constant, linear and broken stick mean growth for Snares 2008.

Table B.1b: Timing and size ofcohorts for models fitted with 1, 2, and 3-cohorts and constant, linear and broken stick mean growth for Snares 2008.

Table B.1c: Estimated parameters for Snares 2008 models with 1, 2, and 3-cohorts and constant, linear and broken stick mean growth for Snares 2008.

Figure B.1a: Expected mean growth per week by length from models with 1, 2, and 3-cohorts and constant, linear and broken stick mean growth for Snares 2008. The colour of each line indicates the mean growth function and the type of line indicates the number of cohorts......63 Figure B.1b: Observed CPUE compared to expected CPUE for models with 1, 2, and 3cohorts and constant, linear and broken stick mean growth for Snares 2008.

Figure B.1c: Observed mean lengths with 95\% confidence intervals, compared to expected mean lengths for models with 1, 2 and 3-cohorts and constant, linear and broken stick mean growth for Snares 2008. The vertical black lines show the 95\% confidence intervals for the observations, calculated using the number of tows (the numbers above the black lines) as the actual sample sizes. The asterisks are the mean lengths from the data.....

Figure B.1d: Observed length frequencies compared to expected length frequencies for models with 1, 2 and 3-cohorts and constant, linear and broken stick mean growth for Snares 2008

Table B.2a: Calculated objective function and variance of standardised error of mean lengths for model fitted with 1, 2, and 3-cohorts and constant, linear and broken stick mean growth for Snares and Auckland Islands 2008.

Table B.2b: Timing and size of cohorts for models fitted with 1, 2, and 3-cohorts and constant, linear and broken stick mean growth for Snares and Auckland Islands 2008.

Table B.2c: Expected parameters for Snares and Auckland Islands 2008 models with constant, linear and broken stick mean growth and 1, 2, and 3-cohorts.

Figure B.2a: Expected mean growth by length for Snares and Auckland Islands 2008 from models with constant, linear and broken stick mean growth and 1, 2 and 3-cohorts. Mean growth is the mean growth for 1 week.

Figure B.2b: Observed CPUE compared to expected q $\times$ exploitable biomass for Snares and Auckland Islands 2008, for constant, linear and broken stick mean growth and 1, 2 and 3cohorts. 
Figure B.2c: Observed mean lengths with 95\% confidence intervals, compared to expected mean lengths for Snares and Auckland Islands 2008, for constant, linear and broken stick mean growth and 1,2 and 3-cohorts. The vertical black lines show the $95 \%$ confidence intervals for the observations, calculated using the number of tows (the numbers above the black lines) as the actual sample sizes.

Figure B.2d: Observed length frequencies compared to expected length frequencies for Snares and Auckland Islands 2008, for constant, linear and broken stick mean growth and 1, 2 and 3-cohorts. The last set of graphs show the constant growth (1 mean growth parameter) model with 1, 2 and 3-cohorts.

Figure B.2d: (continued)

Table B.3a: The calculated objective function and variance of the standardised error of mean lengths for models fitted with 1,2, and 3-cohorts and constant, linear and broken stick mean growth for Snares 1991

Table B.3b: Timing and size of cohorts for models fitted with 1, 2, and 3-cohorts and constant, linear and broken stick mean growth for Snares 1991.

Table B.3c: Expected parameters for Snares 1991 models with constant, linear and broken stick mean growth and 1,2, and 3-cohorts.

Figure B.3a: Expected mean growth by length for Snares 1991 from models with constant, linear and broken stick mean growth and 1,2, and 3-cohorts. Mean growth is the mean growth for 1 week.

Figure B.3b: Observed CPUE compared to expected q×exploitable biomass for Snares 1991, for constant, linear and broken stick mean growth and 1,2 and 3-cohorts.

Figure B.3c: Observed mean lengths with $95 \%$ confidence intervals, compared to expected mean lengths for Snares 1991, for constant, linear and broken stick mean growth and 1, 2 and 3 -cohorts. The vertical black lines show the $95 \%$ confidence intervals for the observations, calculated using the number of tows (the numbers above the black lines) as the actual sample sizes.

Figure B.3d: Observed length frequencies compared to expected length frequencies for Snares 1991, for constant, linear and broken stick mean growth and 1, 2 and 3-cohorts........77 Table B.4a: The calculated objective function and the variance of the standardised error of mean lengths for model fitted with 1,2 and 3-cohorts and constant, linear and broken stick mean growth for Snares 1990.

Table B.4b: Timing and size of cohorts for models fitted with 1, 2, and 3-cohorts and constant, linear and broken stick mean growth for Snares 1990.

Table B.4c: Expected parameters for Snares 1990 models with constant, linear and broken stick mean growth and 1,2, and 3-cohorts.

Figure B.4a: Expected mean growth by length for Snares 1990 from models with constant, linear and broken stick mean growth and 1,2, and 3-cohorts. Mean growth is the mean growth for 1 week.

Figure 8.3.2a: Observed CPUE compared to expected q $\times$ exploitable biomass for Snares 1990, for constant, linear and broken stick mean growth and 1, 2 and 3-cohorts.

Figure 8.3.2b: Observed mean lengths with $95 \%$ confidence intervals, compared to expected mean lengths for Snares 1990, for constant, linear and broken stick mean growth and 1, 2 and 3 -cohorts. The vertical black lines show the $95 \%$ confidence intervals for the observations, calculated using the number of tows (the numbers above the black lines) as the actual sample sizes. 
Figure 8.3.: Observed length frequencies compared to expected length frequencies for Snares 1990, for 1, 2, and 3 mean growth parameters and 1, 2 and 3-cohorts. Last plot shows the constant growth ( 2 mean growth parameters) model with 1,2 and 3 -cohorts........................ 82 


\section{Chapter 1: Introduction}

Effective management of a squid fishery requires an approach different from that used for most fish species, which are much longer living. Squid generally live for around one year, spawn and then die (Ministry of Fisheries, 2011). Hence, any information on the stock in one year has little to no relevance to the stock of any other year, except in the extreme case where the fishery catches the entire stock before it is able to spawn. In particular, it does not give us the information required to set an appropriate catch limit prior to the commencement of a season.

The New Zealand squid fishery is currently managed by a set TACC (Total Allowable Commercial Catch). Setting a TACC is extremely difficult in the case of short-lived species such as squid, unless real-time monitoring of the resource biomass is feasible (Augustyn \& Roel, 1998). Without the information required to support adjusting this limit mid-season (inseason management), the fishery runs the risk of over-or underexploiting its stock. Currently, there is also no post-season modelling of the fishery to indicate whether any previous seasons are likely to have under-or over exploited the stock.

This thesis aims to determine whether post-season modelling is possible with the data currently collected from the fishery. A successful outcome of this will be an estimated rate of escapement for each season. This is a proportion, calculated as the expected population at the conclusion of the fishing season over the expected population at the same time had there been no fishing for the entire season. Depletion (1-escapement) and escapement rates have been used for in-season management and commonly aim to ensure $40 \%$ of the estimated biomass will survive to spawn (Beddington et al., 1990). Depending on the success of this post-season modelling, it may be possible to give some indication as to the feasibility of in-season modelling for future seasons using current or modified data collection methods.

The modelling approach used here is an "integrated model". In the fisheries literature, this connotes an approach that is capable of using a wide variety of types of observation, and always treats them statistically (Maunder \& Punt, 2004). The benefits of this approach in contrast to some others used for squid fisheries outside New Zealand are discussed in Chapter 9. The other approaches include depletion methods (Young et al., 2004), cohort analysis and modified cohort analysis (Royer et al., 2002) and Bayesian assessments (McAllister et al., 2004).

Benefits of in-season and post-season management for a squid fishery are covered in Chapter 2, with examples of approaches from other squid fisheries outside New Zealand. The justification for considering these approaches for the New Zealand squid fishery is given.

Chapter 3 gives an overview of the New Zealand squid fishery including how it is managed, where the majority of the catches are coming from and how big the catches are. This research will focus on the Snares fishery which makes up a considerable proportion of the New Zealand squid fishery. The species caught in the Snares fishery is Nototodarus sloanii.

The relevant biology of $N$. sloanii is covered in Chapter 4, including lifespan, spawning, natural mortality, abundance estimates, length to weight conversion and growth. 
The data used for this thesis are from the analysis carried out by Hurst et al. (2012). They are length frequencies, CPUE (Catch Per Unit Effort) indices and catch weights. Descriptions of these data are in Chapter 5.

The model developed to estimate escapement uses four processes to explain the data; new recruitment cohorts come into the modelled population, the squid grow, suffer natural mortality and are subjected to fishing mortality. Chapter 6 explains how these processes make up the model, includes a table of data and parameters used, a flowchart of the processes as they occur within each time-step (week) and the equations.

In fitting this model to the data, there are two main questions to answer: how many recruitment cohorts are required and what shape is the function for the mean growth. Section 8.1 explains how to best answer these questions by analysing the fit of the models to the data. A full analysis of the Snares 2008 fishing season is then given, followed by a brief summary analysis for the remaining seasons. Chapter 8.2 compares the estimated parameters from the 'best' models as selected.

Chapter 9 discusses the model and its suitability for the current data. The models selected in Chapter 8 and the results of these are discussed in relation to the effectiveness of this modelling approach for post-season modelling and the potential for adapting this approach to assist in-season management. 


\section{Chapter 2: In-season management and post-season assessment modelling}

In-season real time management has the potential to overcome some of the difficulties of managing fisheries for short lived species, such as squid, that tend to live for around one year. For these short-lived species, what can be learnt about the stock in previous years may have little bearing on the current year's stock. Squid tend to have highly variable stock-recruitment relationships and consequently volatile stock levels (Pierce \& Guerra, 1994). Hence, setting catch limits based on what has happened in previous years has the potential to result in under or over exploiting the stock.

While the New Zealand squid fishery is currently managed with a constant TACC, there have been two occasions where the TACC has been extended mid-season. This was in response to high catches and catch rates early in the season. The first was the 2003-04 season which was given a 30\% mid-season increase, which was not reached. The second was the 2005-06 season which was given a $10 \%$ mid-season increase, which was almost obtained (Ministry of Fisheries, 2011). The aim of in-season management would be to better determine whether a season is going to be good enough to increase the TACC, by how much, and with what risks.

Hurst et al. (2012) mentions various assessment methods used for squid stocks in other countries. Those reviewed by Payne et al. (2005) that have been used in Europe included depletion methods (Young et al., 2004) and modified cohort analysis (Royer et al., 2002). Outside Europe, the variety of methods used include: pre-season assessment based on fishing survey data; in-season assessment that uses data from the fishery as the season progresses to estimate the point at which fishing should cease, based on analysis of the influence of cumulative catch (Leslie \& Davis, 1939), or effort (DeLury, 1947), on an abundance index such as CPUE (Catch Per Unit Effort) (Royer et al., 2002), or refinements of these methods (Beddington et al., 1990; Rosenberg et al., 1990; Basson et al., 1996; Agnew., 2002, 2005) and cohort analysis (Royer et al., 2002).

In a preliminary analysis, Hurst et al. (2012) found the CPUE data for the Snares fishery to have the potential to predict season abundance by at least the end of February (the squid fishery occurs between December and May). Length frequency data (described in Chapter 5), may also be modelled with the CPUE indices to predict abundance and escapement with greater confidence than using only the CPUE. The success of this approach requires the CPUE indices to be proportional to biomass and the length frequency data to represent random samples from the catch.

There is some speculation as to whether the CPUE can be assumed to be proportional to biomass. Even if catch and effort data are standardised to remove the impact of all known factors, there is still no guarantee of this (Maunder \& Punt, 2004). For example, many species aggregate strongly (Augustyn \& Roel, 1998) which can lead to potential bias in the index of stock abundance (Campbell, 2004). For the length frequency data to be considered a random sample, the effective sample size needs to be reduced (Chapter 7), reducing the effect data have on the model and the confidence of its results.

Prior to attempting in-season management, it is necessary to first model the length frequency and CPUE data to determine if it is possible to estimate annual escapement rates for a number 
of seasons. Target escapement rates for management purposes commonly aim to ensure at least $40 \%$ of the estimated biomass will survive to spawn (Beddington et al., 1990). 


\section{Chapter 3: The fishery - Snares and Auckland Islands}

The New Zealand squid fishery began in the late 1970's and was introduced to the quota management system on 1 November 1987. The quota management system is used to control the total commercial catch for virtually all the main fish stocks found within New Zealand's 200 nautical mile EEZ (Exclusive Economic Zone) (Ministry of Fisheries, 2011). The New Zealand squid fishery is based on two related species. Nototodarus gouldi is found around mainland New Zealand north of the Subtropical Convergence (Figure 3a), whereas N. sloanii is found in and to the south of the convergence zone (Ministry of Fisheries, 2011).

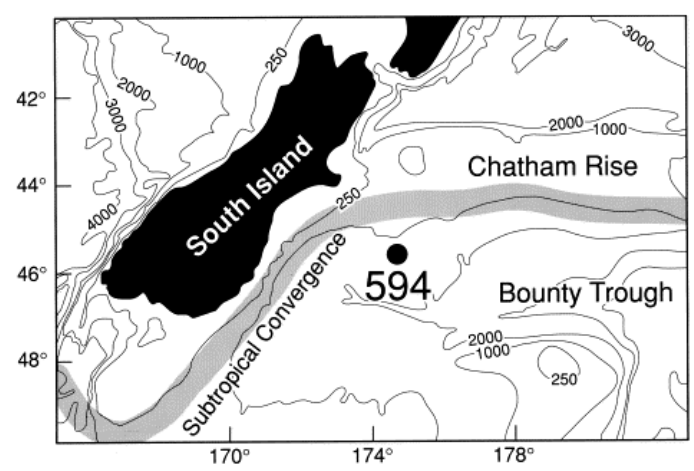

Figure 3a: The position of the Subtropical Convergence (Kowalski \& A. Meyers, 1997).

The New Zealand EEZ is split into 4 Quota Management Areas (QMAs) for the squid fishery (Figure 3b). They are SQU10T (Kermadec Islands), SQU6T (Auckland Islands), and SQU1T \& SQU1J (the rest of the EEZ, further sub-divided by the gear types, trawl and jig).

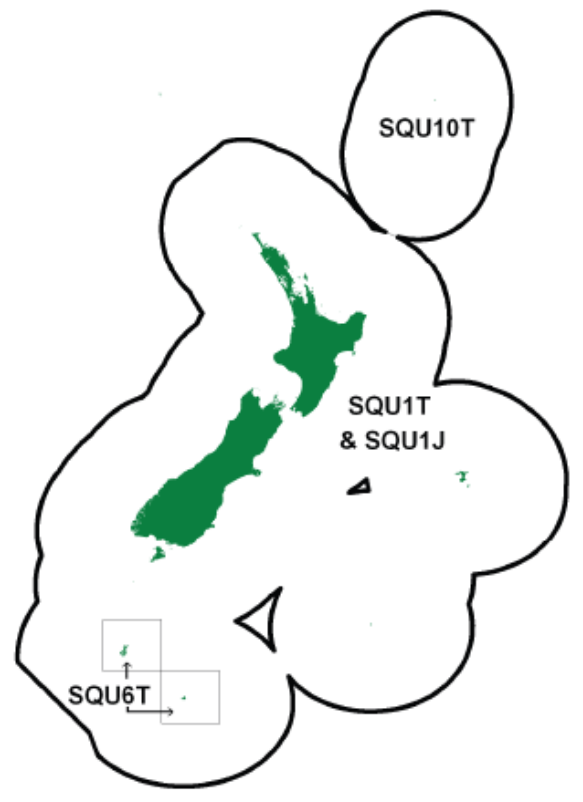

Figure 3b: The NZ EEZ and QMAs (Quota Management Areas) for squid.

The Auckland Islands squid fishery is managed under its own QMA due to the fact it is readily accessible to trawlers and the squid here can be caught with little fin-fish by-catch, making them an attractive resource to trawlers (Ministry of Fisheries, 2011). New Zealand (or Hooker's) sea lions are caught by vessels trawling for arrow squid, most frequently 
around the Auckland Islands (Ministry of Fisheries, 2011). Total landings for the season for Auckland Islands have ranged from approximately 15,000 t to 35,000 t over the last 10 years (Table 3a). The Kermadec Islands squid fishery is negligible, with a Total Allowable Commercial Catch (TACC) of 10 tonnes and 0 reported landings since the management of the squid fishery. Landings from the jig fishery SQU1J have ranged from approximately 800 $\mathrm{t}$ to $9000 \mathrm{t}$ over the last 10 years. The TACC is much higher at 50,212 $\mathrm{t}$.

Of the total catch from SQU1T, 77\% comes from the Snares shelf/Stewart Island (Ministry of Fisheries, 2011). This is an important fishery, known as the 'Snares' fishery (Hurst et al., 2012). Figure 3c shows the Snares and the Auckland Islands boundaries. Total landings for SQU1T have ranged from approximately $16,000 \mathrm{t}$ to 50,000 $\mathrm{t}$ over the last 10 years.

Table 3a: Reported catches $(t)$ and TACCs (t) of arrow squid from 2001-02 to 2009-10 (Ministry of Fisheries, 2011).

\begin{tabular}{lrrrrrrrr} 
Fishing & \multicolumn{1}{c}{ SQU1J } & \multicolumn{3}{c}{ SQU1T } & \multicolumn{2}{c}{ SQU6T } & \multicolumn{2}{c}{ SQU10T } \\
year & Landings & \multicolumn{1}{c}{ TACC } & Landings & TACC & Landings & TACC & Landings & TACC \\
$2001-02$ & 799 & 50,212 & 35,872 & 44,741 & 11,502 & 32,369 & 0 & 10 \\
$2002-03$ & 2,896 & 50,212 & 33,936 & 44,741 & 6,887 & 32,369 & 0 & 10 \\
$2003-04$ & 2,267 & 50,212 & 48,060 & $58,163^{\#}$ & 34,635 & 32,369 & 0 & 10 \\
$2004-05$ & 8,981 & 50,212 & 49,780 & 44,741 & 27,314 & 32,369 & 0 & 10 \\
$2005-06$ & 5,844 & 50,212 & 49,149 & $49,215^{\#}$ & 17,425 & 32,369 & 0 & 10 \\
$2006-07$ & 2,278 & 50,212 & 49,495 & 44,741 & 18,479 & 32,369 & 0 & 10 \\
$2007-08$ & 1,371 & 50,212 & 36,171 & 44,741 & 18,493 & 32,369 & 0 & 10 \\
$2008-09$ & 1,032 & 50,212 & 16,407 & 44,741 & 28,872 & 32,369 & 0 & 10 \\
$2009-10$ & 891 & 50,212 & 16,759 & 44,741 & 14,786 & 32,369 & 0 & 10
\end{tabular}

\# In season increases of 30\% for 2003-04 and 10\% for 2005-06

It is the Snares fishery that this research is focusing on, with the addition of the Auckland Islands for the 2008 fishing year. Previously, these two fisheries have been considered as separate stocks, but it is possible this is not the case. Hurst et al. (2012) found evidence suggesting squid moving between the areas, such as large squid in the Snares disappearing as similar sized squid turned up in the Auckland Islands. The species found in both Auckland Islands and the Snares is N. sloanii.

The squid fishery occurs between December and May, with peak harvest from January-April. The time steps within a season are in weeks, with week 1 corresponding to the first week in December. Fishing years are labelled by their final year where 1990-91 becomes 1991.

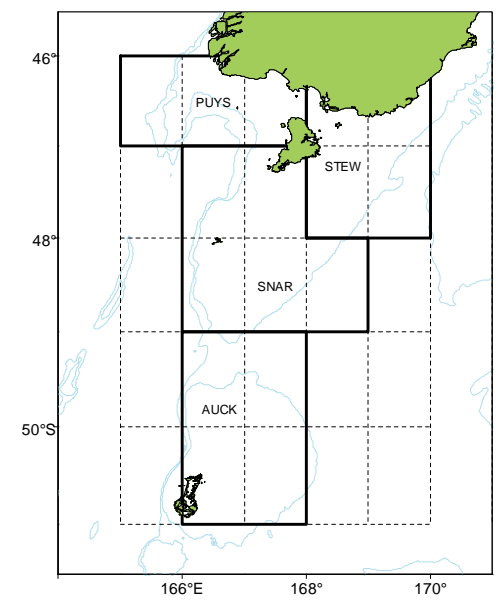

Figure3c: Map showing fishing areas PUYS (Puysegur), STEW (Stewart Islands), SNAR (Snares) and AUCK (Auckland Islands). 


\section{Chapter 4: N. sloanii biology}

This chapter gives background to key aspects of squid biology that affect the model. They are lifespan, spawning, natural mortality, length to weight conversion, abundance estimates and growth in length.

\subsection{Lifespan, spawning and natural mortality}

N. sloanii (or Wellington flying squid) live for around 1 year and growth is rapid. It appears they spawn once and then die. Fish taken by the fishery do not appear to have spawned (Ministry of Fisheries, 2011).

Estimated ages (from daily growth rings in the statolith) suggest $N$. sloanii hatch in July and August, with spawning occurring in June and July (Ministry of Fisheries, 2011). However Uozumi and Ohara (1993) found evidence that suggests spawning occurs throughout the year.

While there are no estimates of natural mortality for $N$. sloanii, there are some for other squid species that live for 1 year, spawn and then die. For the instantaneous natural mortality rate $\mathrm{M}$, these lie in the range $0.007-0.075$ per week. This is a huge range and translates to the probability of surviving one year being anywhere between 0.02 and 0.69 . Such estimates have however sometimes been used in different ways. Royer et al. (2002) split the lifespan of a squid into gnomonic intervals (these increase in duration in proportion to the age up to the start of each interval), the last of which corresponded to the exploited stage (from 6 to 12 months). Constant natural mortality rates of $0.1,0.2$ and 0.3 per month (approximately 0.025, 0.05 and 0.075 per week respectively) were applied to this interval. These give probabilities of surviving the 6 month period of $0.55,0.30$ and 0.17 respectively.

Chen et al. (2008) used values ranging from 0.03 to 0.10 per 10 days (0.021-0.07 per week), with the natural mortality remaining constant over time. (Caddy, 1996) listed several published values for adult pre-spawning squid natural mortality, which were in the range 0.35-1.8 per year (approx. 0.007- 0.035 per week). Roa-Ureta and Arkhipkin (2006) used Hoenig's empirical equation based on longevity (Hewitt and Hoenig, 2005) to arrive at $\mathrm{M} \approx 0.0133 d^{-1}$ (per day) $\left(=0.0931 w^{-1}\right.$ (per week)).

If we postulate a significant increase in natural mortality either immediately after spawning (in the last month, say) or soon after having been spawned (in the first month), then it could be reasonable to expect a low natural mortality for the remaining 10 months of the year. Suppose $50 \%$ are expected to survive these 10 months, then expressing this on a weekly basis gives

$$
P(\text { surviving } 10 \text { months })=e^{-10 \times 4 \times M}=0.5
$$

or

$$
M=-\frac{1}{4 \times 10} \ln (0.5)=0.017 \text { per week }
$$

This is within the range of estimates found in the literature and it seems a reasonable estimate for this project. Hence, $M=0.017 w^{-1}$ is used in the model. 


\subsection{Length to weight conversion}

The length-weight conversion for $N$. sloanii $\in(9 \mathrm{~cm}, 41 \mathrm{~cm})$ DML (Dorsal Mantel Length) for Snares (Hurst et al. 2012) has equation

$$
w=a l^{b}
$$

$w$ is the weight $(\mathrm{g}), a=0.0171, b=3.08$ and $l$ is the length $(\mathrm{cm})$ (Figure 2a).

The length-weight conversion for $N$. sloanii $\in(10 \mathrm{~cm}, 40 \mathrm{~cm})$ DML (Dorsal Mantel Length) for Auckland Islands (Hurst et al. 2012) is

$$
w=a l^{b}
$$

$w$ is the weight $(\mathrm{g}), a=0.0136, b=3.16$ and $l$ is the length $(\mathrm{cm})$ (Figure $2 \mathrm{a})$.

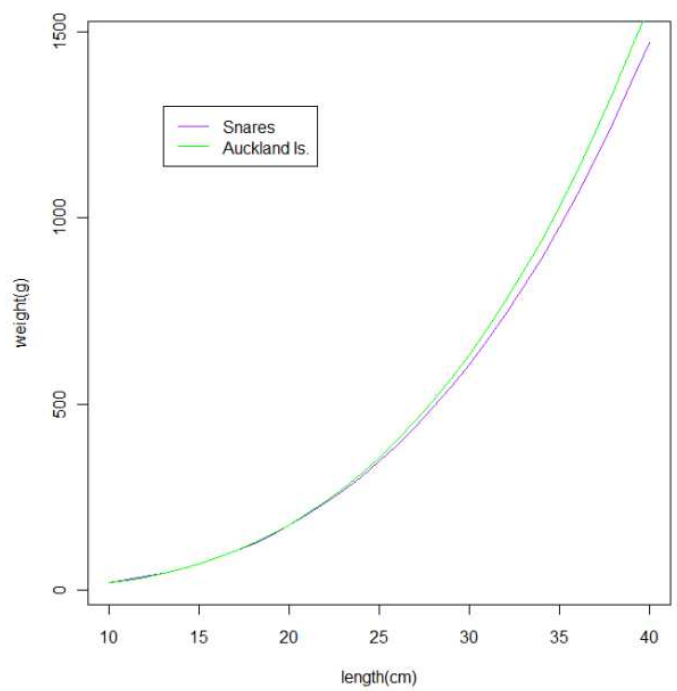

Figure 2a: Length to weight conversion curves for Snares and Auckland Islands

The model will assume that length to weight conversions follow this curve exactly.

\subsection{Abundance estimates}

Early attempts to assess and manage squid stocks in New Zealand initially used an estimated biomass of 600,000 $\mathrm{t}$ for the NZ EEZ, calculated from the areal expansion method based on 1978-79 commercial catch data from both the jig and trawl fisheries (Mattlin \& Coleman, 1988). While this is not considered to be an accurate estimate of stock size (Mattlin \& Coleman, 1988), it seems to be the only attempt at an abundance estimate. It also has limited relevance since the squid are spawning and dying at about one year. One year's abundance may have little bearing on any other year's abundance.

However, in calculating the expected escapement (equation 6.11) it is necessary to calculate the expected population. Hence, having a figure with which to compare the expected population figures calculated here may be helpful.

Since the length frequency data modelled here are in numbers, I have done a (very rough) conversion of $600,000 \mathrm{t}$ to a number as follows. 
The mean length of the squid (taken from the length frequency data) is approximately $25 \mathrm{~cm}$. The weight of a $25 \mathrm{~cm}$ squid (using equation 6.3 and the conversion parameters for the Snares) is

$$
0.0171 \times 25^{3.08} \approx 345 g=0.345 \times 10^{-3} t
$$

This gives approximately

$$
\frac{600,000}{0.345 \times 10^{-3}}=1.7 \times 10^{9}=1,700 \text { million squid }
$$

\subsection{Growth}

\section{Logistic growth parameters}

Assuming logistic growth in length over time,

$$
E[L \mid t]=\frac{L_{\infty}}{\left(1+e^{-K\left(t-t_{0}\right)}\right)}
$$

$E[L \mid t]$ is the expected or average length (cm) at age $t$ (days),

$L_{\infty}$ is the upper asymptote for length $(\mathrm{cm})$,

$K$ is a growth coefficient,

$t_{0}$ is the age in days at which the average length was $\frac{1}{2} L_{\infty}$ and is the age of maximum growth rate.

Uozumi (1998) found the growth rate of $N$. sloanii in New Zealand waters to vary based on gender and hatching month. He estimated logistic growth parameters for each hatching season and gender by examining the relationship between the Dorsal Mantel Length and statolith radius. See Table 2a for Uozumi's parameter estimates.

Table 2a: Estimated growth parameters for $N$. sloanii, by month of hatching (Uozumi 1998).

\begin{tabular}{|lrrr|rrr|}
\hline & & & Male & & & Female \\
\hline Month & $L_{\infty}(\mathrm{cm})$ & $K\left(\right.$ days $\left.^{-1}\right)$ & $t_{0}$ (days) & $L_{\infty}(\mathrm{cm})$ & $K\left(\right.$ days $\left.^{-1}\right)$ & $t_{0}$ (days) \\
\cline { 2 - 7 } January & 42.73 & 0.0212 & 230.0 & 43.85 & 0.0192 & 228.6 \\
February & 33.33 & 0.0267 & 202.4 & 41.97 & 0.0184 & 221.8 \\
March & 31.55 & 0.0242 & 200.1 & 43.48 & 0.0168 & 235.2 \\
April & 32.72 & 0.0205 & 206.6 & 46.87 & 0.0156 & 250.3 \\
May & 33.18 & 0.0204 & 205.3 & 44.47 & 0.0170 & 237.5 \\
June & 32.52 & 0.0219 & 190.4 & 40.78 & 0.0177 & 214.5 \\
July & 34.80 & 0.0196 & 188.8 & 43.42 & 0.0157 & 211.0 \\
August & 40.36 & 0.0167 & 202.0 & 42.09 & 0.0167 & 199.9 \\
September & 38.51 & 0.0194 & 191.4 & 39.35 & 0.0188 & 188.6 \\
October & 34.54 & 0.0223 & 176.1 & 40.08 & 0.0205 & 193.9 \\
November & 28.78 & 0.0313 & 158.9 & 38.38 & 0.0209 & 200.1 \\
December & 60.14 & 0.0123 & 279.7 & 44.62 & 0.0183 & 232.8 \\
\hline
\end{tabular}

For the hatching months that are likely to show in the fishing season (January-April, September-December), I calculated average growth parameters (Table $2 b$ ) with male and female parameters separate and then combined. The logistic growth curves using these parameters are shown in Figure $2 b$. 
Table 2b: Estimated growth parameters for $N$. sloanii, averaged over relevant months for female, male and combined sexes.

\begin{tabular}{|l|rrr|}
\hline & $L_{\infty}(\mathrm{cm})$ & $K$ & $t_{0}($ days $)$ \\
\hline Male & 37.78 & 0.0222 & 205.65 \\
Female & 42.31 & 0.0186 & 218.91 \\
Combined & 40.04 & 0.0204 & 212.28 \\
\hline
\end{tabular}

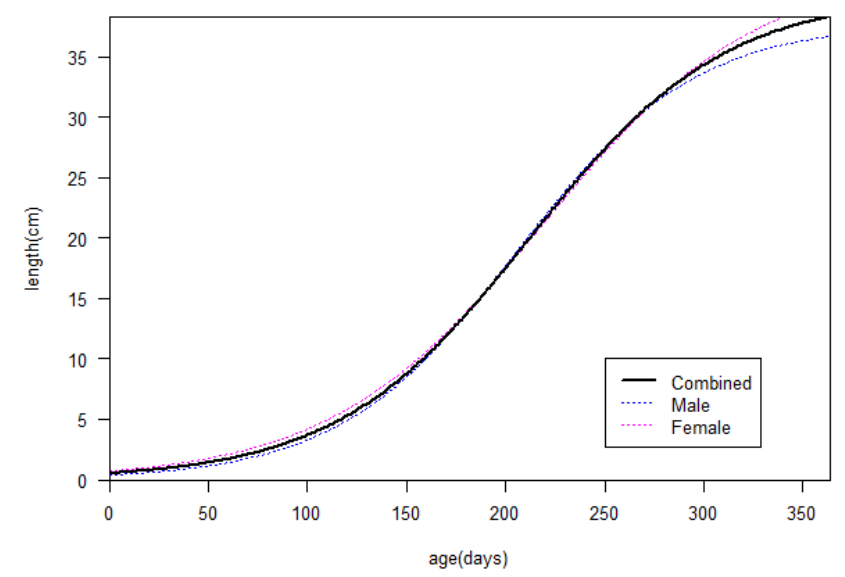

Figure 2b: Expected length vs age for combined sexes, with mean parameters from relevant spawning months.

\section{Expected growth as a function of length}

The instantaneous growth rate is the derivative of the expected length with respect to time, as follows

Let

$$
L(t)=\frac{L_{\infty}}{1+e^{-K\left(t-t_{0}\right)}}
$$

then

$$
\frac{d L}{d t}=K L_{\infty}\left[1+e^{-K\left(t-t_{0}\right)}\right]^{-2} e^{-K\left(t-t_{0}\right)}
$$

which can be written

$$
L K\left[\frac{L_{\infty}-L}{L_{\infty}}\right]
$$

Hence,

$$
\frac{d L}{d t}=L K\left[\frac{L_{\infty}-L}{L_{\infty}}\right]
$$

which is a quadratic in $L$, peaking with maximum growth at $L=\frac{L_{\infty}}{2}$ (Figure 2c). 


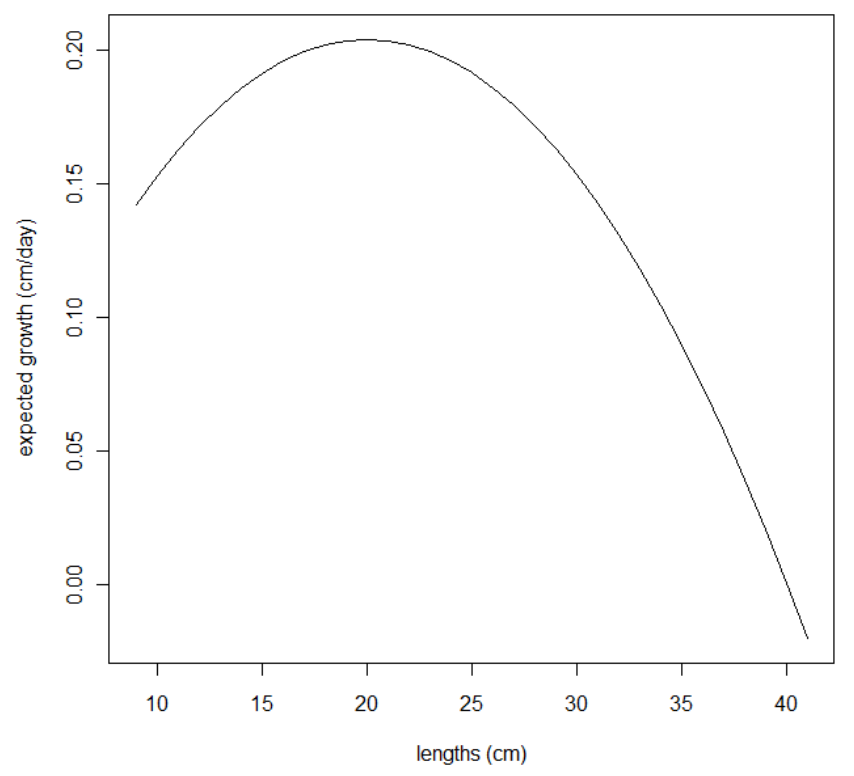

Figure 2c: Instantaneous growth rate using combined average Uozumi parameters (Table 2b).

The change in length in one week (Figure 2d) is calculated as

$$
\begin{gathered}
\Delta L=L(t+7)-L(t) \\
=\frac{L_{\infty}}{1+e^{-K\left(t+7-t_{0}\right)}}-\frac{L_{\infty}}{1+e^{-K\left(t-t_{0}\right)}} \\
=\frac{L_{\infty}}{1+e^{-7 K}\left(\frac{L_{\infty}-L}{L}\right)}-L
\end{gathered}
$$

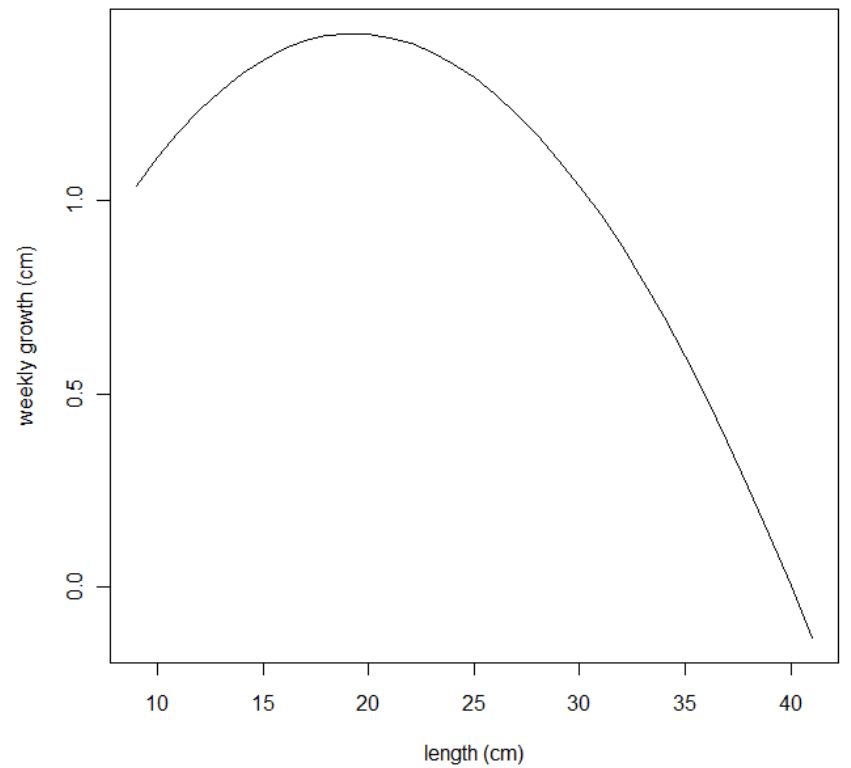

Figure 2d: Weekly growth using combined average Uozumi parameters (Table 2b). 


\section{Expected growth with estimated parameters}

While I do not expect the growth of the squid in the seasons studied in this project to exactly match Uozumi's parameters, it does give insight to the possible shape the mean growth per week is likely to take. Initially, I ran the model estimating a single mean growth parameter, then increased the number of parameters up to 3, testing for improvements in the fit of the model at each increase. Figure $2 d$ shows examples of the mean growth equations with 1,2 , and 3 parameters compared with Uozumi's parameters.

I assumed the same growth rate for males and females. The length frequency plots (Appendix A) show very little difference in the size distributions of male and female squid, suggesting similar growth rates. Mattlin \& Scheilbling (1985) also found the size distributions of male and female $N$. sloanii in New Zealand to be similar for each depth and sampling period.

The single parameter mean growth was assumed to be constant with estimated parameter $g$ such that

$$
[\text { growth in } 7 \text { days } \mid L]=g
$$

The 2 parameter mean growth was assumed to be a linear equation with parameters $m g_{\text {start }}$ and $m g_{\text {final }}$ which are the mean growths at the minimum and maximum lengths respectively, such that

$$
[\text { growth in } 7 \text { days } \mid L]=\text { intercept }+\operatorname{grad} \times\left(L-l_{\min }\right)
$$

$\operatorname{grad}=\frac{g_{\text {final }}-g_{\text {start }}}{l_{\max }-l_{\min }}$

intercept $=g_{\text {start }}-\operatorname{grad} \times l_{\min }$

$l_{\min }(\mathrm{cm})$ and $l_{\max }(\mathrm{cm})$ are the minimum and maximum (respectively) lengths

for which we have length to weight conversion parameters

The 3 parameter mean growth was assumed to be a broken stick linear equation with parameters $g_{\text {final }}, h$ (the length at which the break of the stick occurs (maximum or minimum)) and $g_{h}$ (the mean growth at length $h$ ), such that

$$
[\text { growth in } 7 \text { days } \mid L]= \begin{cases}\operatorname{grad} \times L+g_{h}-\operatorname{grad} \times h, & L<h \\ -\operatorname{grad} \times L+g_{h}+\operatorname{grad} \times h, & L \geq h\end{cases}
$$

$\operatorname{grad}=\frac{g_{h}-m_{\text {final }}}{l_{\max }-h}$ 
$l_{\text {max }}(\mathrm{cm})$ is the maximum length for which we have length to weight conversion parameters This assumes equal slopes with opposite signs for the two segments.

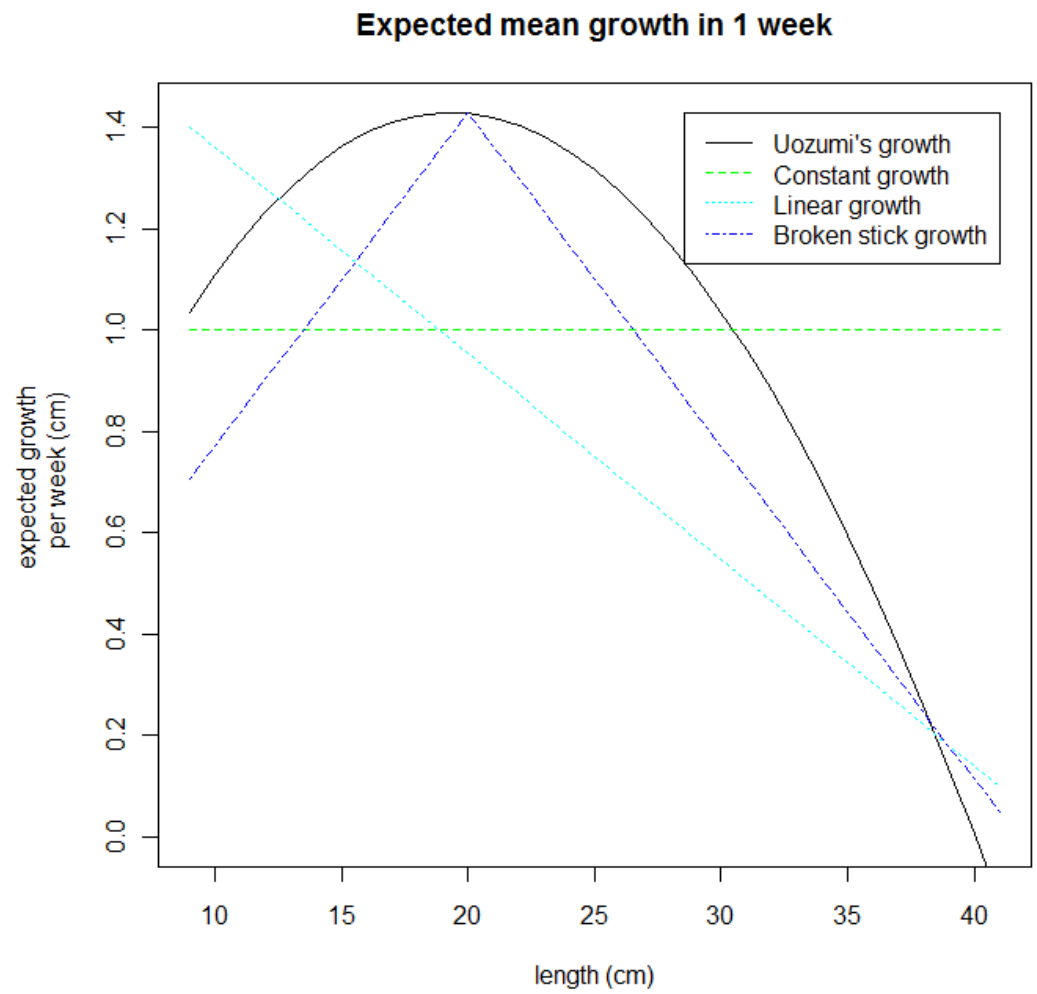

Figure 2d: Examples of expected mean growth using constant, linear and broken stick equations, compared to Uozumi's growth parameters. 


\section{Chapter 5: The data}

The data used in this project are length frequencies from the catch for some weeks, weekly catch weights and CPUE indices for four combinations of area and year: the Snares in 1990, 1991, 2008 and the Auckland Islands in 2008. The data are from the analysis carried out by Hurst et al. (2012) and it is from here that the selection of area and year combinations came. The analyses were also carried out for 1994, but the catch-weight data were inconsistent with the other years (Appendix A) and so this year was dropped from this analysis.

Hurst et al. (2012) explained the length sampling, length frequencies, catch weights and CPUE data as follows.

\section{Length sampling}

The Ministry of Fisheries Observer Programme has collected squid sex and length data for some years since 1986 from mainly the Auckland Islands and Snares fisheries. Weekly length frequency distributions were generated for selected seasons.

The observed catch as a proportion of total target squid catch has ranged from about $2-22 \%$ in the Auckland Islands and about $1-15 \%$ at the Snares. The highest observer squid sampling was achieved in both areas in the 2008 season. Sampling intensity was quite variable with the number of tows sampled per week (Table 5.1) varying from 0 to 61 .

Table 5.1: Number of tows sampled from for each week, for each area and year combination.

\begin{tabular}{|r|rrrr|r|}
\hline week & \multicolumn{3}{|c|}{ Snares } & $\begin{array}{c}\text { Auckland } \\
\text { Islands }\end{array}$ \\
\hline & 1990 & 1991 & 2008 & 2008 \\
\hline 1 & 7 & 0 & 0 & 0 \\
2 & 20 & 0 & 0 & 0 \\
3 & 18 & 0 & 0 & 0 \\
4 & 8 & 11 & 10 & 0 \\
5 & 1 & 28 & 37 & 0 \\
6 & 0 & 61 & 54 & 1 \\
7 & 0 & 42 & 52 & 5 \\
8 & 0 & 36 & 25 & 17 \\
9 & 0 & 24 & 13 & 29 \\
10 & 0 & 41 & 27 & 19 \\
11 & 2 & 3 & 13 & 20 \\
12 & 3 & 32 & 23 & 22 \\
13 & 3 & 23 & 12 & \\
14 & & 15 & 15 & \\
15 & & 7 & 7 & \\
16 & & 0 & 6 & \\
17 & & 0 & 13 & \\
18 & & 2 & 7 & \\
19 & & 2 & 1 & \\
\hline
\end{tabular}

\section{Length frequencies}

Length frequencies were estimated as the weighted average of individual length samples (where the weighting was the estimated catch in tonnes). Length frequency data for each year 
and area were post-stratified by weekly time strata. Length frequencies were determined using the 'catch.at.age' software (Bull \& Dunn 2002) which scales the length frequency from each catch up to the tow catch, sums over catches in each stratum, scales up to the total stratum catch, and then sums across the strata, to yield overall length frequencies. Numbers of squid were estimated from catch weights using the length-weight relationship for observed squid. See Appendix A for numbers-at-length plots.

\section{Weekly catch weights}

The catch weights for each week come from catch and effort data requested from the Ministry of Fisheries catch-effort database "warehou". The data were groomed, with key variable outliers (values outside acceptable bounds for the variable) corrected using median imputation. This involves replacing missing or outlier values with a median value calculated over some subset of the data. See Appendix A for weekly catch weights table.

\section{CPUE (Catch Per Unit Effort)}

CPUE is assumed to be an index of abundance, with abundance assumed proportional to CPUE such that

$$
\text { CPUE }=q \times \text { biomass }
$$

where $q$ is the catchability coefficient.

Weekly CPUE analyses were conducted for Auckland Islands 2008 and Snares 1990, 1991, 1994, 2008 (Appendix A). Estimates of relative week effects in each CPUE model were obtained from a stepwise multiple regression method in which the data were modelled using a lognormal generalised linear model following Dunn (2002). A forward stepwise multipleregression fitting algorithm (Chambers \& Hastie 1991) implemented in the $\mathrm{R}$ statistical programming language ( $\mathrm{R}$ Development Core Team 2003) was used to fit all models. The algorithm generates a final regression model iteratively and uses the week term as the initial or base model in all cases. The reduction in residual deviance relative to the null deviance is calculated for each variable added to the base model. The variable that results in the greatest reduction in residual deviance is added to the base model if this would result in an improvement in the residual deviance of more than $1 \%$. The model repeats this process until no new variables can be added. 


\section{Chapter 6: The model}

The aim of the model is to estimate escapement due to fishing. Escapement is calculated as the population that results from fishing divided by the population that would have existed if no fishing had occurred. In order to estimate these populations, the model uses the length frequency data, CPUE indices and catch weights.

The distributions of the data should be explainable by four processes: new recruitment cohorts that come into the modelled population, squid growth, natural mortality, and fishing mortality. The model attempts to create the distributions using parameters and equations that most closely fit the data. The desired outcomes are to determine if the patterns in the data are sufficient to estimated when the new recruitment cohorts come in, how large they are, what proportion of fish die naturally, what proportion are caught, and which lengths are most likely to be caught. If we can do this, we can then estimate the escapement.

Each season is initially modelled using one recruitment cohort. Further cohorts are added until there is no longer significant improvement as a result.

The initial state of the model has a null population. The population first becomes non-zero when the first recruitment cohort is added to the existing population.

The length distribution of new recruitment cohorts that enter the model follows a normal distribution with estimated parameters (Table 6a). The initial population size in numbers and the timing in weeks for each cohort are also estimated parameters. The timing of each recruitment cohort is a real number, and hence it is likely a new cohort will enter the model mid-week. When this happens, its growth is calculated for the proportion of week that remains until the next whole week. The new cohort is then added to the existing population at the end of this week, such that it takes effect in the model at the beginning of the next week.

Within each time-step (1 week) the sequence of events is: natural mortality is removed, then fishing mortality is removed, then the fish grow (and any new recruits this week grow), then any new recruits are added to the existing population (Figure 6a).

Table 6a: Data and parameters used in the models

\begin{tabular}{|l|l|}
\hline Observed data with error: & \\
\hline $\begin{array}{l}\text { Proportions by length for some weeks } \\
\text { CPUE }\end{array}$ & These are what the model is fit to \\
\hline Assumed known exactly: & Used to calculate the exploitation rate \\
\hline Catch weights per week & $\begin{array}{l}\text { Dorsal mantel length (DML) bins for } \\
\text { which there are length-weight } \\
\text { conversion parameters }\end{array}$ \\
\hline Length bins & $\begin{array}{l}\text { Defined for DML 9-41cm } \\
\text { Defined for DML 9-41cm }\end{array}$ \\
\hline $\begin{array}{l}a_{\text {snar }} \\
b_{\text {snar }}\end{array}$ &
\end{tabular}




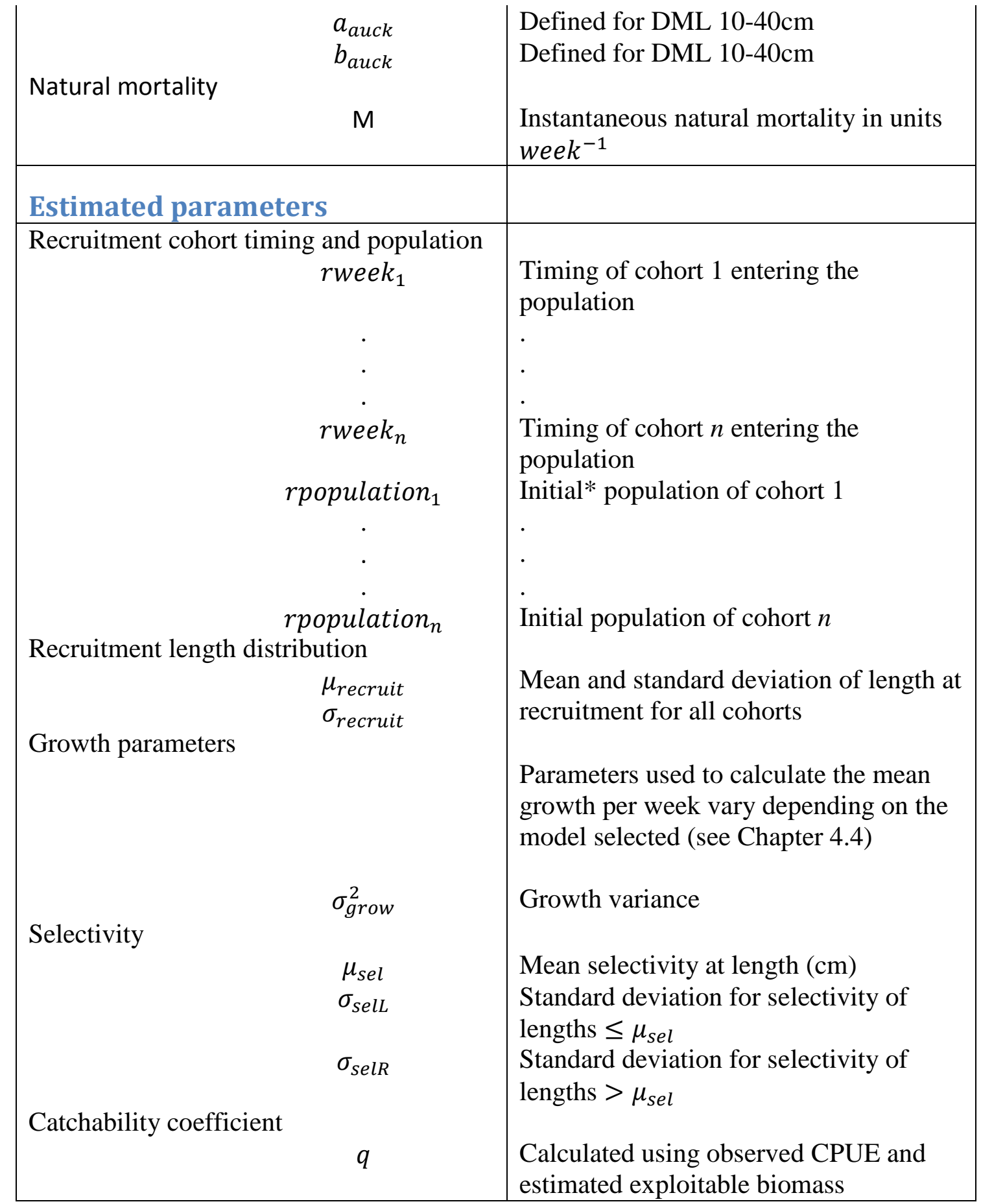

*at time rwee $_{n}$ 
Figure 6a: Procedure for the model

For weeks $w=($ start week, $\ldots$, first fishing week, $\ldots$, last fishing week $)$

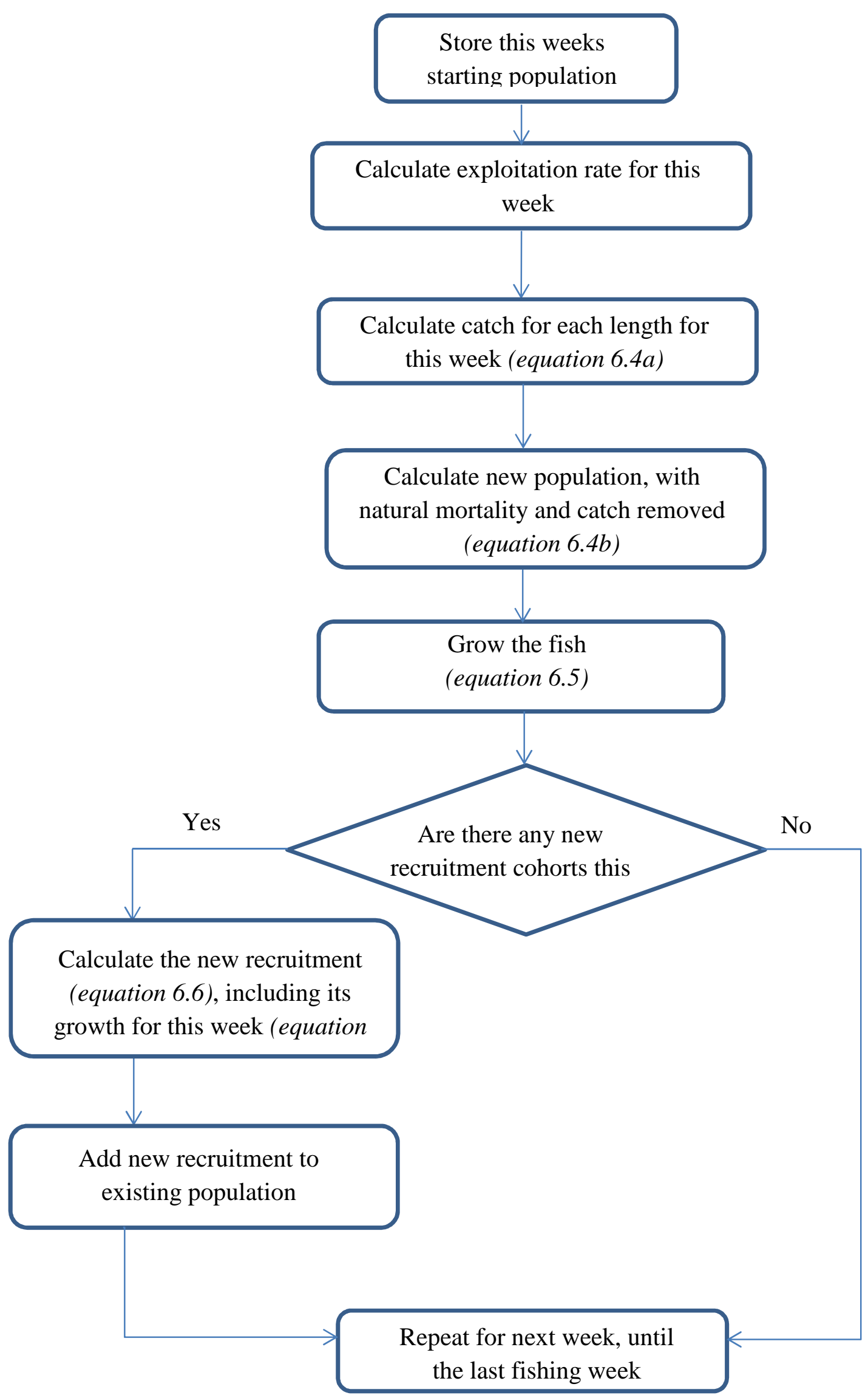




\section{Equations}

These equations are programmed in R code and ADMB code (Appendix C).

\section{Equation 6.1: Selectivity}

$l=$ length

$$
S_{l}= \begin{cases}2^{-\left(\left(l-\mu_{s e l}\right) / \sigma_{L}\right)^{2}}, & l \leq \mu_{\text {sel }} \\ 2^{-\left(\left(l-\mu_{s e l}\right) / \sigma_{R}\right)^{2}}, & l>\mu_{\text {sel }}\end{cases}
$$

$l=$ length $(\mathrm{cm})$

$\mu_{\text {sel }}=$ mean length at selectivity $(\mathrm{cm})$

$\sigma_{\text {selL }}^{2}, \sigma_{\text {selR }}^{2}=$ selectivity variance (left and right respectively)

$S_{l}=$ selectivity for length $l$

\section{Equation 6.2: Exploitation}

$$
U_{w}=\frac{W_{w}}{B_{\text {exp }, w}}=\frac{W_{w}}{\sum_{l}\left(N_{l, w} e^{-M} S_{l} a l^{b}\right)}
$$

$U_{w}$ is the exploitation rate for week $w$

$a, b$ are length $\rightarrow$ weight conversion parameters

$S_{l}$ is the selectivity for length $l$ (Equation 6.1)

$W_{w}$ is the catch weight for week $w$

$N_{l, w}$ is the population in numbers for length $l$, week $w$

$M$ is the natural mortality

$B_{\text {exp,w }}$ is the exploitable biomass in week $w$

\section{Equation 6.3: Length to weight conversion}

Parameters $a$ and $b$ are taken from Hurst et al. (2012) and are stated here in Chapter 4.3

$$
w=a l^{b}
$$

$w$ is the weight $(g)$

$l$ is the length $(\mathrm{cm})$

\section{Equation 6.4a: Catch}

$$
C_{l, w}=N_{l, w} e^{-M} S_{l} U_{w}
$$

$C_{l, w}$ is the catch in numbers for length l and week $w$

$N_{l, w}$ is the population in numbers for length l and week $w$ before mortality 
$M=$ instantaneous natural mortality

$S_{l}=$ selectivity for length $l$ (Equation 6.1)

$U_{w}=$ exploitation rate for week $w$ (Equation 6.2)

\section{Equation 6.4b: Population after catch}

$$
N_{l, w}^{\prime}=N_{l, w} e^{-M}-C_{l, w}
$$

$N^{\prime}{ }_{l, w}$ is the population for length $l$, week $w$, after natural and fishing mortality have been taken out

\section{Equation 6.5a: Growth}

The probability of going from one length to another is

$$
G\left(l_{1}, l_{2} \mid p\right)=\left\{\begin{array}{cc}
\frac{\exp \left(-\frac{\left(l_{2}-l_{1}-p \mu_{g, l_{1}}\right)^{2}}{2 p^{2} \sigma_{\text {grow }}^{2}}\right)}{\sum_{k=l_{1}}^{n l} \exp \left(-\frac{\left(k-l_{1}-p \mu_{g, l_{1}}\right)^{2}}{2 p^{2} \sigma_{\text {grow }}^{2}}\right)}, & l_{2} \geq l_{1} \\
0, & \text { otherwise }
\end{array}\right.
$$

$l_{1}$ is the current length $(\mathrm{cm})$

$l_{2}$ is the future length $(\mathrm{cm})$

$p$ is the proportion of time step (default is 1 ), $p \in[0,1]$

The denominator ensures the probability of going from one length to any other sums to 1 . Thus, no fish are lost or gained in the growing process.

\section{Equation 6.5b Mean growth}

1 parameter

$$
\mu_{g, l}=g
$$

2 parameters

$$
\mu_{g, l}=\text { intercept }+\operatorname{grad} \times\left(l-l_{\min }\right)
$$

$\operatorname{grad}=\frac{g_{\text {final }}-g_{\text {start }}}{l_{\max }-l_{\min }}$

intercept $=g_{\text {start }}-$ grad $\times l_{\min }$ 


$$
\mu_{g, l}=[\operatorname{growth} \text { in } 7 \text { days } \mid L]= \begin{cases}\operatorname{grad} \times L+g_{h}-\operatorname{grad} \times h, & L<h \\ -\operatorname{grad} \times L+g_{h}+\operatorname{grad} \times h, & L \geq h\end{cases}
$$

$\operatorname{grad}=\frac{g_{h}-g_{\text {final }}}{\text { lengths } s_{\max }-h}$

Equation 6.6: Length distribution at recruitment

$$
R_{l}=R_{\text {pop }}\left(\frac{\exp \left(\frac{-\left(l-\mu_{\text {recruit }}\right)^{2}}{2 \sigma_{\text {recruit }}^{2}}\right)}{\sum_{l}\left[\exp \left(\frac{-\left(l-\mu_{\text {recruit }}\right)^{2}}{2 \sigma_{\text {recruit }}^{2}}\right)\right]}\right)
$$

l is the length

$w$ is the current week

$R_{l}$ is the number of recruits at length $l$

$R_{\text {pop }}$ is the number of recruits

$\mu_{\text {recruit }}$ is the new recruitment mean length

$\sigma_{\text {recruit }}$ is the standard deviation for the new recruitment length

\section{Equation 6.7: Natural mortality}

Probability of a fish surviving natural mortality for 1 week $=e^{-M}$

Equation 6.8: Likelihood for proportions at length (Fournier et al., 1990)

This is a robustified variation of the multinomial likelihood

$$
-\log (L)=0.5 \sum_{w} \sum_{l}\left[\log \left(E_{l, w}^{\prime}\right)\right]-\sum_{w} \sum_{l}\left[\log \left[\exp \left(\frac{-\left(O_{l, w}-E_{l, w}\right)^{2}}{2 E_{l, w}^{\prime} / n_{w}^{\prime}}\right)+0.01\right]\right]
$$

where $E_{l, w}^{\prime}=\left(1-E_{l, w}\right) E_{l, w}+\frac{0.1}{n f w \times n l}$

$E_{l, w}=\frac{N_{l, w}}{\sum_{l} N_{l, w}}$

$n f w$ is the number of fishing weeks for which we have length frequency data nl is the number of length bins

$O_{l, w}$ is the observed proportion for length $l$, week $w$ 
$n_{w}^{\prime}$ is the number of tows sampled in week $w$

Equation 6.9: CPUE likelihood (Bull et al., 2008)

The error in the observed CPUE is assumed lognormally distributed with covariance $c$

$$
-\log (L)=\sum_{w}\left[\log (\sigma)+0.5\left(\frac{\log \left(C_{w} / q X_{w}\right)}{\sigma}+0.5 \sigma\right)^{2}\right]
$$

where $\sigma=\sqrt{\log \left(1+c^{2}\right)}$ and $c$ is assumed $0.3 \forall w$

$C_{w}$ is the observed CPUE in week $w$

$X_{w}$ is the estimated exploitable biomass in week $w$

$q$ is the catchability coef ficient

Equation 6.10: Calculating q as a nuisance parameter (Bull et al., 2008)

$$
q=\exp \left(\frac{0.5 n f w+\sum_{w}\left[\log \left(C_{w} / X_{w}\right) / \sigma^{2}\right]}{\sum_{w}\left[1 / \sigma^{2}\right]}\right)
$$

where $C_{w}, X_{w}$ and $\sigma$ are as in Equation 6.9

Equation 6.11: Objective function

$$
f=F_{L}+C P U E_{L}+c w_{\text {penalty }}
$$

where $F_{L}$ is the Fournier log likelihood (Equation 6.8)

CPUE $E_{L}$ is the CPUE log likelihood (Equation 6.9)

$c w_{\text {penalty }}$ is a penalty term applied when the expected catch weight differs from the actual catch weight and is set at 1000

\section{Equation 6.12: Escapement}

This is a proportion, calculated as the population that is estimated to exist after fishing has occurred, divided by the population would have existed with no fishing.

$$
\text { depletion }=\frac{(\text { Number of fish } \mid \text { fishing })}{(\text { Number of fish } \mid \text { no fishing })}
$$

\section{Equation 6.13: Variance of standardised difference of mean lengths}

A measure of variance that takes into account the sample sizes for each week (See Chapter 7 for further details).

$$
\omega=\operatorname{Var}\left[\left(\bar{O}_{w}-\bar{E}_{w}\right) / \sqrt{\left(v_{w} / \widetilde{N}_{w}\right)}\right]
$$


where $v_{w}=\sum_{l=1}^{n l}\left(l^{2} E_{l, w}\right)-\bar{E}_{w}^{2}$

$\bar{O}_{w}$ and $\bar{E}_{w}$ are the observed and expected mean lengths in week $t$ (respectively)

$\widetilde{N_{w}}$ is the number of tows sampled from in week $w$ 


\section{Chapter 7: Finding an appropriate multinomial sample size}

It is assumed that the length frequency data is a simple random sample; a sample of $n$ units out of the $N$ population units such that approximately every one of the ${ }_{N} C_{n}$ distinct samples has an equal chance of being drawn (Cochran, 1977). If for each week, the $n$ squid measured is considered to be the sample of $n$ units, then it is not a simple random sample. Every such sample comes from measuring some squid, from some tows, from some trips by some boats. Hence, every possible sample of $n$ squid does not have an equal chance of selection. It is commonly observed that fish in the same tow tend to be more similar to each other in length than are fish from different tows (Francis, 2011). Hence, in order to avoid over weighting these it is necessary to use a sample size that is much less than the number of fish that have been measured. Reducing the sample size down to the number of tows (Table 5.1) from which squid were measured, results in a closer approximation to the correct weighting for a simple random sample.

There are two ways to test the appropriateness of the sample sizes in relation to the data and simple random sample requirement. One is visually, using plots of the mean lengths. The other is to calculate the variance of the standardised error of mean lengths.

\section{1: Testing the sample size visually}

One way to visualise what the appropriate sample sizes ought to be is to plot the mean lengths for each time step, including the $95 \%$ confidence interval for each mean (equation 7.1.1) using the assumed sample sizes. There should be no jumps in the mean lengths that are not covered by the confidence interval which cannot be explained by the model (such as new recruitments coming in which could lower the mean length significantly in one time-step). If there are such jumps, the estimated sample size is too high. Figures 7.1a and 7.1b demonstrate this technique. Both use a constant sample size for all weeks, but the idea is still the same. The first plot (Figure 7.1a) is an example where the sample size has been set too high. The model is unlikely to be able to fit the jump in mean length from week 10 to week 11. With the confidence intervals, this would require an increase of nearly $3 \mathrm{~cm}$ in one week which is too high to be explained by growth or any other element of the model. There is another jump from week 13 to week 14 which may also be difficult to fit given the fairly gradual slope of the rest of the data points, which suggest slower growth. The second plot (Figure 7.1b) shows the same data but with a smaller sample size and hence larger confidence intervals. The jump in mean length from week 10 to week 11 is now much smaller relative to the confidence intervals. The confidence intervals of weeks 13 and 14 now overlap, requiring no increase of mean length from the model for this week.

Equation 7.1.1: 95\% confidence interval

$$
95 \% C I_{w}=\bar{x}_{t} \pm 1.96 \frac{s_{t}}{\sqrt{N_{w}}}
$$

$N_{w}$ is the assumed multinomial sample size for week $w$

$\bar{x}_{w}$ is the sample mean length at week $w$ (equation 7.1.2)

$s_{w}^{2}$ is the sample variance of the mean length at week $w$ (equation7.1.3) 


$$
\bar{x}_{w}=\sum_{l}\left(O_{l, w} l\right)
$$

$\bar{x}_{w}$ is the sample mean length at week $w$

$l$ is the length bin $(\mathrm{cm})$

$O_{l, w}$ is the observed proportion for length l at week $w$

Equation 7.1.3: sample variance of mean length

$$
s_{w}^{2}=\sum_{l}\left[O_{l, w}\left(l-\bar{x}_{w}\right)^{2}\right]
$$

$s_{w}^{2}$ is the sample variance for week $w$

(a)

(b)
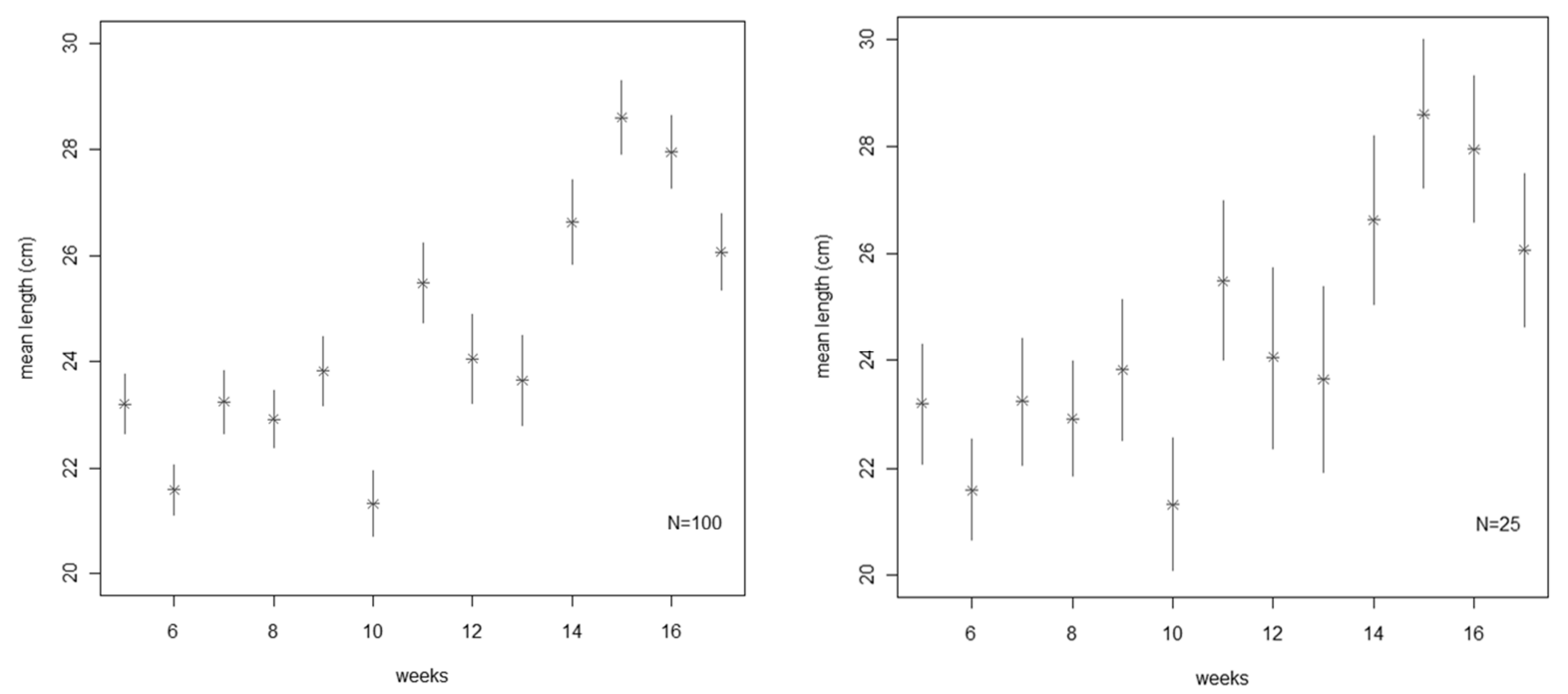

Figure 7.1: Observed mean length $(*)$. The vertical lines are $95 \%$ confidence intervals, calculated assuming simple random sampling: a) $\mathrm{N}=100, b) \mathrm{N}=25$. Plots produced for the purpose of illustration only.

\section{2: Calculating the variance of the standardised error of mean lengths}

The idea for this method comes from the use of a weighting parameter to calculate a new sample size that is more appropriate for the data. This weighting method is explained by Francis (2011). Instead of using it to calculate a new sample size, I will use it to check the current sample sizes (these being the number of tows for each week). This is because these sample sizes are already very low and while I do not want to use them with blind faith, lowering them even further may lead to gaining very little information from the data. In the Fournier Likelihood (Equation 6.8), a very low sample size results in the part of the sum containing the observations tending to zero, thus reducing the effect these data have on the model. 
The aim is to standardise the residuals $\left(\bar{O}_{w}-\bar{E}_{w}\right)$ so that they all have the same variance and then see whether this variance is consistent with the assumed sample sizes. $\bar{O}_{w}$ and $\bar{E}_{w}$ are the observed and estimated mean lengths for week $w$.

The parameters $X_{l, w}$, are estimated using the constraint that the standardised errors

$$
S_{l, w}=\frac{\left(\bar{O}_{w}-\bar{E}_{w}\right)}{X_{l, w}}
$$

have constant variance $=1$

$$
\operatorname{Var}\left(S_{l, w}\right)=1, \forall l, w
$$

If our data set is a simple random sample, then

$$
\operatorname{Var}\left(\bar{O}_{w}-\bar{E}_{w}\right)=\frac{v_{w}}{N_{w}}
$$

where

$$
v_{w}=\sum_{l}\left(l^{2} E_{l, w}\right)-\bar{E}_{w}^{2}
$$

is the variance of the expected length distribution.

Letting $X_{l, w}=\sqrt{\frac{v_{w}}{\widetilde{N_{w}}}}$ where $\widetilde{N}_{w}$ is the number of tows for week $w$,

$$
\begin{aligned}
\operatorname{Var}\left(S_{l, w}\right) & =\operatorname{Var}\left(\frac{\left(\bar{O}_{w}-\bar{E}_{w}\right)}{X_{l, t}}\right) \\
& =\left(\frac{1}{X_{l, w}}\right)^{2} \operatorname{Var}\left(\bar{O}_{w}-\bar{E}_{w}\right) \\
& =\left(\left(\frac{v_{w}}{\widetilde{N}_{w}}\right)^{-0.5}\right)^{2} \frac{v_{w}}{N_{w}} \\
& =1 \text { iff } \widetilde{N_{w}}=N_{w} \forall w
\end{aligned}
$$

$\operatorname{Var}\left(S_{l, w}\right)$ is used to test whether the numbers of tows per week represent appropriate sample sizes. How close it is to 1 will indicate how much the data are being under or over weighted. Values much less than 1 suggest underweighting of the data and values much greater than 1 suggest overweighting of the data. 


\section{Chapter 8: Fitting the data}

\section{1: How many cohorts and what shape function for mean growth?}

How many recruitment cohorts there are and what shape the mean growth takes are two major questions affecting the model chosen to best fit the data. The number of cohorts required is generally easier to determine. It can be seen by analysing the length frequency plots and mean length plots. In the length frequency plots, a bi-modal curve will be indicative of a second cohort, a tri-modal curve indicating a third cohort and so on. In the mean length plots, a sudden decrease in mean length, followed by growth similar to that before the decrease, suggests a new cohort. It is sometimes easier to see new cohorts in the mean length plot than the length frequency plot. It is possible for a new cohort to spread out the peak on a length frequency plot and not show as an extra peak. In this case, the mean length will still drop and this will be easier to pick out than a flattening of the length frequency curve. Figure 8.1a is an example of a new cohort that does not produce a bi-modal curve in the catch, even though the catch is taken as a proportion from the selected population.

Once the number of cohorts has been estimated, the shape the mean growth takes can be decided on the basis of an improvement in the fit to the data as the complexity of the mean growth function increases. If there is no significant improvement to the fit as a result of using a more complex mean growth function, then the simpler model will suffice and hence be chosen. The functions for mean growth that I am testing for are covered in section 4.4.

The objective function (Equation 6.11) and the variance of the standardised error of mean lengths (Equation 6.13) can be used to help determine whether significant improvement has resulted from a model using more cohorts and/or more complex mean growth. The objective function will not be used formally (such as in Akaike's Information Criterion) as this has often been found to be unreliable with fisheries models. It will only be used to assist in the analysis of the mean length, length frequency and CPUE plots. The objective function is always less for a model that is a better fit. The variance of the standardised error of mean lengths is usually less, but can be greater. It is possible for the mean lengths to differ more for a model that is a better fit. For example, when there are two peaks in the length frequency data, the mean length sits somewhere between the peaks. Hence, a model that fits both peaks may fit the mean length worse than a model that fits the length frequencies poorly with only one peak, but fits the mean length very well.

The selectivity curve defines the part of the population that the catch is taken from. It has a significant effect on the parameters chosen to fit the model as it is only the selected (i.e. vulnerable) population that is fitted to the data. The example plot of a population and the selected population (Figure 8.1a) only the population around the selectivity mean length make up the selected population. Those shorter than this will show up in later weeks, but those longer have very little effect on how the model fits to the data. This is worth bearing in mind when examining the growth curves selected, as in some cases they may be unrealistic for squid of larger lengths, which is less of a concern given the selectivity.

Following is a full analysis of the models fitted to the Snares 2008 data. For the remaining datasets, I have presented only a summary. All plots and tables relating to the analyses are included in Appendix B. 


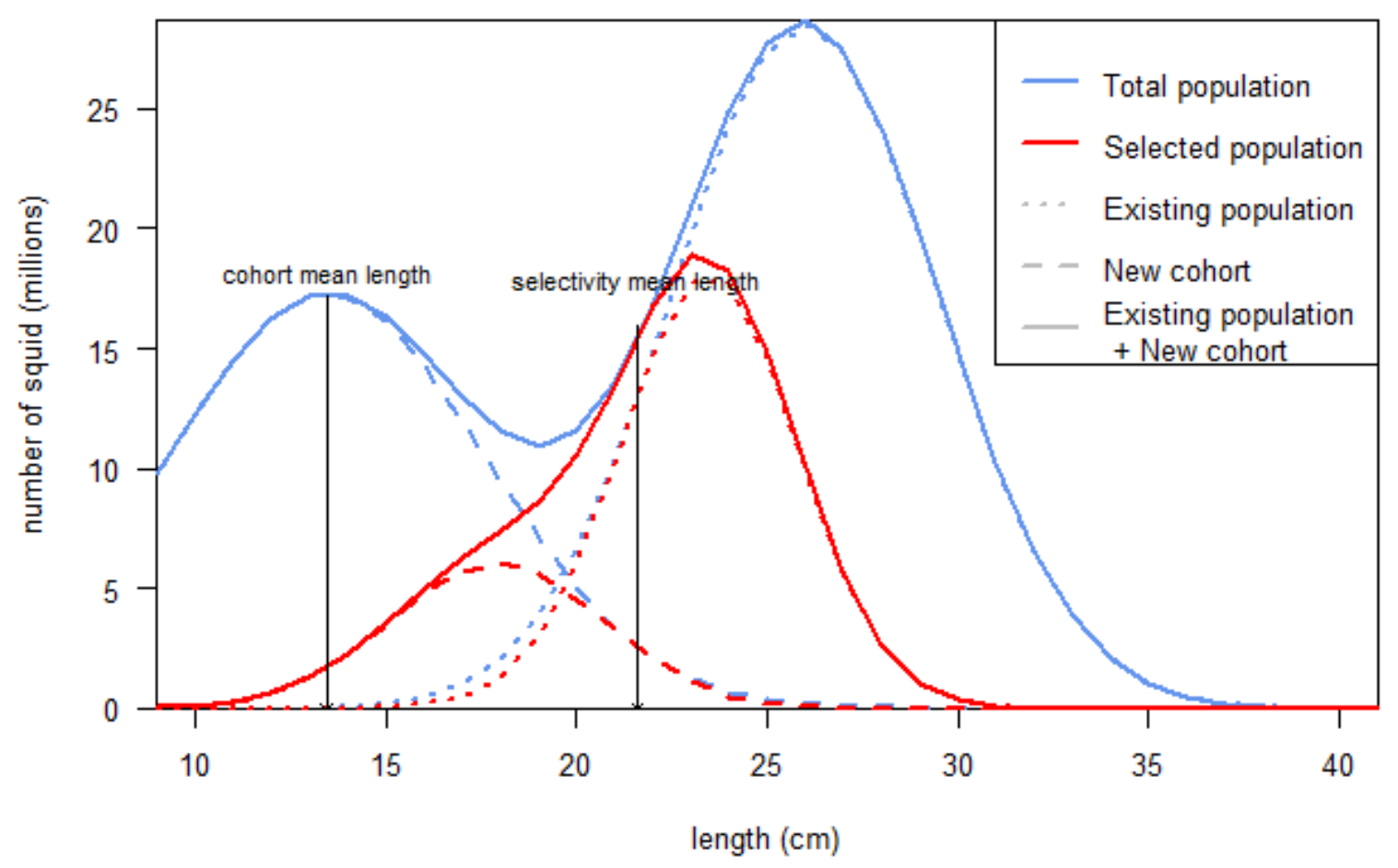

Figure 8.1a: Schematic showing a new recruitment cohort coming into the total population and the selected (vulnerable part of the) population from which the catch is taken. The colour of the line refers to the total or selected population. The type of line indicates the existing population, the new recruitment and the resulting population from adding the new recruitment to the existing population.

\section{Snares 2008}

A 1-cohort model was not sufficient to fit the Snares 2008 data. The mean length plot (Figure 8.1.1c) shows a drop in mean length between weeks 4 and 5, which a model with only 1cohort cannot fit. This new cohort is also evident in the length frequency plot (Figure 8.1.1d) which shows bi-modality beginning at week 5 and merging to a single mode over the next few weeks. Interestingly, in the models with linear and broken stick growth functions, the 1cohort model manages to fit this bi-modal curve by using the recruitment mean length to replicate one peak and the selectivity mean length to replicate the other. However, in doing this the bi-modal curve also exists at week 4 which is not the case in the data. Had the bimodality been present for the first week, these models would be able to fit the data with only 1 -cohort even though it would look like two were being used.

Increasing the number of cohorts from two to three gives little improvement in the fit. It is difficult to see any improvement in the length frequency (Figure 8.1.1d) and mean length fits (8.1.1c). The CPUE plots (Figure 8.1.1b) show some improvement, the most evident being in the bottom right panel which compares 1,2 and 3-cohorts with linear growth. The curve goes slightly higher into the peak around week 10 with the 3-cohort model. However, it is not enough of an improvement to warrant the additional two parameters of a third cohort.

For the 1-cohort model, constant growth is inadequate to fit the data, while for the 2-cohort and 3-cohort models constant growth fits almost as well as linear or broken stick growth or 3cohort models in Figure 8.1.1b. The mean growth plot (Figure 8.1.1c) for 2-cohorts shows the only improvement to be a small amount at week 5 . This is almost impossible to see on the 
length frequency plots (Figure 8.1.1d). The CPUE pots (Figure 8.1.1b) show a bit more of an improvement. However, given none of the models fit terribly well to the CPUE, this improvement is not significant enough. The objective function improves slightly, but not substantially.

For the 1-cohort model using the linear and broken stick growth equations, growth increased continuously with length (Figure 8.1.1a). This is unrealistic and gives further evidence of the inadequacies of the 1-cohort model. The 2-cohort and 3-cohort models with linear growth exhibit similar growth with that of the 3-cohort model slightly slower.

The variance of the standardised error of mean lengths for the 2-cohort linear growth model is 0.81 (Table 8.1.1a). As this is less than 1, it is likely the length data were not overweighted.

\section{Ideal number of cohorts: 2}

Ideal mean growth function: Constant (1 parameter)

Table 8.1.1a: Calculated objective function and variance of standardised error of mean lengths for model fitted with 1, 2, and 3-cohorts and constant, linear and broken stick mean growth for Snares 2008.

\begin{tabular}{|c|c|c|c|c|c|c|}
\hline & \multicolumn{3}{|c|}{ Objective function } & \multicolumn{3}{|c|}{$\begin{array}{l}\text { Variance of standardised } \\
\text { error of mean lengths, } \\
\operatorname{Var}\left(S_{l, w}\right)\end{array}$} \\
\hline Mean growth & constant & linear & $\begin{array}{c}\text { broken } \\
\text { stick }\end{array}$ & $\begin{array}{c}\text { constan } \\
\mathrm{t}\end{array}$ & linear & $\begin{array}{c}\text { broken } \\
\text { stick }\end{array}$ \\
\hline $\begin{array}{l}\text { Number of } \\
\text { cohorts }\end{array}$ & & & & & & \\
\hline 1 & -1042.86 & $\begin{array}{l}- \\
1058.83\end{array}$ & -1059.25 & 1.58 & 1.21 & 1.17 \\
\hline 2 & -1067.92 & $\begin{array}{l}- \\
1070.16\end{array}$ & -1070.17 & 0.81 & 1.03 & 1.02 \\
\hline 3 & -1068.87 & $\begin{array}{l}- \\
1070.50\end{array}$ & -1073.35 & 0.92 & 1.06 & 0.89 \\
\hline
\end{tabular}

Table 8.1.1b: Timing and size of cohorts for models fitted with 1, 2, and 3-cohorts and constant, linear and broken stick mean growth for Snares 2008.

\begin{tabular}{|l|cr|r|}
\hline 1-cohort & \multicolumn{2}{|c|}{ Cohort 1 } & \multirow{2}{*}{ Total size } \\
\cline { 1 - 2 } Mean growth & $\begin{array}{c}\text { wee } \\
\mathrm{k}\end{array}$ & \multicolumn{1}{c|}{ size* } & \\
\hline constant & 0.9 & 213 & 213 \\
linear & 1.4 & 2,741 & 2,741 \\
Broken stick & 1.3 & 2,615 & 2,615 \\
\hline
\end{tabular}

\begin{tabular}{|l|cr|rr|r|}
\hline 2-cohorts & \multicolumn{2}{|c|}{ Cohort 1 } & \multicolumn{2}{c|}{ Cohort 2 } & \multirow{2}{*}{ Total size } \\
\cline { 1 - 4 } Mean growth & wee & size* & week & size* & \\
& $\mathrm{k}$ & & & & \\
\hline constant & -8.0 & 2,741 & 4.3 & 1,957 & 4,698 \\
linear & -7.8 & 2,466 & 4.9 & 1,889 & 4,355 \\
Broken stick & -7.8 & 2,729 & 5.0 & 2,056 & 4,785 \\
\hline
\end{tabular}

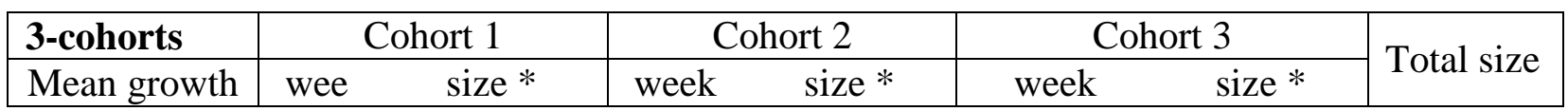




\begin{tabular}{|l|rr|rr|rr|r|}
\hline & $\mathrm{k}$ & & & & & \\
\hline constant & -8.3 & 2,465 & 4.3 & 975 & 5.0 & 655 & 4,095 \\
linear & -6.6 & 2,248 & 4.1 & 1,537 & 7.0 & 508 & 4.293 \\
Broken stick & -5.0 & 2,741 & 4.9 & 1,599 & 5.2 & 1,124 & 5,464 \\
\hline
\end{tabular}

*numbers in millions

Table 8.1.1c: Estimated parameters for Snares 2008 models with 1, 2, and 3-cohorts and constant, linear and broken stick mean growth for Snares 2008.

\begin{tabular}{|c|c|c|c|c|c|c|c|}
\hline $\begin{array}{l}\text { Number of } \\
\text { cohorts }\end{array}$ & $\begin{array}{l}\text { Mean } \\
\text { growth }\end{array}$ & $\begin{array}{l}\mu_{\text {sel }} \\
(\mathrm{cm})\end{array}$ & $\sigma_{\text {SelL }}$ & $\sigma_{\text {SelR }}$ & $\begin{array}{l}\mu_{\text {recruit }} \\
\text { (cm) }\end{array}$ & $\sigma_{\text {recruit }}$ & $\begin{array}{l}\text { Escapem } \\
\text { ent }\end{array}$ \\
\hline \multirow{3}{*}{ 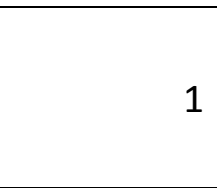 } & constant & 25.02 & 6.62 & 2.80 & 9.07 & 8.59 & 0.85 \\
\hline & linear & 22.00 & 4.97 & 3.61 & 18.75 & 3.60 & 0.95 \\
\hline & broken stick & 22.30 & 5.16 & 3.46 & 13.37 & 6.63 & 0.94 \\
\hline \multirow{3}{*}{2} & constant & 22.00 & 5.61 & 3.60 & 15.82 & 2.96 & 0.89 \\
\hline & linear & 21.62 & 4.46 & 3.58 & 13.45 & 3.53 & 0.89 \\
\hline & broken stick & 21.61 & 4.47 & 3.58 & 13.47 & 3.53 & 0.90 \\
\hline \multirow{3}{*}{3} & constant & 21.91 & 5.80 & 3.59 & 16.52 & 3.08 & 0.88 \\
\hline & linear & 21.57 & 4.68 & 3.57 & 13.16 & 3.64 & 0.90 \\
\hline & broken stick & 21.46 & 5.06 & 3.76 & 17.91 & 2.58 & 0.92 \\
\hline
\end{tabular}

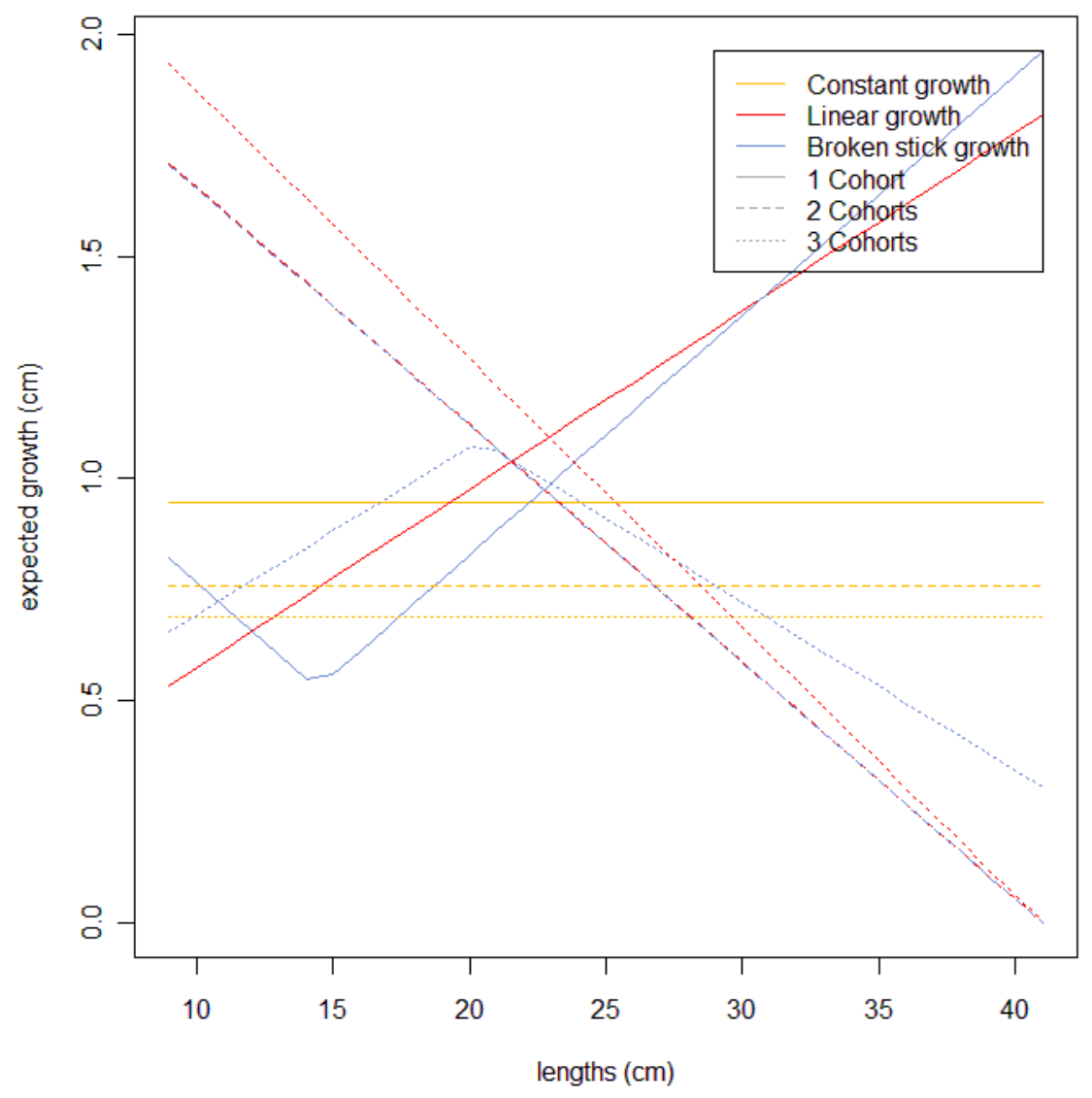

Figure 8.1.1a: Expected mean growth per week by length from models with 1, 2, and 3-cohorts and constant, linear and broken stick mean growth for Snares 2008. The colour of each line indicates the mean growth function and the type of line indicates the number of cohorts. The dotted lines are for the 3cohort models. The dashed lines are the 2-cohort models. Note that linear and broken stick 2-cohort models share the same line. The solid lines are the 1-cohort models. 

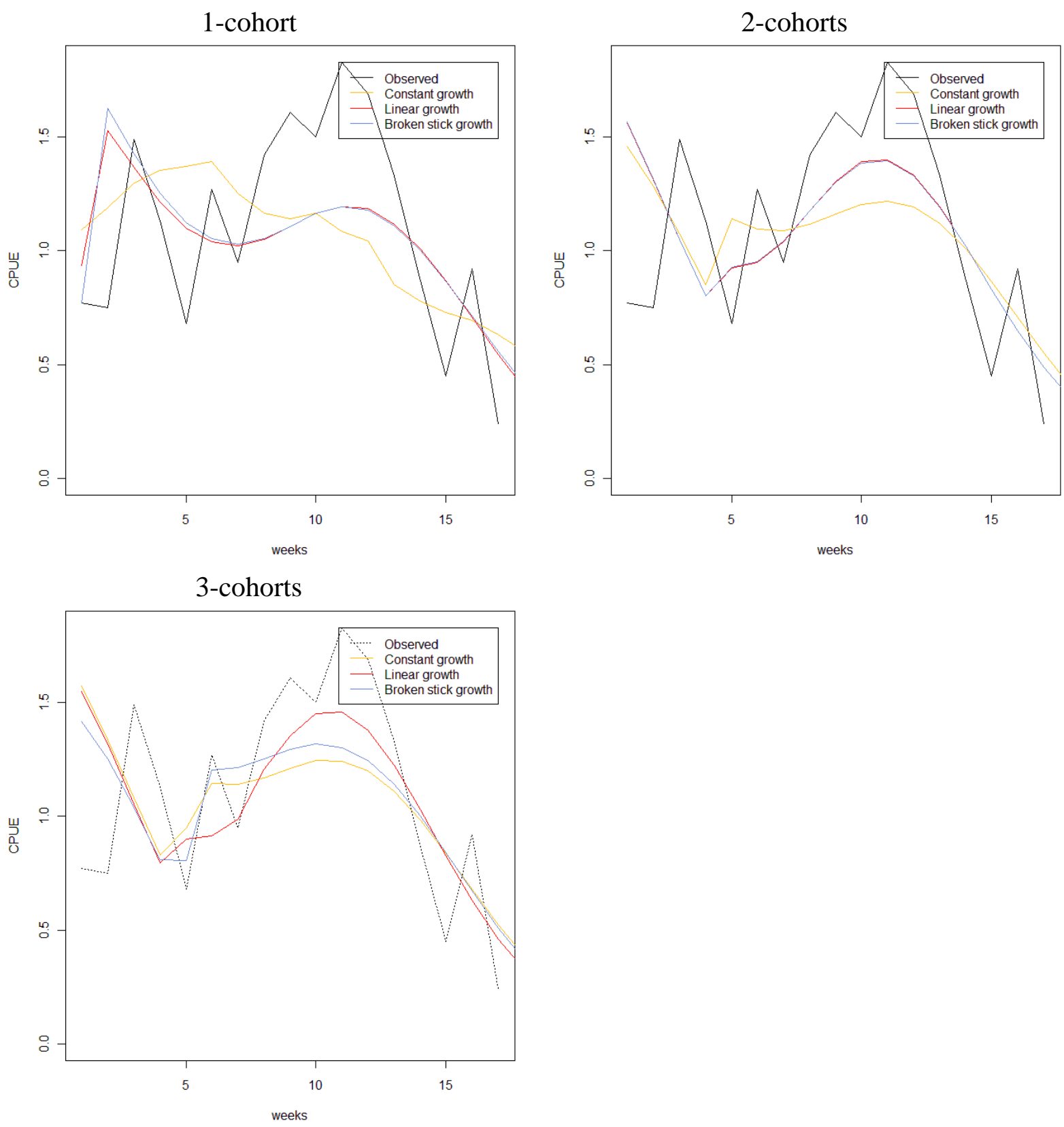

Figure 8.1.1b: Observed CPUE compared to expected CPUE for models with 1, 2, and 3-cohorts and constant, linear and broken stick mean growth for Snares 2008. 
1-cohort
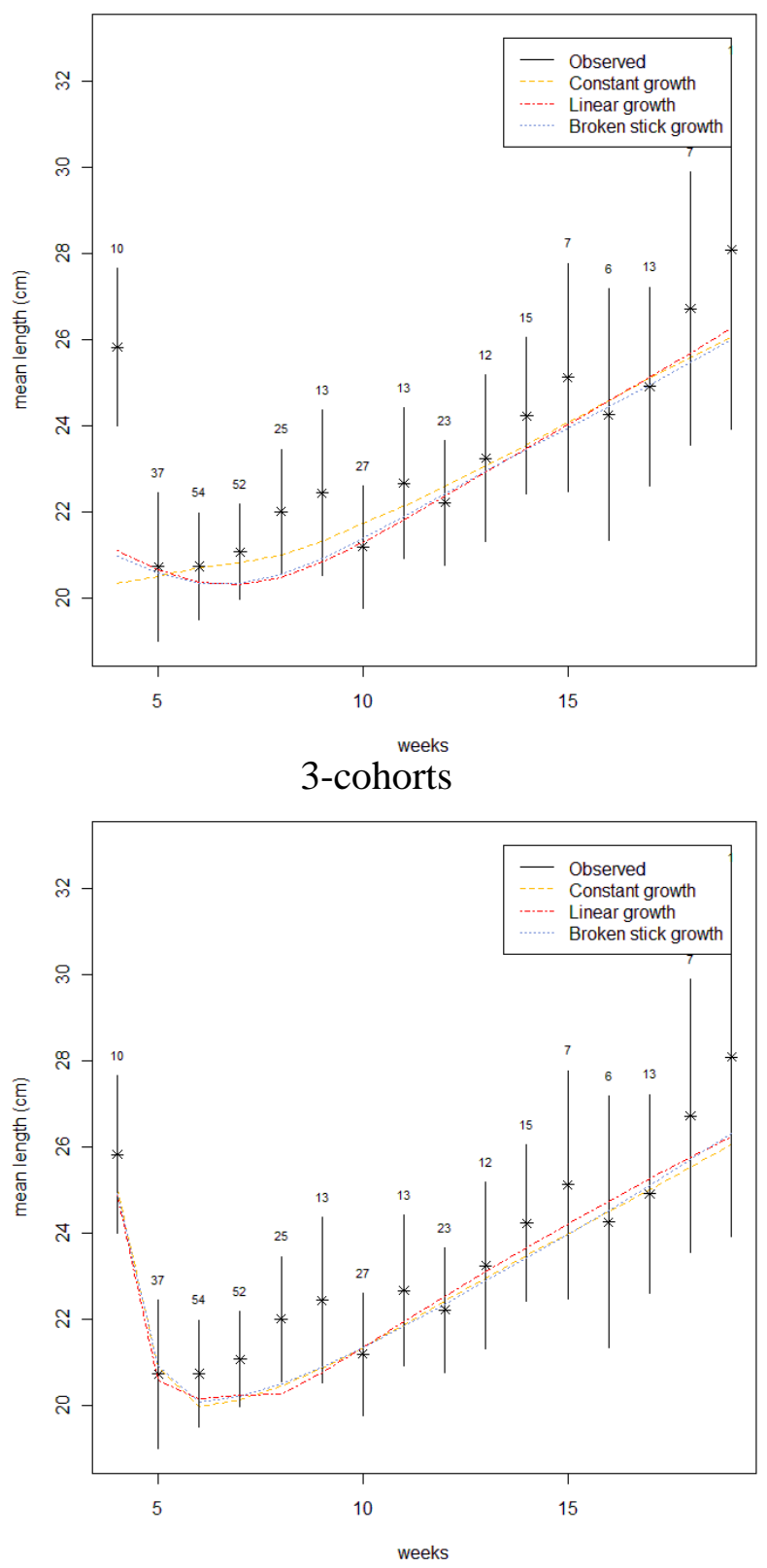

2-cohorts

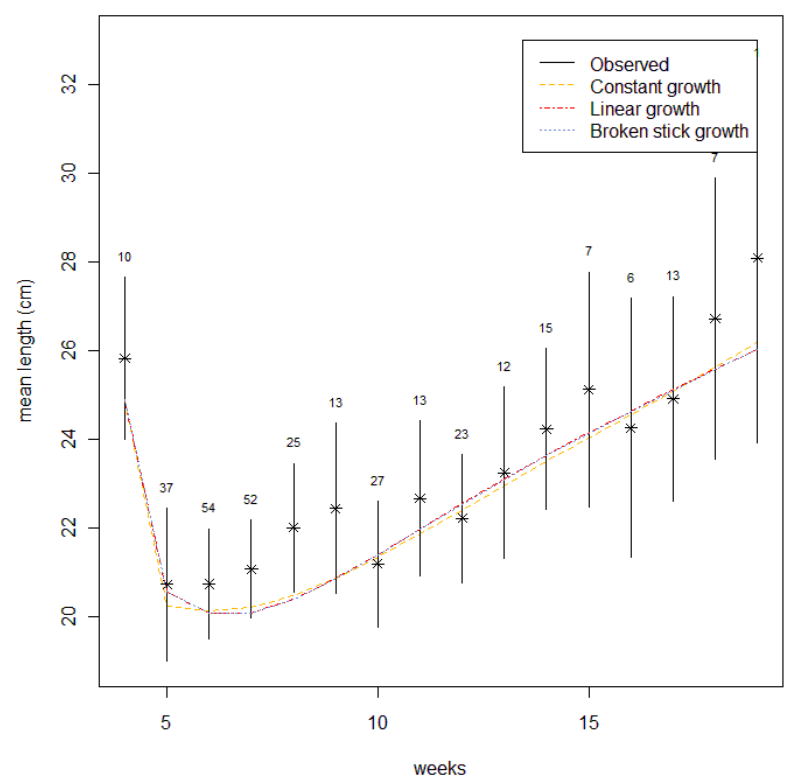

Figure 8.1.1c: Observed mean lengths with $95 \%$ confidence intervals, compared to expected mean lengths for models with 1, 2, and 3-cohorts and constant, linear and broken stick mean growth for Snares 2008. The vertical black lines show the $95 \%$ confidence intervals for the observations, calculated using the number of tows (the numbers above the black lines) as the actual sample sizes. The asterisks are the mean lengths from the data. 
1-cohort
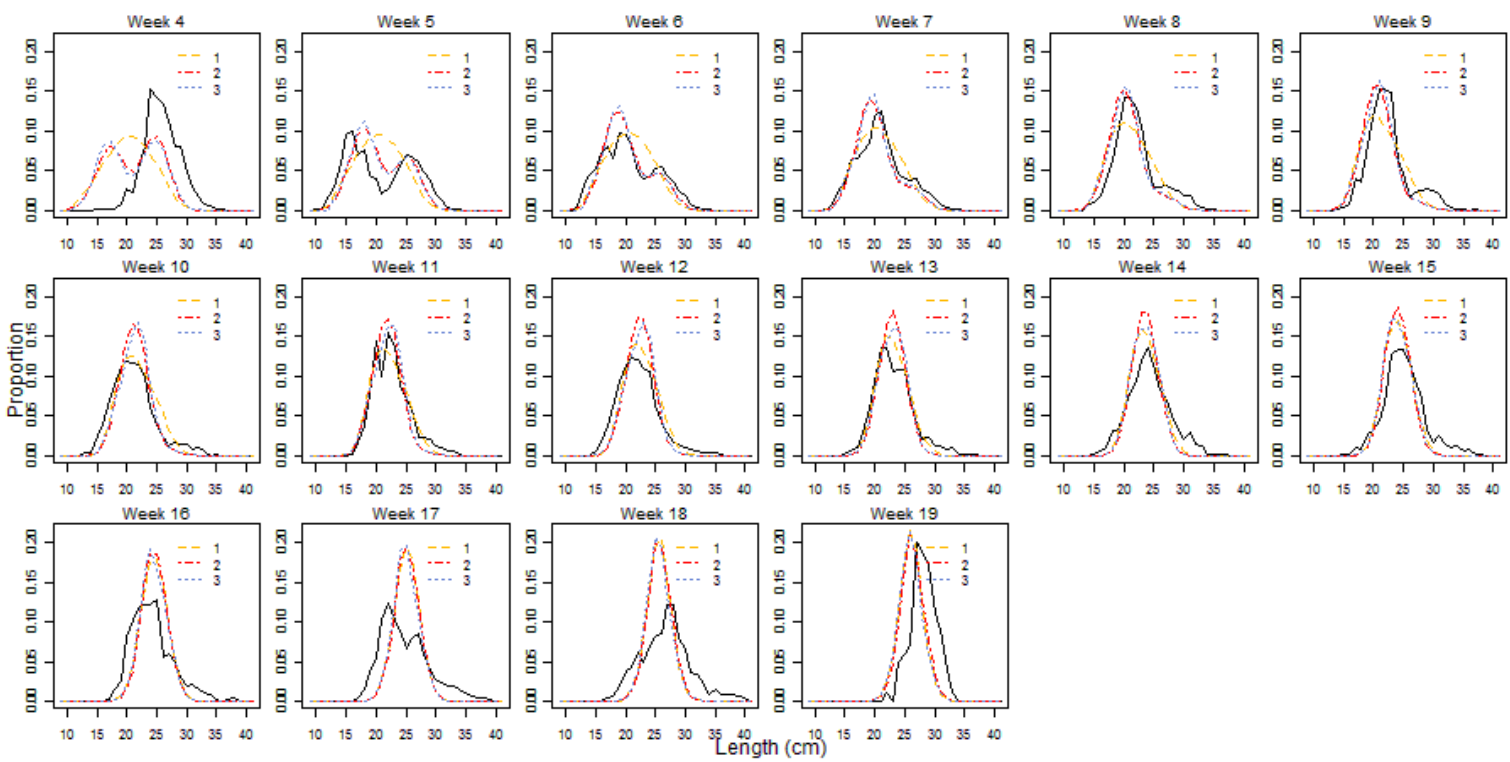

2-cohorts
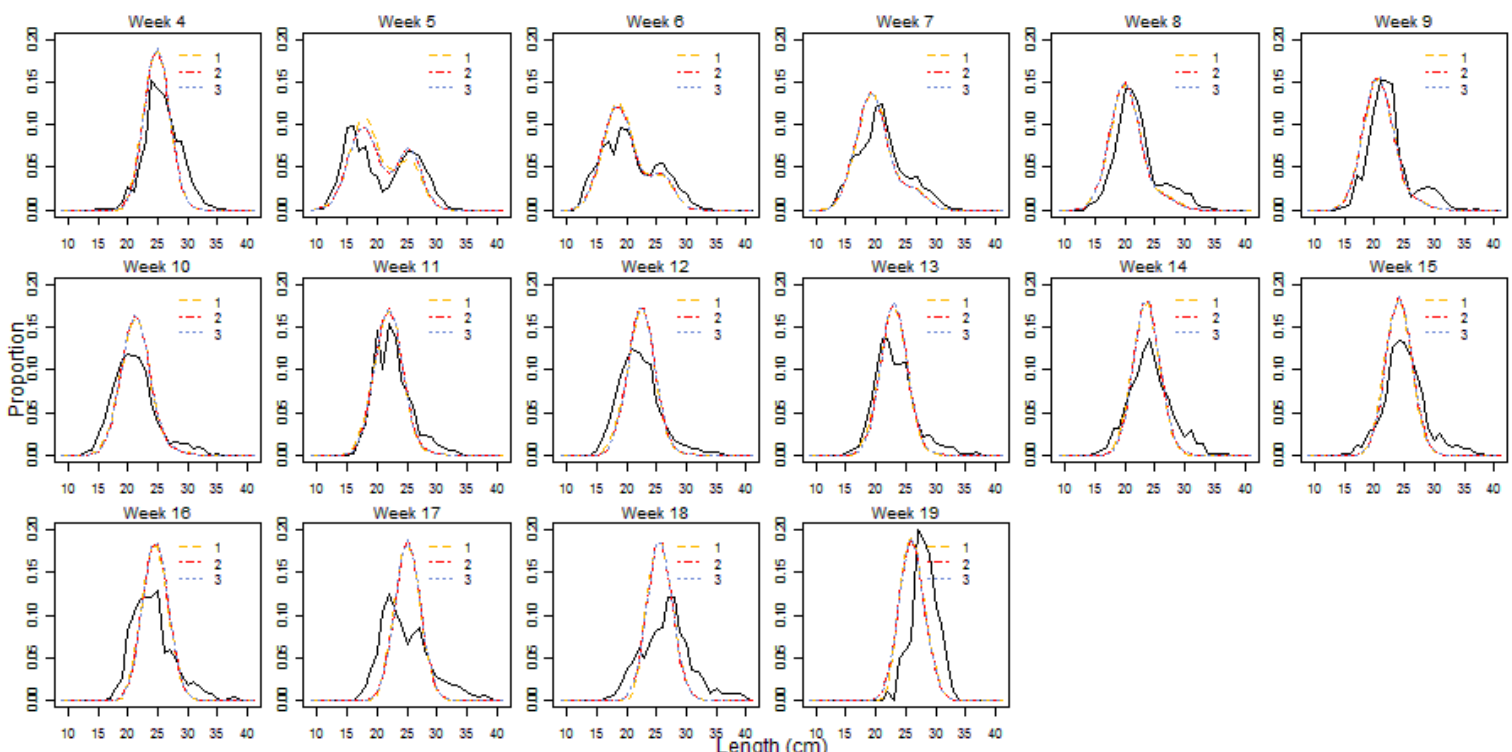

3-cohorts
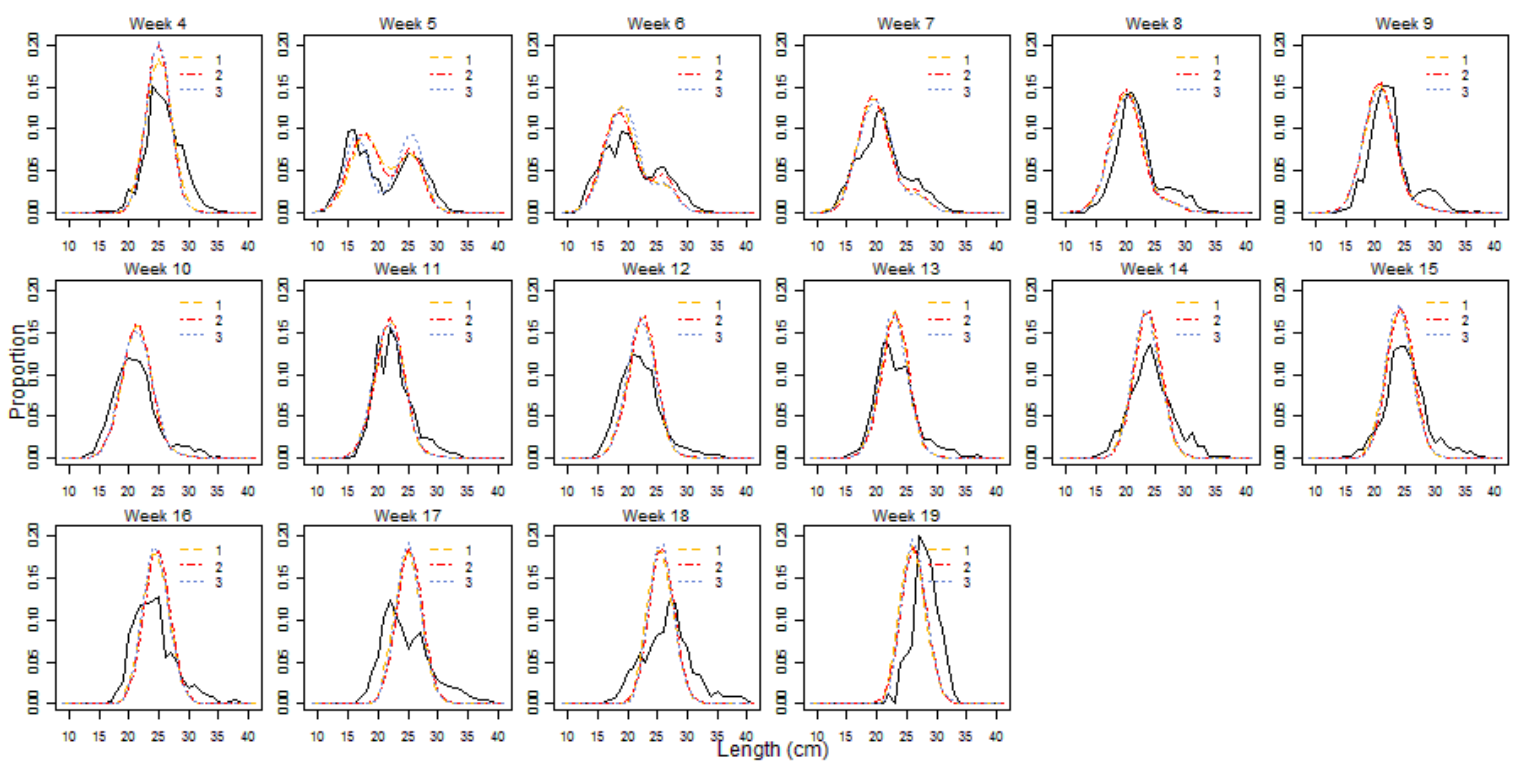

Figure 8.1.1d: Observed length frequencies compared to expected length frequencies for models with 1, 2, and 3-cohorts and constant (1), linear (2) and broken stick (3) mean growth for Snares 2008. 
A model using combined data from the Snares and Auckland Islands for 2008 was run to determine whether it would provide any evidence to support the idea that the two areas consist of the same stock. The CPUE indices were merged using a weighting system based on the catch weight in each area for each week such that for each week

$$
C P U E=C P U E_{s}\left(\frac{\text { catch }_{s}}{\text { catch }_{t}}\right)+C P U E_{a}\left(\frac{\text { catch }_{a}}{\text { catch }_{t}}\right)
$$

where CPUE $E_{S}$ and catch $_{s}$ are the CPUE and total catch weight (respectively)for the Snares

CPUE $E_{a}$ and catch $_{a}$ are the CPUE and total catch weight (respectively)for the Auckland Islands

catch $_{t}$ is the total catch weight of the Snares and Auckland Islands

Length frequencies were merged in the same way. Catch weights were added together.

All models fitted to this dataset are somewhat questionable as there are quite a few peaks in the length frequency plots (Appendix B, Figure 8.1.2b) that the models do not fit. As seen in the mean length plot (Figure 8.1.2a), there is little information (which we would expect to see as some sort of trend in the mean lengths) about the mean growth. The result of this is a very messy plot of expected mean growth curves (Figure 8.1.2c), where the model fits to the data have a wide range of mean growth.

In selecting the best of these models, there is evidence suggesting a second cohort is required. The drop in the mean length plot (Figure 8.1.2a) from week 4 to week 5 requires a new cohort.

There is no significant improvement from adding a third cohort or from increasing the complexity of mean growth from constant growth.

Ideal number of cohorts: 2

Ideal mean growth function: Constant (1 parameter) 


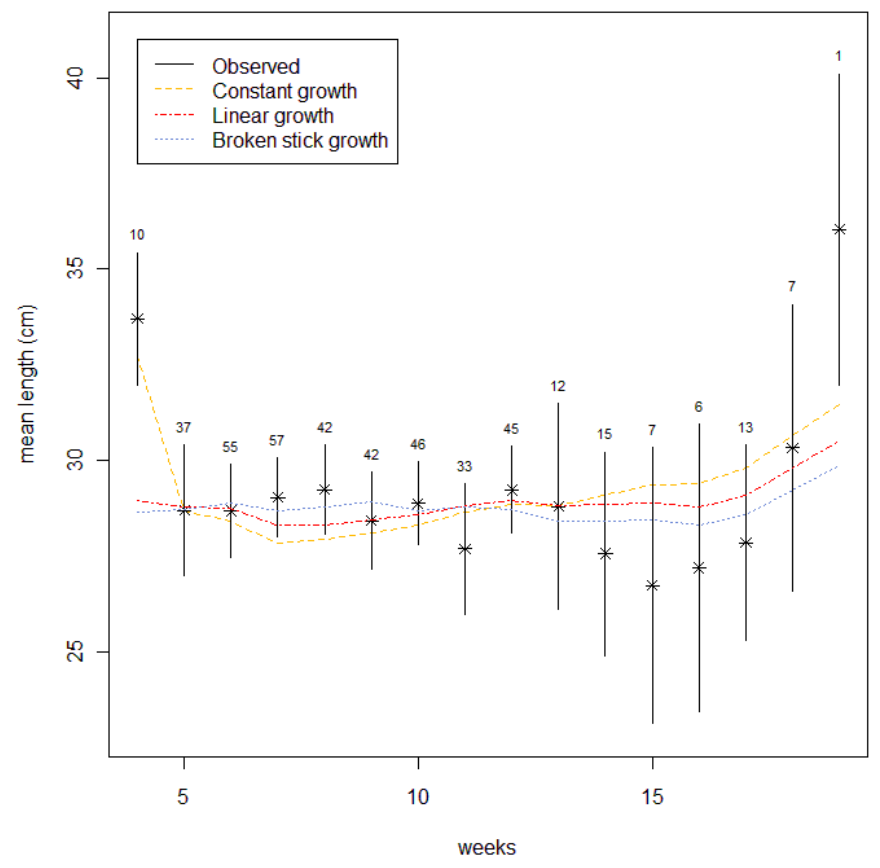

Figure 8.1.2a: Observed mean lengths with $95 \%$ confidence intervals, compared to expected mean lengths for Snares and Auckland Islands 2008, for 1, 2, and 3 mean growth parameters and 2-cohorts. The vertical black lines show the $95 \%$ confidence intervals for the observations, calculated using the number of tows (the numbers above the black lines) as the actual sample sizes.
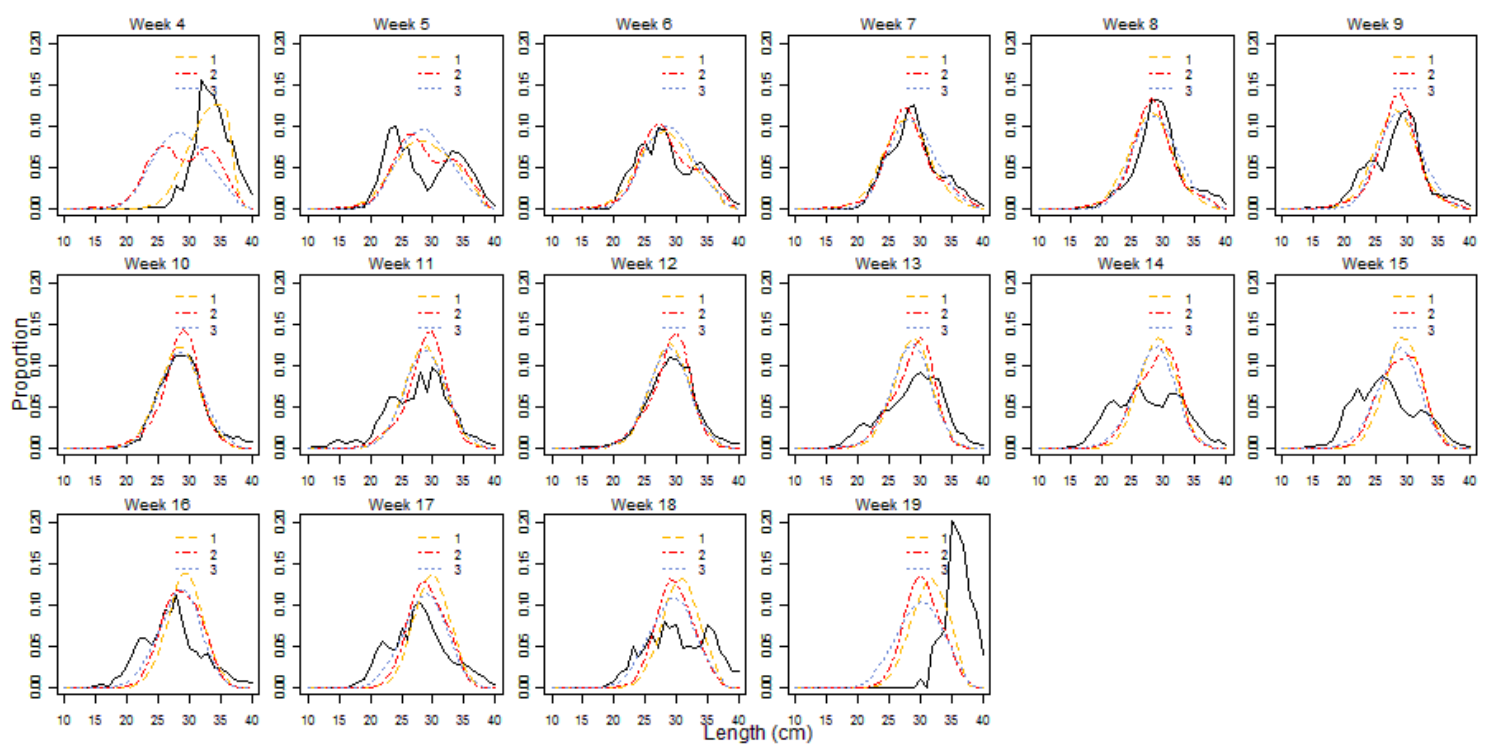

Figure 8.1.2b: Observed length frequencies against expected length frequencies for Snares and Auckland Islands 2008, for 1, 2, and 3 mean growth parameters and 2-cohorts. 


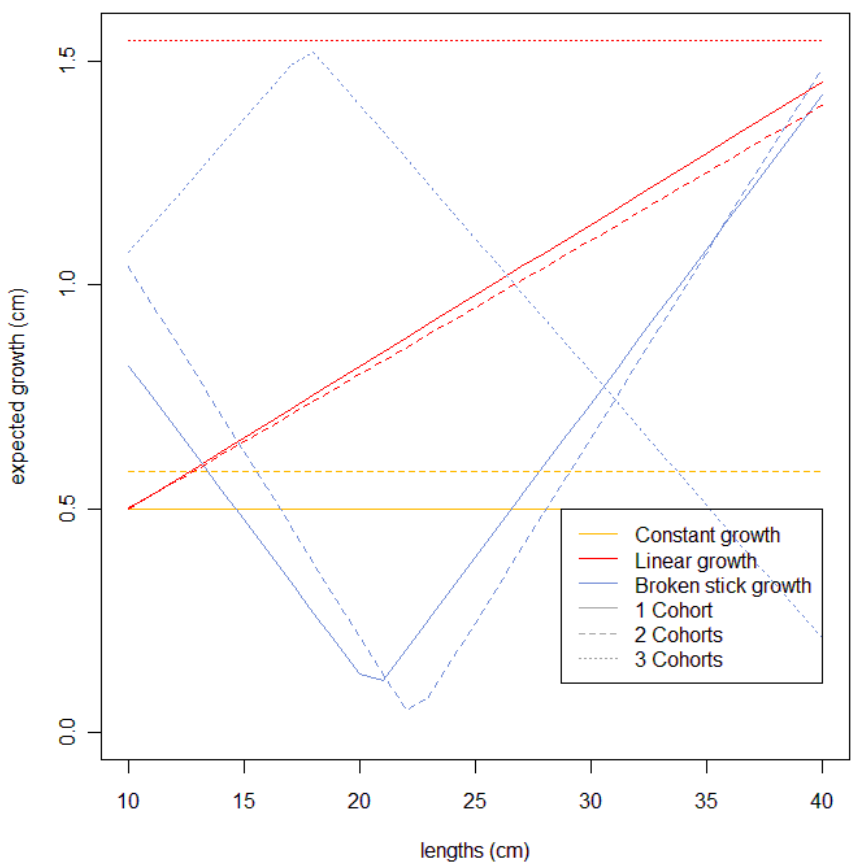

Figure 8.1.2c: Expected mean growth by length for Snares and Auckland Islands 2008 from models with 1,2 , and 3 mean growth parameters and 2-cohorts. Mean growth is the mean growth for 1 week.

\section{Snares 1991}

The model required 3-cohorts. There are two decreases in the mean length plot (Figure 8.1.3a) which only the 3-cohort models could fit to. The first is the drop in mean length from week 4 to week 5 . The second is a drop from week 12 to week 15 .

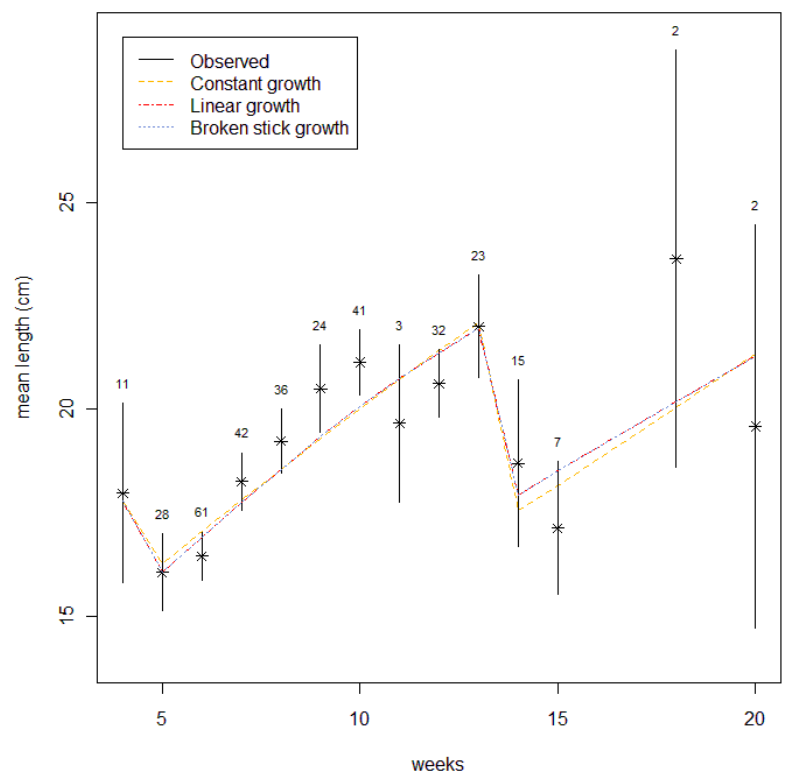

Figure 8.1.3a: Observed mean lengths with $95 \%$ confidence intervals, compared to expected mean lengths for Snares 1991, for constant, linear, and broken stick mean growth and 3-cohorts. The vertical black lines show the $95 \%$ confidence intervals for the observations, calculated using the number of tows (the numbers above the black lines) as the actual sample sizes.

There is no support for increasing the complexity of the mean growth function. The fit to the mean lengths (Figure 8.1.3a), length frequencies (Figure 8.1.3b) and CPUE (Figure 8.1.3d) all show very little improvement. The model fits very poorly to the CPUE in all cases. In fact, the best fits to CPUE come from the 1-cohort models (Figure 8.1.3c). This suggests some conflict between the CPUE data and length frequency data. 
Ideal number of cohorts: 3

Ideal mean growth function: Constant (1 parameter)

(a)

(b)
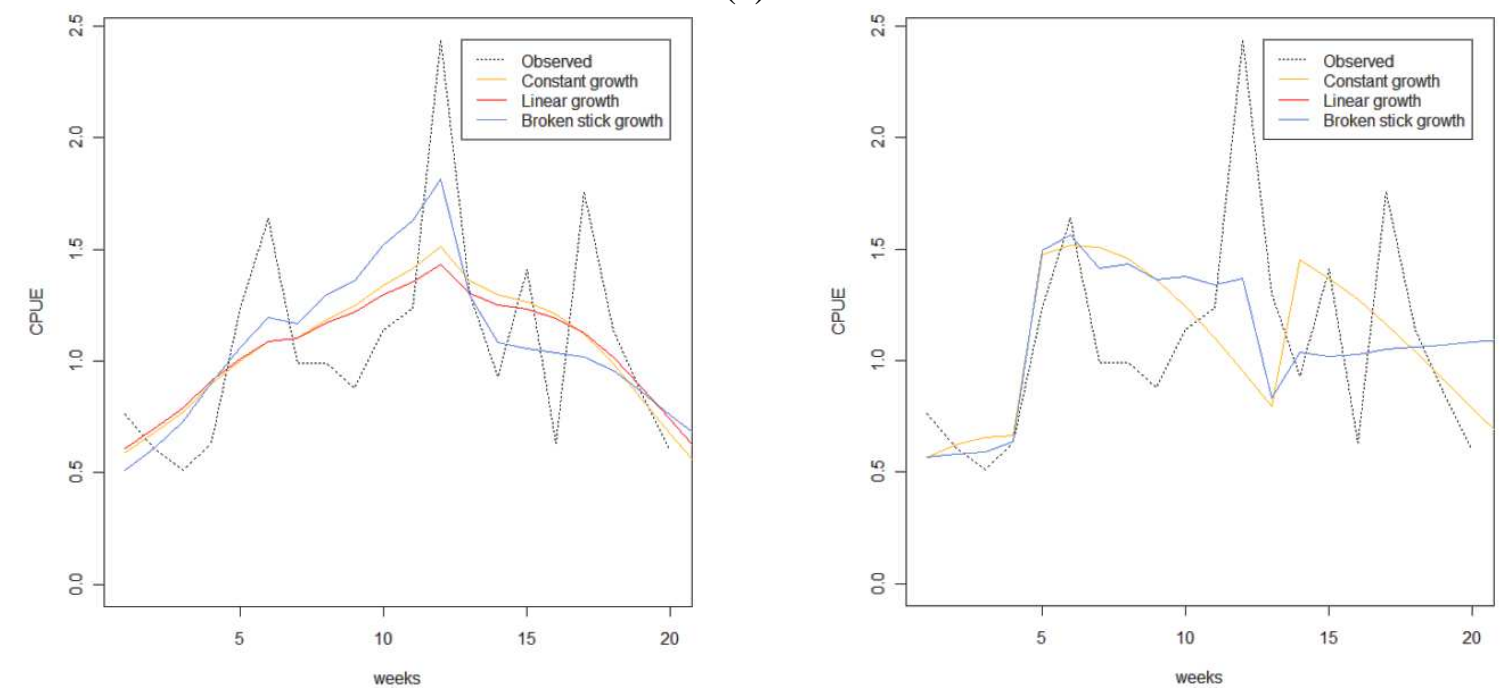

Figure 8.1.3: Observed CPUE compared to expected q $\times$ exploitable biomass for Snares 1991, for 1, 2, and 3 mean growth parameters and (a) 1-cohort (b) 3-cohorts. Note, that the linear growth and broken stick growth models share the same line, hence only the broken stick line is visible.

\section{Snares 1990}

A second cohort was required to fit the model. The mean length plot (Figure 8.1.4a) shows a decrease in mean length somewhere after week 5 and before week 11 which requires a new cohort. There are no length frequency data for the weeks in between 5 and 11 . There is no significant improvement from adding a third cohort, or from increasing the complexity of the mean growth function beyond constant. The model fits the CPUE well (Figure 8.1.4b). The number of tows sampled is quite low for each week (Table 5.1), so the length frequency data are given less weighting, which gives the CPUE more effect.

\section{Ideal number of cohorts: 2}

Ideal mean growth function: Constant (1 parameter) 


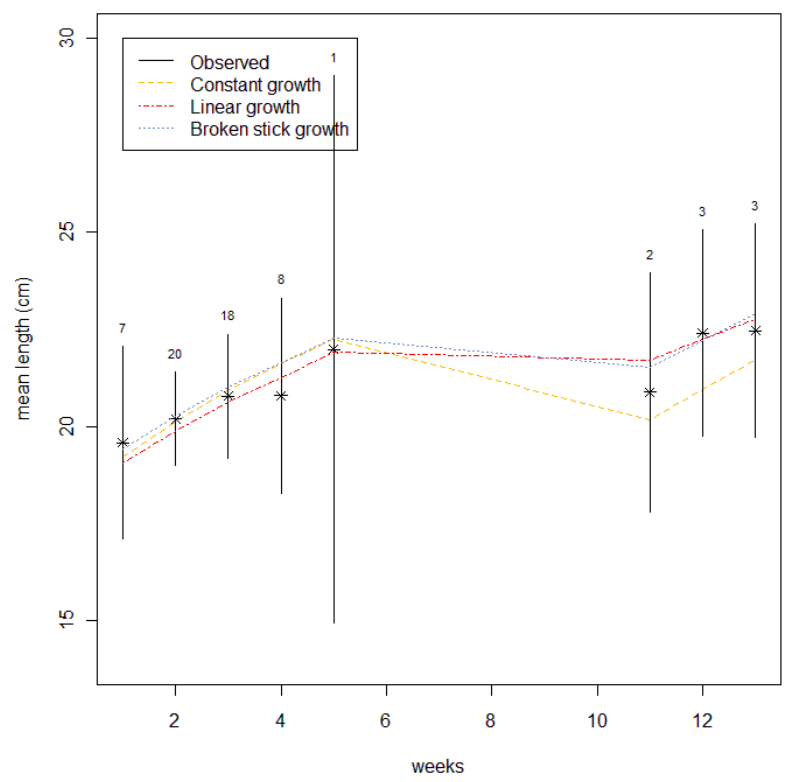

Figure 8.1.4a: Observed mean lengths with $95 \%$ confidence intervals, compared to expected mean lengths for Snares 1990, for constant, linear and broken stick mean growth and 2-cohorts. The vertical black lines show the $95 \%$ confidence intervals for the observations, calculated using the number of tows (the numbers above the black lines) as the actual sample sizes.

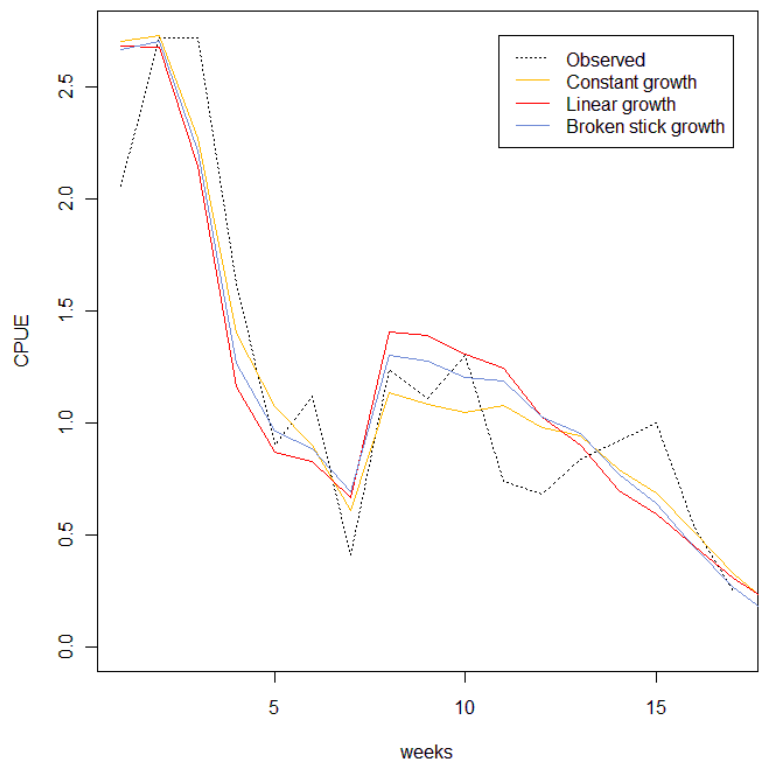

Figure 8.1.4b: Observed CPUE compared to expected q $\times$ exploitable biomass for Snares 1990, for constant, linear and broken stick mean growth and 2-cohorts. 


\section{Chapter 9: Discussion}

\section{1: Comparison of fitted models}

For each of the four area and season combinations, the best model was selected on the basis of the number of recruitment cohorts and the best function for the mean growth per week (Chapter 8.1). This section compares the parameter values for these best models. Where a parameter takes similar values across all datasets, it may suggest this parameter could be held fixed in future modelling of this fishery. Where a key parameter varies significantly, it may indicate possible inconsistencies between the models, raising questions as to the reliability of the results from the models.

The mean growth function selected was constant growth in all cases. This does not suggest the squid in the fishery for the given seasons grew at a constant rate. It simply means that there was insufficient data supporting or requiring a more complex mean growth function. For future models of this fishery I recommend offering more complex functions for mean growth.

The values of mean growth for all years in the Snares were similar, ranging from $0.78 \mathrm{~cm}$ to $1.10 \mathrm{~cm}$ per week (Table 9.1a). When the Auckland Islands and Snares data for 2008 were combined, the mean growth was much lower at $0.58 \mathrm{~cm}$ per week. This is to be expected, as including the Auckland Islands data meant more fish in the later weeks, requiring the model to retain more of the population at these lengths.

Table 9.1a: Expected parameter values from 'best' models as selected in section 8.1 for the four area/year combinations; Snares 2008, 1991, 1990 and Snares and Auckland Islands combined 2008.

\begin{tabular}{|c|c|c|c|c|c|c|c|c|c|}
\hline $\begin{array}{c}\text { Area and } \\
\text { year }\end{array}$ & $\begin{array}{c}\text { Number } \\
\text { of } \\
\text { cohorts }\end{array}$ & $\begin{array}{l}\text { Mean } \\
\text { growth } \\
\text { function }\end{array}$ & $\begin{array}{l}\text { Mean } \\
\text { growth } \\
(\mathrm{cm})^{-w}\end{array}$ & $\begin{array}{l}\mu_{\text {sel }} \\
(\mathrm{cm})\end{array}$ & $\sigma_{\text {selL }}$ & $\sigma_{\text {SelR }}$ & $\begin{array}{l}\mu_{\text {recruit }} \\
\text { (cm) }\end{array}$ & $\sigma_{\text {recruit }}$ & $\begin{array}{c}\text { Escapem } \\
\text { ent }\end{array}$ \\
\hline Snares 2008 & 2 & Constant & 0.76 & 22.00 & 5.61 & 3.60 & 15.82 & 2.96 & 0.89 \\
\hline $\begin{array}{r}\text { Snares and } \\
\text { Auckland } \\
\text { Islands } 2008\end{array}$ & 2 & Constant & 0.58 & 35.84 & 6.56 & 1.23 & 17.99 & 4.92 & 0.63 \\
\hline Snares 1991 & 3 & Constant & 0.92 & 14.25 & 5.78 & 6.20 & 15.28 & 1.18 & 0.95 \\
\hline Snares 1990 & 2 & Constant & 1.10 & 24.06 & 12.14 & 0.80 & 16.04 & 2.09 & 0.83 \\
\hline $\begin{array}{r}\text { Mean or } \\
\text { mode }\end{array}$ & 2 & Constant & 0.84 & 24.04 & 7.52 & 2.96 & 16.28 & 2.79 & 0.83 \\
\hline $\begin{array}{l}\text { Standard } \\
\text { deviation }\end{array}$ & & & 0.22 & 8.93 & 3.11 & 2.49 & 1.18 & 1.60 & 0.14 \\
\hline
\end{tabular}

The mean length at selectivity ranged from $14.25 \mathrm{~cm}$ to just over $24 \mathrm{~cm}$ for the Snares and increased to $35.84 \mathrm{~cm}$ for the Snares and Auckland Islands 2008 combined. This range is large and does not suggest a preferred value that could be used for future models. Further, a significantly different mean length for the selectivity alters the whole parameter space of the model and so it becomes difficult to draw conclusions that can be applied to future models. For example, compare the timing of the second cohort to enter the population and the mean of its length distribution. A season modelled with a second cohort coming in at about week 4 with mean length of approximately $14 \mathrm{~cm}$ and mean length at selectivity of approximately 14 
$\mathrm{cm}$ is going to be very different to one with the same second cohort timing and mean recruitment length, but a mean length at selectivity of $24 \mathrm{~cm}$. The new cohort will have much less effect in this second model but will be significant in the first. The escapement should be relatively insensitive to varying mean lengths at selectivity. Once recruitment comes into the population, most of it remains there until it is selected, after which a proportion is caught.

The mean length at recruitment was consistent in the Snares models and somewhat higher for the Snares and Auckland Islands 2008 combined. However, the left and right standard deviations vary considerably.

Escapement, which is the key variable output from these models, is relatively stable. For the Snares, it ranges from 0.83 to 0.95 (Table 9.1a) and is considerably lower at 0.63 for the Snares and Auckland Islands 2008 combined.

\section{2: The modelling approach used and future recommendations}

The post-season model for Arrow squid in the Snares and Auckland Islands investigated here is an integrated model that fits to the CPUE indices, length frequency data and catch weights. It treats the observations statistically, allowing for outliers. The key output is the calculated escapement rate. Also note-worthy are the fit to the CPUE, the estimated growth rates and the estimated number of cohorts.

The escapement rates across the 'best' models compared in Chapter 9.1 seem relatively stable with a range of 0.83-0.95. This is promising for using this modelling approach for postseason modelling of the squid fishery. Compared with the common management target of $40 \%$ escapement (Beddington et al., 1990), the 83-95\% calculated here could, with further testing on more seasons, suggest the fishery is currently underexploited.

The CPUE was not convincingly fitted by the models in most cases. It is possible the CPUE is not proportional to biomass, which is an assumption made in the model. It is also possible the length frequency data are not representative of the catches, forcing the model to underfit the CPUE in order to fit the length frequencies. In a preliminary analysis, Hurst et al. (2012) found evidence suggesting the CPUE could predict years of high catches by week 8 . Given the poor fits to the CPUE when modelled together with the length frequency data, the CPUE alone may not be such a reliable indicator.

The growth for all 'best' models was found to be constant with means ranging from $0.76 \mathrm{~cm}$ per week to $1.10 \mathrm{~cm}$ per week. The growth rates determined by Uozumi (1998) were not constant, but are in a similar range to this. Hurst et al. (2012) estimated growth for Snares 2008 at $0.75-1.25 \mathrm{~cm}$ per week which is also similar. Future models could assume constant growth in this range, although I suspect length frequency data sampled from a greater number of tows each week may give more information on the growth and fit better to linear decreasing growth.

Due to the similarity between fish lengths from the same catch (Francis, 2011), it would be better if observations for the length frequency data focussed on sampling a large number of tows (and hence catches) rather than a large number of squid, potentially from only a few catches. 
The model could be improved if it incorporated more sources of variation, allowing for a range of feasible values for the length to weight conversion. This would create more variation in the results. Simulations could be run to decide the importance of this variation, whether it makes it harder to detect cohorts and what effect it has on calculating the escapement.

Uozumi (1998) found evidence that the squid grow at different rates based on when they spawn. This model may benefit from keeping the cohorts separate throughout the time steps so they can grow at different rates. It is unlikely the growth information in the data analysed here is strong enough to support growth that varies by cohort, given it didn't require it to vary by length (constant growth was selected in all cases). However, it may be beneficial if this modelling approach is to be used for post-season modelling of future seasons which may use more suitable sampling of the catches for the length frequency data. 


\section{References}

Augustyn, C. J., \& Roel, B. A. (1998). Fisheries biology, stock assessment, and management of the chokka squid [Loligo vulgaris reynaudii] in South African waters: An overview. California Cooperative Oceanic Fisheries Investigations Reports, 39, 71-80.

Beddington, J. R., Rosenberg, A. A., Crombie, J. A., \& Kirkwood, G. P. (1990). Stock Assessment and the Provision of Management Advice for the Short Fin Squid Fishery in Falkland Islands Waters. Fish. Res., 8, 351-365.

Bull, B., Francis, R. I. C. C., Dunn, A., McKenzie, A., Gilbert, D. J., Smith, M. H., \& Brian, R. (2008) CASAL ( +++ elgorithmis stock assessment laboratory): CASAL User Manual v2.20-2008/02/14. NIWA Technical Report $130275 p$.

Caddy, J. F. (1996). Modelling natural mortality with age in short-lived invertebrate populations: definition of a strategy of gnomonic time devision. Aquat. Living Resour., 9, 197-207.

Campbell, R. A. (2004). CPUE standardisation and the construction of indices of stock abundance in a spatially varying fishery using general linear models. Fish. Res., 70(2-3), 209-227.

Chen, X. J., Chen, Y., Tian, S. Q., Liu, B. L., \& Qian, W. G. (2008). An assessment of the west winter-spring cohort of neon flying squid (Ommastrephes bartramii) in the Northwest Pacific Ocean. Fisheries Research, 92(2-3), 221-230. doi: DOI 10.1016/j.fishres.2008.01.011

Cochran, W. G. (1977). Sampling Techniques. United States of America and simultaneously in Canada: John Wiley \& Sons, Inc.

Dunn, A. (2002). Updated catch-per-unit-effort indices for hoki (Macruronus novaezelandiae) on the west coast South Island, Cook Strait, Chatham Rise, and sub-Antarctic for the years 1990 to 2001 New Zealand Fisheries Assessment Report (Vol. 47, pp. 51).

Fournier, D. A., Sibert, J. R., Majkowski, J., \& Hampton, J. (1990). MULTIFAN a LikelihoodBased Method for Estimating Growth Parameters and Age Composition from Multiple Length Frequency Data Sets Illustrated using Data for Southern. Canadian Journal of Fisheries and Aquatic Sciences, 47(2), 301-317.

Fournier, D. A., Skaug, H. J., Ancheta, J., Ianelli, J., Magnusson, A., Maunder, M. N., ... Sibert, J. (2011). AD Model Builder: using automatic differentiation for statistical inference of highly parameterized complex nonlinear models. Optimization Methods and Software, 27, 233-249.

Francis, R. I. C. C. (2011). Data weighting in statistical fisheries stock assessment models. Can. J. Fish. Aquat. Sci., 68, 1124-1138. doi: 10.1139/F2011-025

Hewitt, D. A., \& Hoenig, J. M. (2005). Comparison of two approaches for estimating natural mortality based on longevity. Fishery Bulletin US, 103, 433-437.

Hurst, R. J., Ballara, S. L., MacGibbon, D., \& Triantafillos, L. (2012). Fishery characteristaion and standardised CPUE analyses for arrow squid (Nototodarus gouldiI and IN. sloanii), 1989-90 to 2007-08, and potential management approaches for southern fisheries: NIWA, Wellington, NZ.

Kowalski, E. A., \& A. Meyers, P. (1997). Glacial-interglacial variations in Quaternary production of marine organic matter at DSDP Site 594, Chatham Rise, southeastern New Zealand margin. Marine Geology, 140, 249-263.

Mattlin, R. H., \& Coleman, J. A. (1988). Arrow Squid. New Zealand Fisheries Assessment Research Document, 88/34, 16 p.

Mattlin, R. H., \& Scheilbling, R. E. (1985). Distribution, Abundance and Size Structure of Arrow Squid (Nototodarus sp.) off New Zealand. NAFO Sci. Coun. Studies, 9, 39-45.

Maunder, M. N., \& Punt, A. E. (2004). Standardising catch and effort data: a review of recent approaches. Fish. Res., 70(2-3), 141-159. 
McAllister, M. K., Hill, S. L., Agnew, D. J., Kirkwood, G. P., \& Beddington, J. R. (2004). A Bayesian hierarchical formulation of the De Lury stock assessment model for abundance estimation of Falkland Islands' squid (Loligo gahi). Canadian Journal of Fisheries and Aquatic Sciences, 61(6), 1048-1059.

Ministry of Fisheries. (2011). Report from the Fisheries Assessment Plenary: stock assessments and yield estimates. Ministry of Fisheries, Wellington, New Zealand (pp. 53-64).

Pierce, G. J., \& Guerra, A. (1994). Stock assessment methods used for cephalopod fisheries. Fish. Res., 21, 255-285.

Royer, J., Peries, P., \& Robin, J. P. (2002). Stock assessments of English Channel loliginid squids: updated depletion methods and new analytical methods. Ices Journal of Marine Science, 59(3), 445-457. doi: DOI 10.1006/jmsc.2002.1203

Uozumi, Y. (1998). Fishery biology of arrow squids, Nototodarus gouldi and N. sloanii, in New Zealand waters. Bulletin of the National Institute of Far Seas Fisheries, Japan.

Young, I. A. G., Pierce, G. J., Daly, H. I., Santos, M. B., Key, L. N., Bailey, N., . . Pereira, J. M. F. (2004). Application of depletion methods to estimate stock size in the squid Loligo forbesi in Scottish waters (UK). Fish. Res., 69(2), 211-227. 
Males

Females

Total

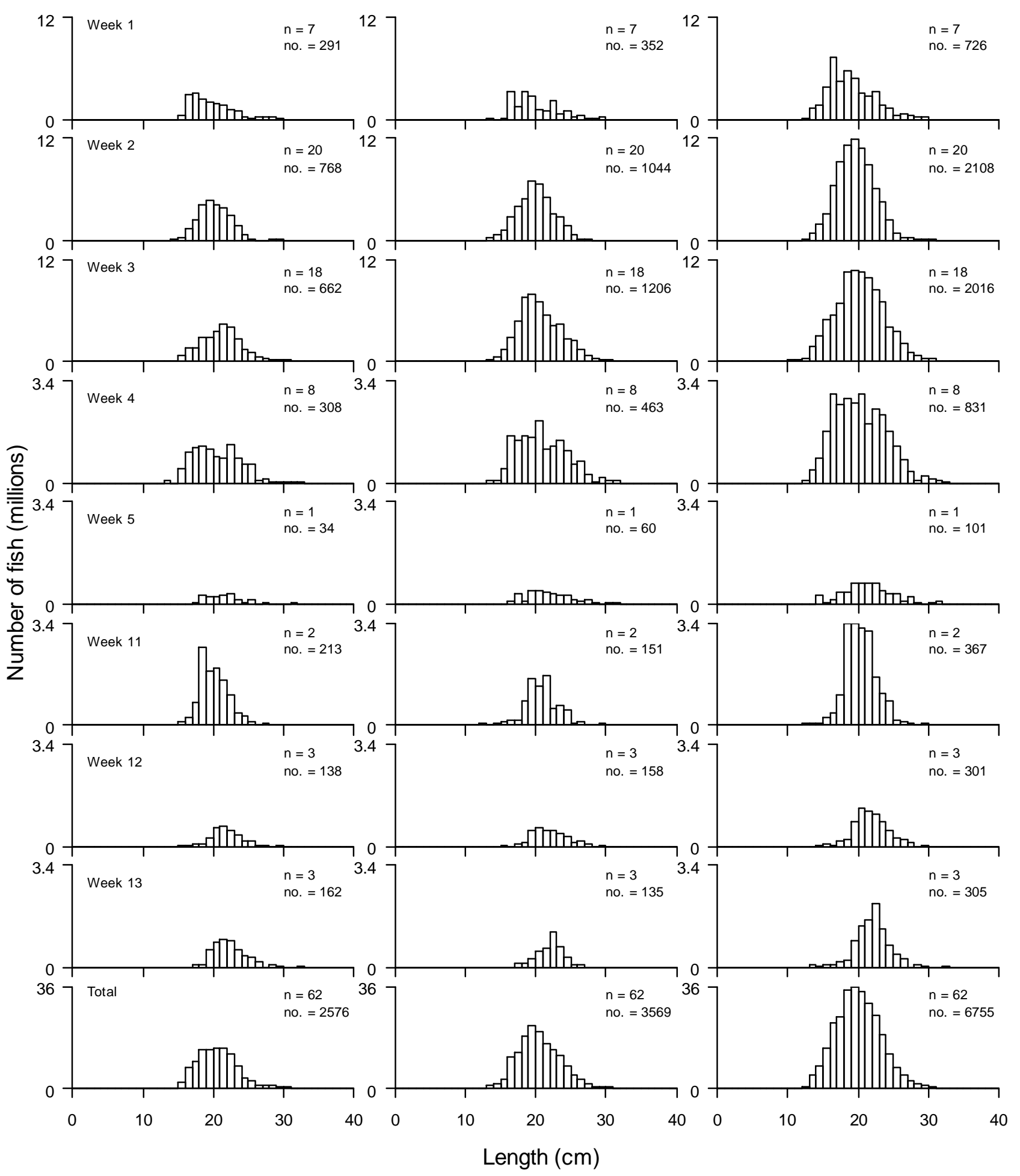

Figure A1a: Length frequency of arrow squid taken in target arrow squid commercial catches from the SNARES SHELF trawl fishery 1990 sampled by the Observer Programme. n, number of tows sampled; no., number of fish sampled (Hurst et al., 2012). 


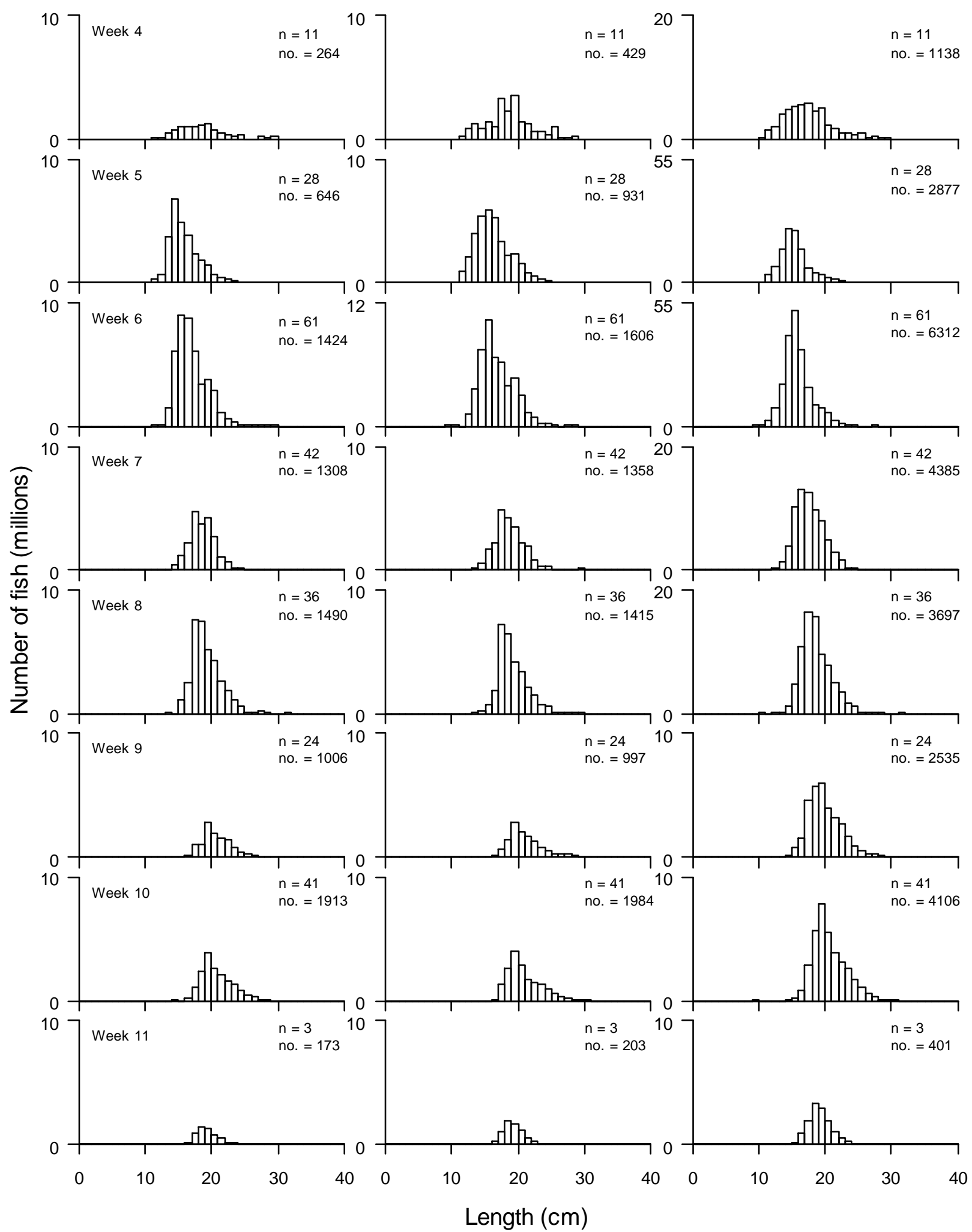

Figure A1b: Length frequency of arrow squid taken in target arrow squid commercial catches from the SNARES SHELF trawl fishery 1991 sampled by the Observer Programme. n, number of tows sampled; no., number of fish sampled (Hurst et al., 2012). 
Males

Females

Total

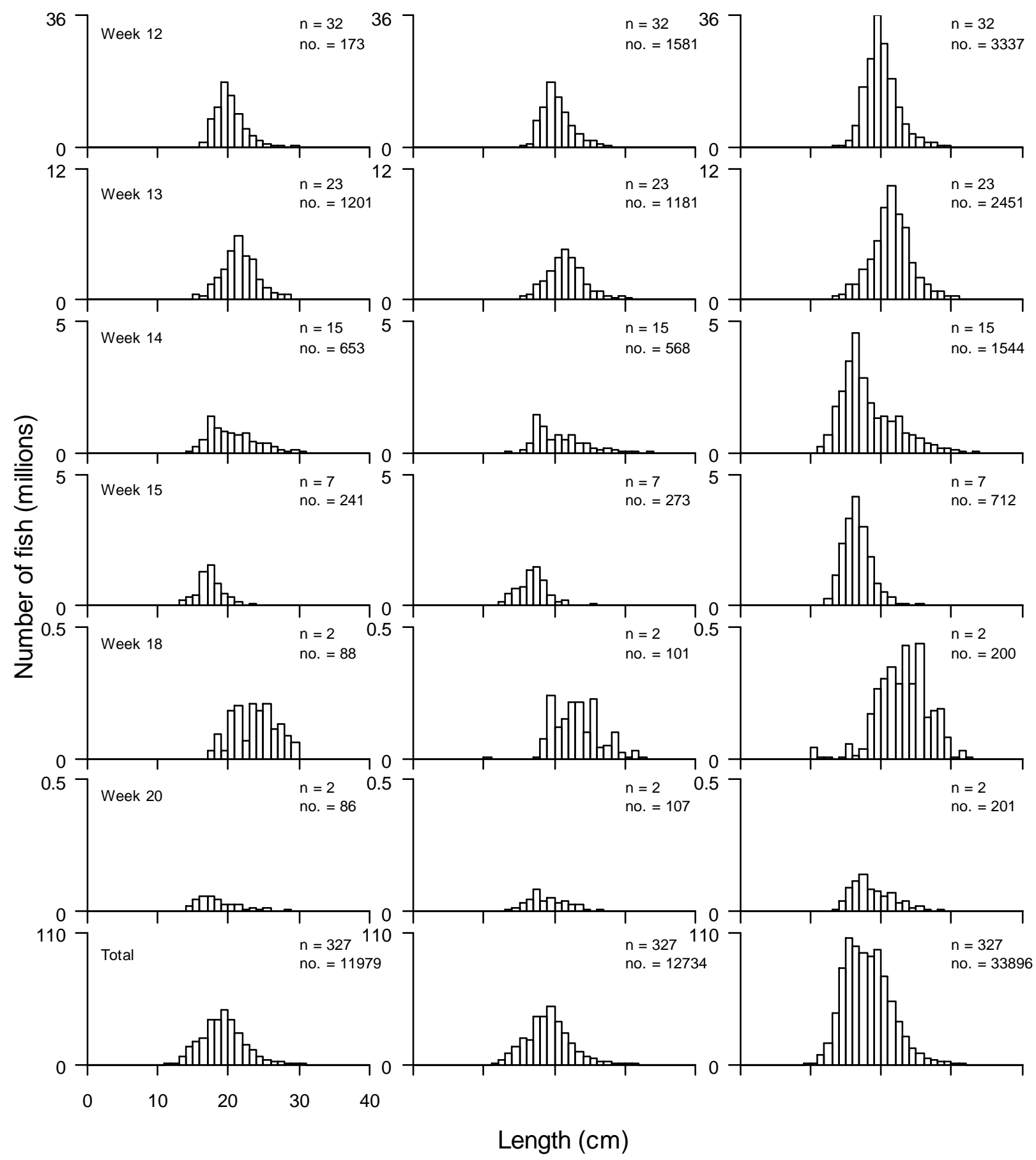

Figure A1b continued: SNARES SHELF trawl fishery 1991 length frequencies. 


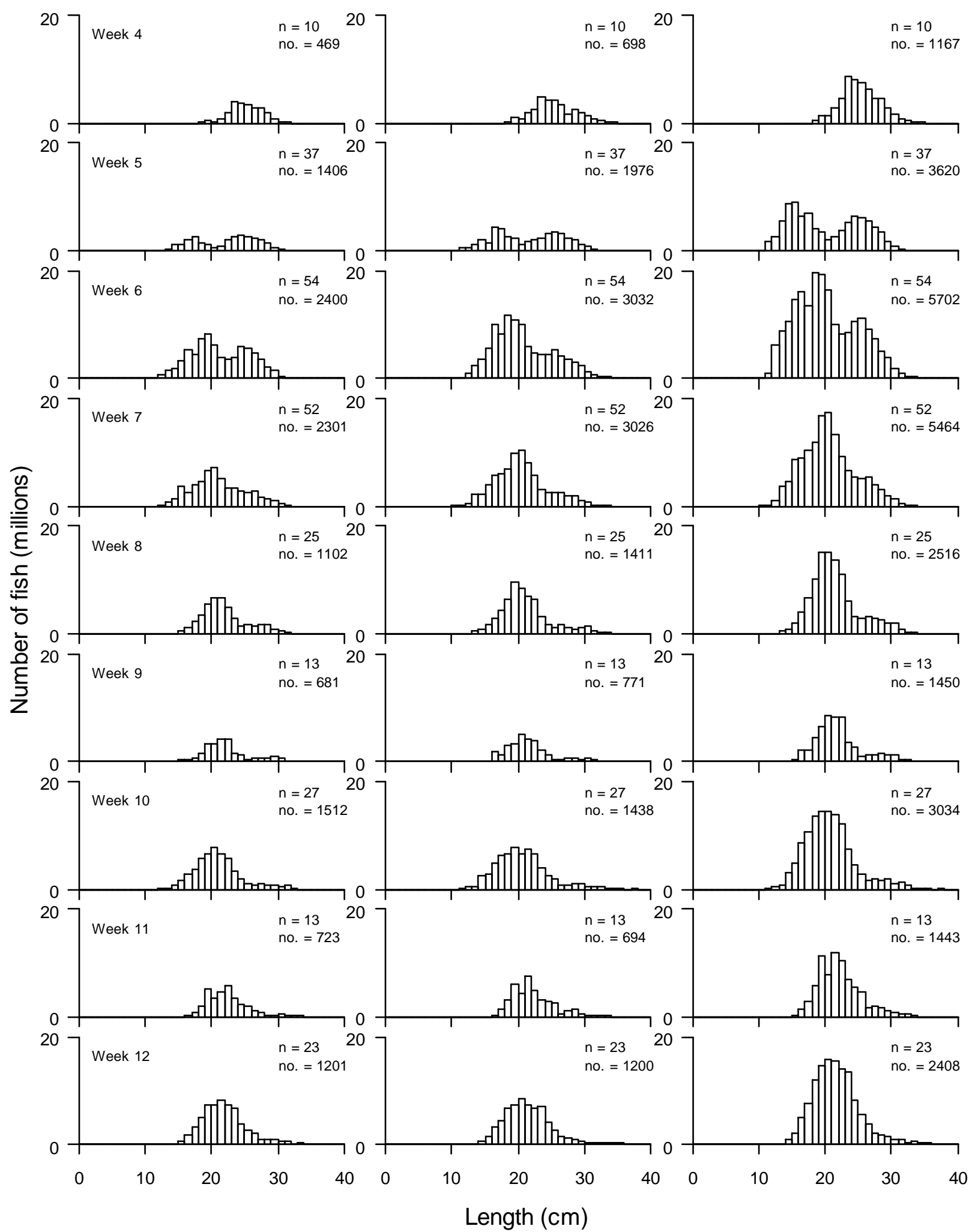

Figure A1d: Length frequency of arrow squid taken in target arrow squid commercial catches from the SNARES SHELF trawl fishery 2008 sampled by the Observer Programme. n, number of tows sampled; no., number of fish sampled (Hurst et al., 2012). 
Males

Females

Total

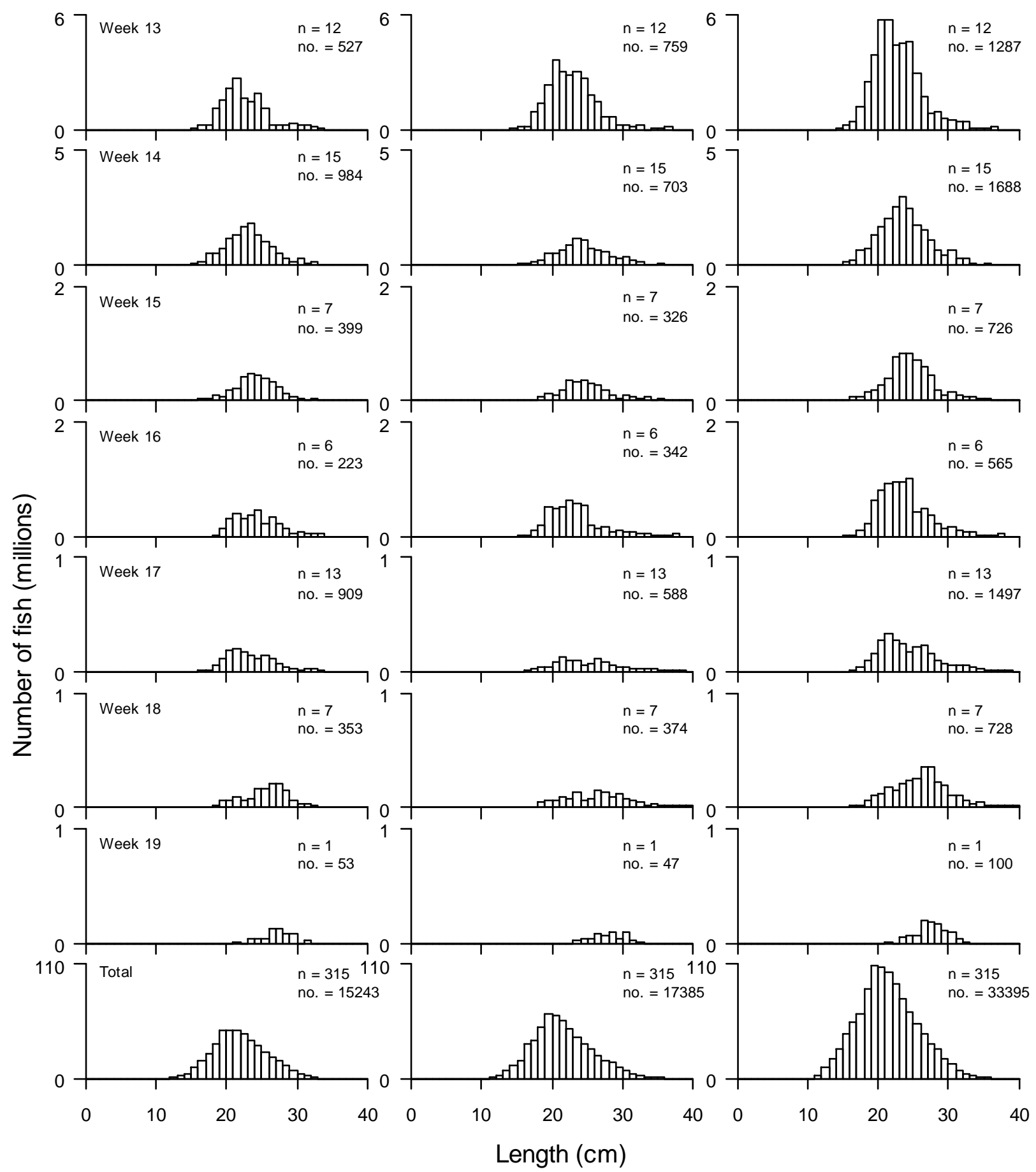

Figure A1d continued: SNARES SHELF trawl fishery 2008 length frequency. 


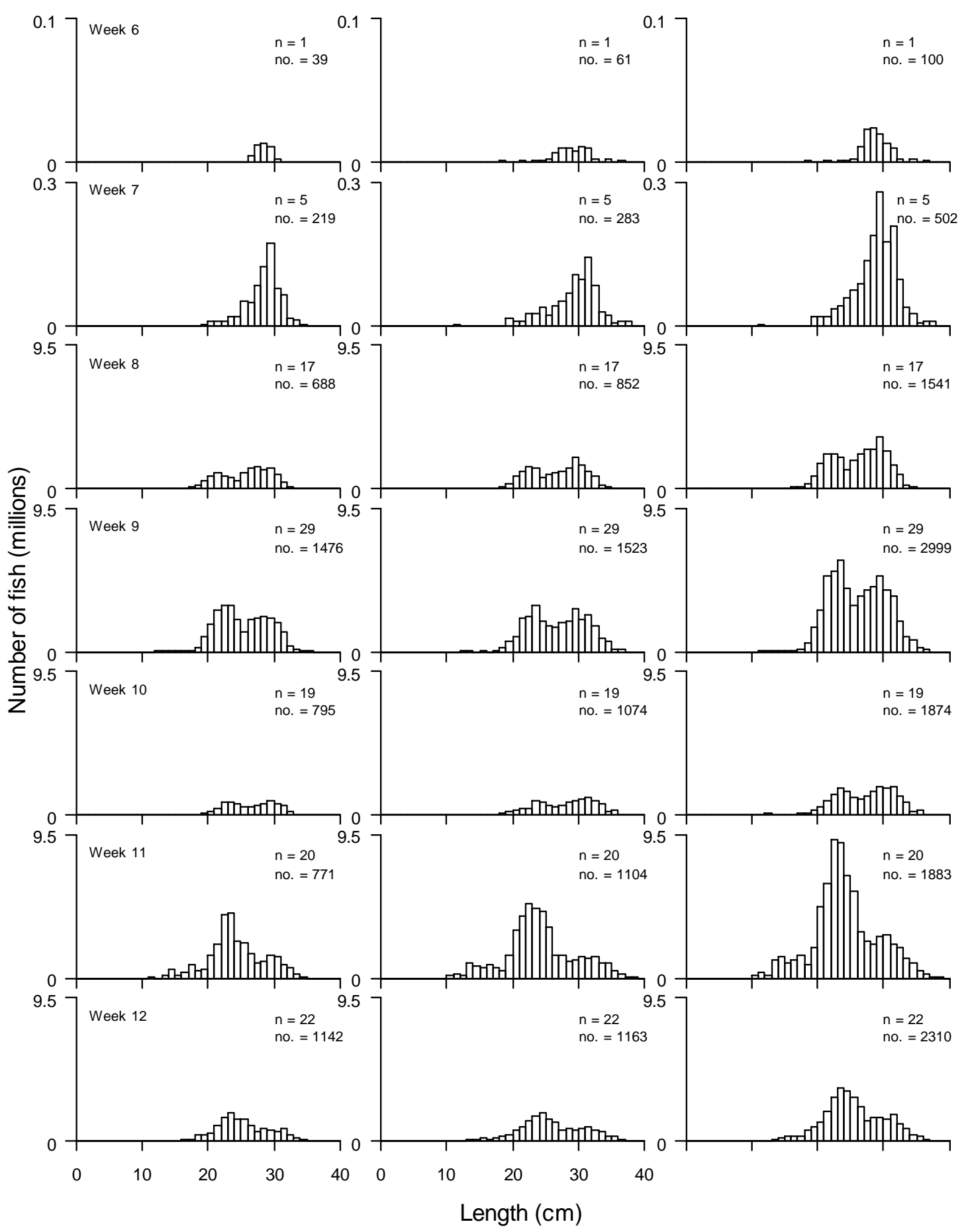

Figure A1e: Length frequency of arrow squid taken in target arrow squid commercial catches from the AUCKLAND ISLANDS trawl fishery 2008 sampled by the Observer Programme. n, number of tows sampled; no., number of fish sampled (Hurst et al., 2012). 


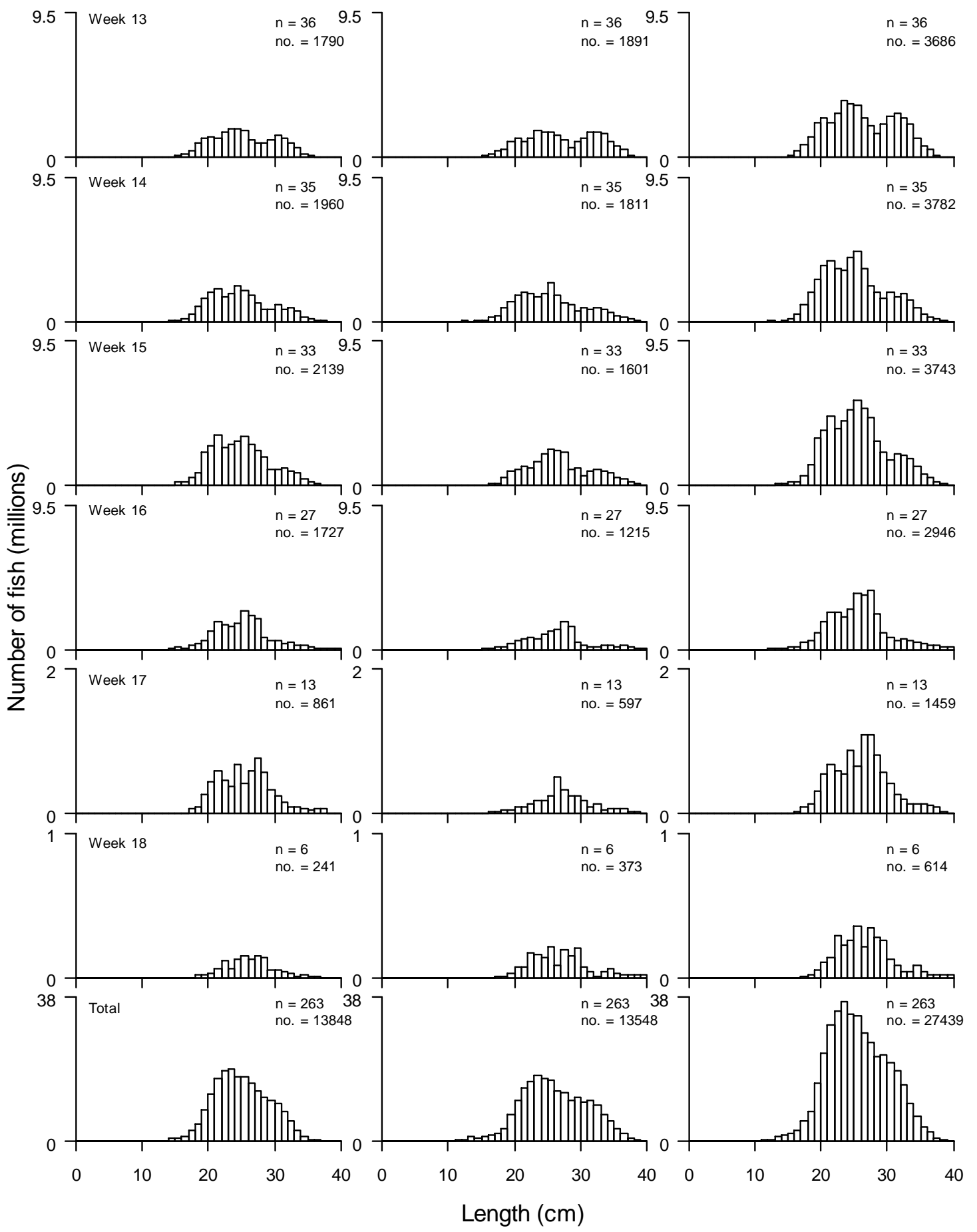

Figure A1e continued: AUCKLAND ISLANDS trawl fishery 2008 length frequency. 
Table A2a: Snares CPUE estimated values (arbitrary units) and CVs for the lognormal, target squid by week for the fishing years 1990, 1991 and 2008 (Hurst et al. 2012).

\begin{tabular}{|c|c|c|c|c|c|c|}
\hline & & 1990 & & 1991 & & 2008 \\
\hline & CPUE & $\mathrm{CV}$ & CPUE & $\mathrm{CV}$ & CPUE & $\mathrm{CV}$ \\
\hline 1 & 2.06 & 0.13 & 0.76 & 0.06 & - & - \\
\hline 2 & 2.72 & 0.12 & 0.61 & 0.06 & - & - \\
\hline 3 & 2.72 & 0.10 & 0.51 & 0.07 & - & - \\
\hline 4 & 1.62 & 0.12 & 0.63 & 0.06 & - & - \\
\hline 5 & 0.90 & 0.25 & 1.24 & 0.07 & 1.33 & 0.07 \\
\hline 6 & 1.12 & 0.15 & 1.64 & 0.06 & 0.66 & 0.06 \\
\hline 7 & 0.41 & 0.24 & 0.99 & 0.06 & 0.65 & 0.06 \\
\hline 8 & 1.24 & 0.24 & 0.99 & 0.06 & 1.29 & 0.06 \\
\hline 9 & 1.11 & 0.18 & 0.88 & 0.07 & 1.02 & 0.06 \\
\hline 10 & 1.30 & 0.23 & 1.14 & 0.07 & 1.01 & 0.07 \\
\hline 11 & 0.74 & 0.15 & 1.24 & 0.13 & 1.59 & 0.09 \\
\hline 12 & 0.68 & 0.16 & 2.44 & 0.06 & 1.25 & 0.17 \\
\hline 13 & 0.84 & 0.17 & 1.30 & 0.06 & 0.85 & 0.09 \\
\hline 14 & - & - & 0.93 & 0.08 & 0.67 & 0.10 \\
\hline 15 & 1.00 & 0.33 & 1.41 & 0.12 & 0.68 & 0.14 \\
\hline 16 & 0.53 & 0.43 & 0.63 & 0.20 & 0.80 & 0.15 \\
\hline 17 & 0.25 & 0.47 & 1.76 & 0.24 & 1.37 & 0.15 \\
\hline 18 & - & - & 1.14 & 0.18 & 1.95 & 0.35 \\
\hline 19 & - & - & 0.86 & 0.29 & & \\
\hline 20 & - & - & 0.60 & 0.36 & & \\
\hline 21 & - & - & CPUE & $\mathrm{CV}$ & & \\
\hline 22 & - & - & & & & \\
\hline
\end{tabular}

Table A2b: Auckland Islands CPUE estimated values (arbitrary units) and CVs for the lognormal, target squid by week for the fishing year 2008 (Hurst et al., 2012).

\begin{tabular}{rrr} 
& & 2008 \\
\cline { 2 - 3 } & CPUE & CV \\
1 & & \\
2 & - & - \\
3 & - & - \\
4 & - & - \\
5 & - & - \\
6 & - & - \\
7 & - & - \\
8 & 1.46 & 0.12 \\
9 & 1.24 & 0.09 \\
10 & 1.15 & 0.13 \\
11 & 1.14 & 0.08 \\
12 & 0.91 & 0.11 \\
13 & 1.53 & 0.11 \\
14 & 1.19 & 0.09 \\
15 & 0.89 & 0.08 \\
16 & 0.78 & 0.11 \\
17 & 0.37 & 0.16
\end{tabular}


Table A2c: Catch weights (t) for all vessels for each week for selected years (Hurst et al. 2012).

\begin{tabular}{rrrrrr} 
& & & \multicolumn{2}{c}{ Snares shelf } & Auckland Islands \\
Week & 1990 & 1991 & 1994 & 2008 & 2008 \\
1 & 784.7 & 555.0 & 0.00 & 1006.8 & - \\
2 & 1465.5 & 535.4 & - & 920.8 & - \\
3 & 1934.4 & 277.7 & - & 1750.6 & - \\
4 & 613.4 & 689.0 & - & 2208.6 & - \\
5 & 104.9 & 1056.8 & 0.07 & 2108.7 & 6.4 \\
6 & 227.7 & 2074.6 & 0.04 & 4154.9 & 6.5 \\
7 & 40.7 & 967.5 & 0.05 & 3205.3 & 89.3 \\
8 & 106.4 & 1278.0 & 0.08 & 2214.7 & 1327.5 \\
9 & 200.0 & 647.2 & 0.11 & 1360.0 & 2596.5 \\
10 & 140.2 & 793.7 & 0.06 & 2537.2 & 1032.6 \\
11 & 386.7 & 220.8 & 0.16 & 1689.4 & 2406.2 \\
12 & 172.2 & 3195.7 & 0.11 & 3209.5 & 1280.0 \\
13 & 245.3 & 1450.0 & 0.14 & 1295.1 & 1822.9 \\
14 & 6.2 & 433.3 & 0.12 & 731.2 & 1962.0 \\
15 & 41.0 & 207.7 & 0.30 & 230.9 & 2505.6 \\
16 & 19.5 & 41.5 & 0.15 & 265.0 & 1286.8 \\
17 & 12.7 & 89.7 & 0.14 & 102.5 & 325.2 \\
18 & - & 104.2 & 0.00 & 136.1 & 102.3 \\
19 & - & 70.8 & - & 50.5 & - \\
20 & - & - & 0.00 & - & - \\
21 & 3 & 0.00 & 0.18 & 3 & - \\
22 & 3 & 0.00 & 0.25 & 3 &
\end{tabular}




\section{Appendix B}

\section{Snares 2008}

Table B.1a: Calculated objective function and variance of standardised error of mean lengths for model fitted with 1, 2, and 3-cohorts and constant, linear and broken stick mean growth for Snares 2008.

\begin{tabular}{|c|ccc|ccc|}
\hline & \multicolumn{2}{|l|}{ Objective function } & \multicolumn{3}{l|}{ Variance of standardised error of } \\
mean lengths, $\operatorname{Var}\left(S_{l, w}\right)$
\end{tabular}

Table B.1b: Timing and size ofcohorts for models fitted with 1, 2, and 3-cohorts and constant, linear and broken stick mean growth for Snares 2008.

\begin{tabular}{|l|rr|r|}
\hline 1-cohort & \multicolumn{2}{|c|}{ Cohort 1 } & \multicolumn{1}{|c|}{ Total size } \\
\cline { 1 - 2 } $\begin{array}{l}\text { Number of } \\
\text { parameters }\end{array}$ & week & \multicolumn{1}{c|}{ size* } & \\
\hline 1 & 0.9 & 213 & 213 \\
2 & 1.4 & 2,741 & 2,741 \\
3 & 1.3 & 2,615 & 2,615 \\
\hline
\end{tabular}

\begin{tabular}{|c|c|c|c|c|c|}
\hline 2-cohorts & \multicolumn{2}{|c|}{ Cohort 1} & \multicolumn{2}{|c|}{ Cohort 2} & \multirow[b]{2}{*}{ Total size } \\
\hline $\begin{array}{l}\text { Number of } \\
\text { parameters }\end{array}$ & week & size* & week & size* & \\
\hline 1 & -8.0 & 2,741 & 4.3 & 1,957 & 4,698 \\
\hline 2 & -7.8 & 2,466 & 4.9 & 1,889 & 4,355 \\
\hline 3 & -7.8 & 2,729 & 5.0 & 2,056 & 4,785 \\
\hline
\end{tabular}

\begin{tabular}{|c|c|c|c|c|c|c|c|}
\hline 3-cohorts & \multicolumn{2}{|c|}{ Cohort 1} & \multicolumn{2}{|c|}{ Cohort 2} & \multicolumn{2}{|c|}{ Cohort 3} & \multirow[b]{2}{*}{ Total size } \\
\hline $\begin{array}{l}\text { Number of } \\
\text { parameters }\end{array}$ & week & size $*$ & week & $\operatorname{size}^{*}$ & week & size $*$ & \\
\hline 1 & -8.3 & 2,465 & 4.3 & 975 & 5.0 & 655 & 4,095 \\
\hline 2 & -6.6 & 2,248 & 4.1 & 1,537 & 7.0 & 508 & 4.293 \\
\hline 3 & -5.0 & 2,741 & 4.9 & 1,599 & 5.2 & 1,124 & 5,464 \\
\hline
\end{tabular}

*numbers in millions

Table B.1c: Estimated parameters for Snares 2008 models with 1, 2, and 3-cohorts and constant, linear and broken stick mean growth for Snares 2008.

\begin{tabular}{|l|r|rrrrrr|}
\hline $\begin{array}{l}\text { Number of } \\
\text { cohorts }\end{array}$ & Mean growth & \multicolumn{2}{l}{$\begin{array}{l}\mu_{\text {sel }} \\
(\mathrm{cm})\end{array}$} & $\sigma_{\text {selL }}$ & $\sigma_{\text {selR }}$ & $\begin{array}{l}\mu_{\text {recruit }} \\
(\mathrm{cm})\end{array}$ & \multicolumn{2}{l|}{$\begin{array}{l}\text { Escapeme } \\
\text { nt }\end{array}$} \\
\hline \multirow{3}{*}{1} & $\begin{array}{r}\text { constant } \\
\text { linear }\end{array}$ & 25.02 & 6.62 & 2.80 & 9.07 & 8.59 & 0.85 \\
& 22.00 & 4.97 & 3.61 & 18.75 & 3.60 & 0.95 \\
& broken stick & 22.30 & 5.16 & 3.46 & 13.37 & 6.63 & 0.94 \\
\hline \multirow{3}{*}{2} & constant & 22.00 & 5.61 & 3.60 & 15.82 & 2.96 & 0.89 \\
& linear & 21.62 & 4.46 & 3.58 & 13.45 & 3.53 & 0.89 \\
& broken stick & 21.61 & 4.47 & 3.58 & 13.47 & 3.53 & 0.90 \\
\hline \multirow{3}{*}{3} & constant & 21.91 & 5.80 & 3.59 & 16.52 & 3.08 & 0.88 \\
& linear & 21.57 & 4.68 & 3.57 & 13.16 & 3.64 & 0.90 \\
& broken stick & 21.46 & 5.06 & 3.76 & 17.91 & 2.58 & 0.92 \\
\hline
\end{tabular}




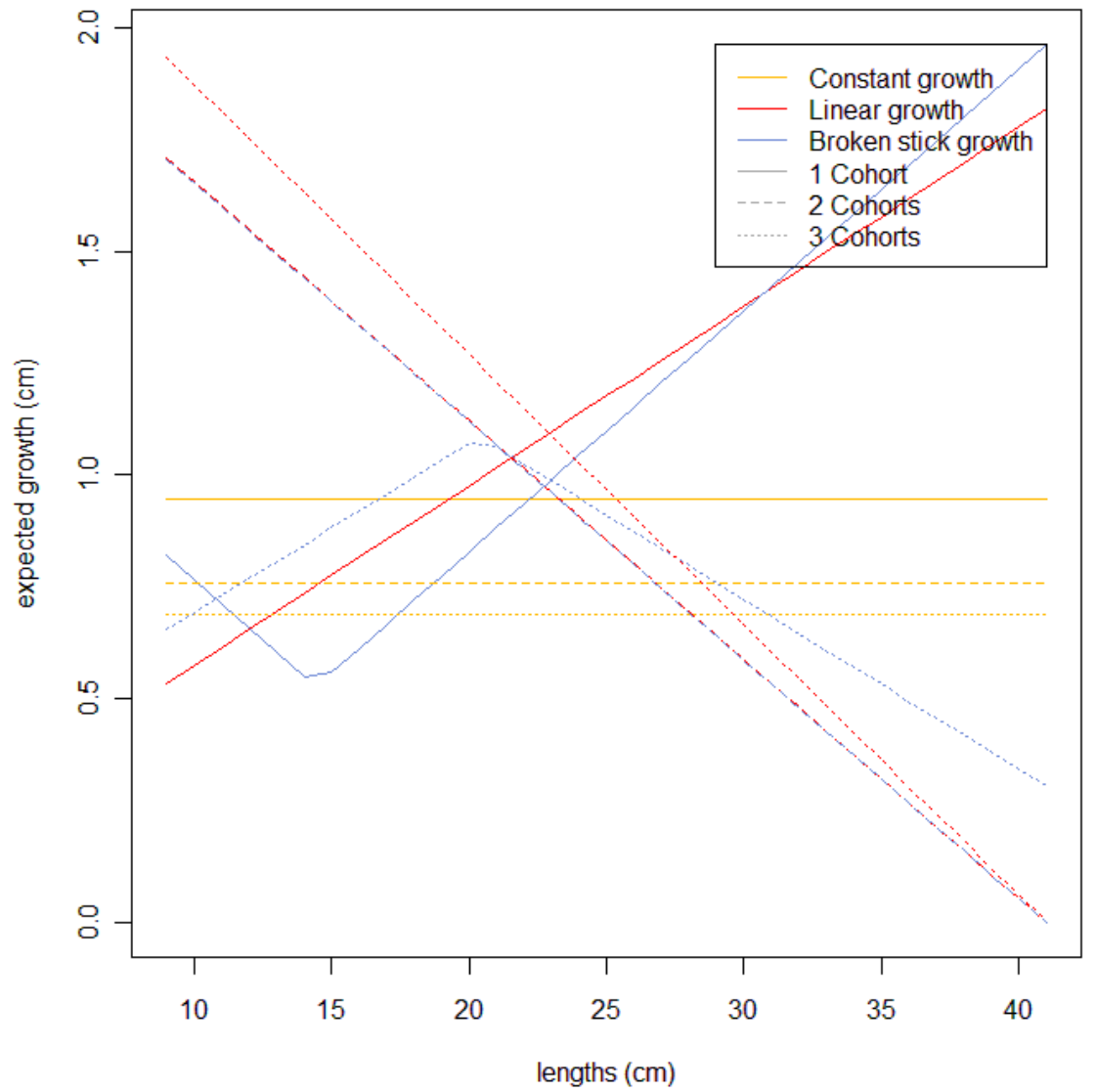

Figure B.1a: Expected mean growth per week by length from models with 1, 2, and 3-cohorts and constant, linear and broken stick mean growth for Snares 2008. The colour of each line indicates the mean growth function and the type of line indicates the number of cohorts. 

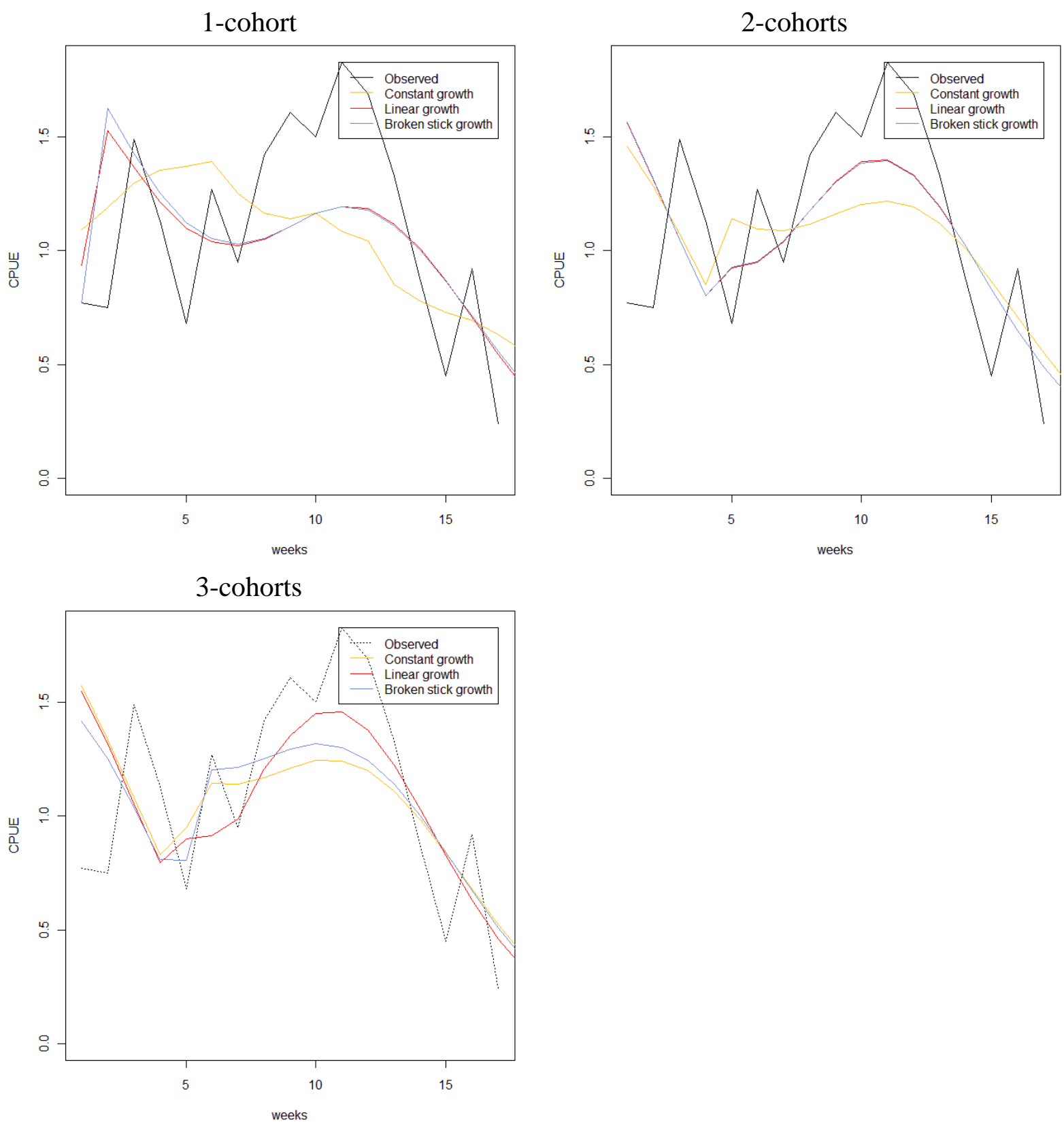

Figure B.1b: Observed CPUE compared to expected CPUE for models with 1, 2, and 3-cohorts and constant, linear and broken stick mean growth for Snares 2008. 
1-cohort

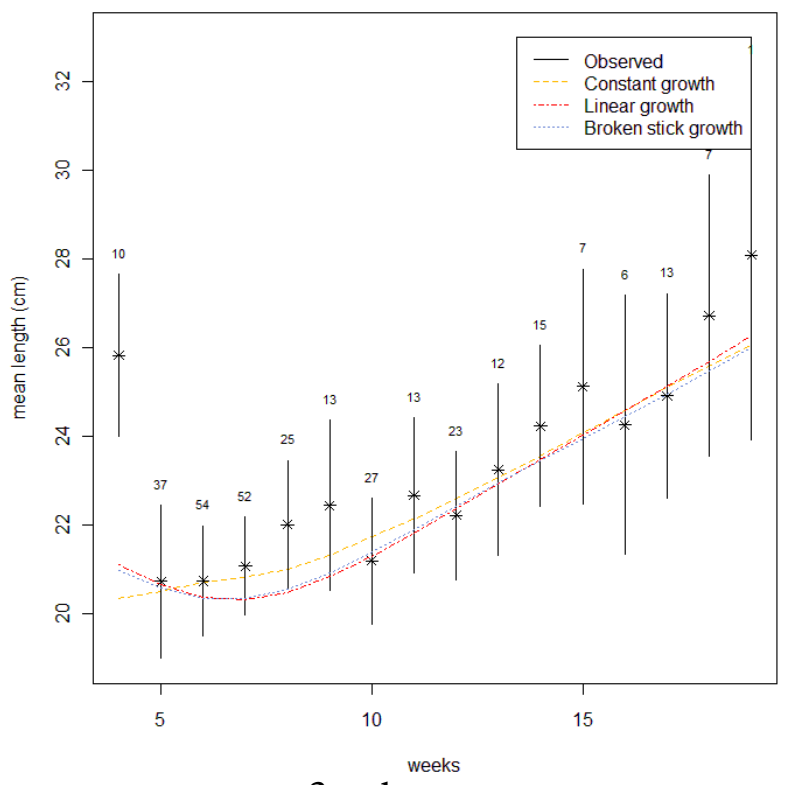

3-cohorts

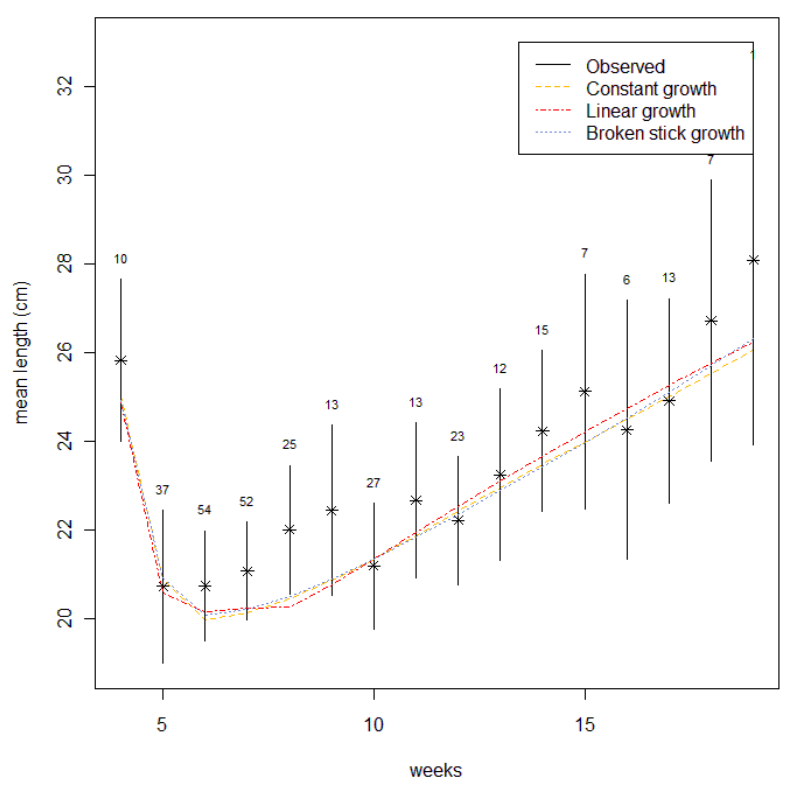

2-cohorts

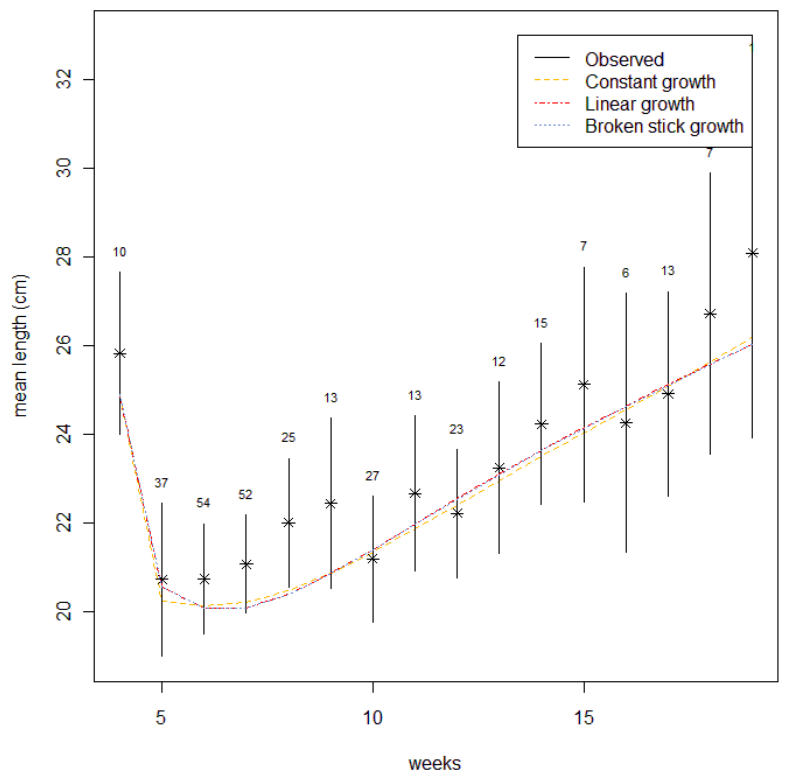

Figure B.1c: Observed mean lengths with $95 \%$ confidence intervals, compared to expected mean lengths for models with 1, 2 and 3-cohorts and constant, linear and broken stick mean growth for Snares 2008. The vertical black lines show the $95 \%$ confidence intervals for the observations, calculated using the number of tows (the numbers above the black lines) as the actual sample sizes. The asterisks are the mean lengths from the data. 
1-cohort
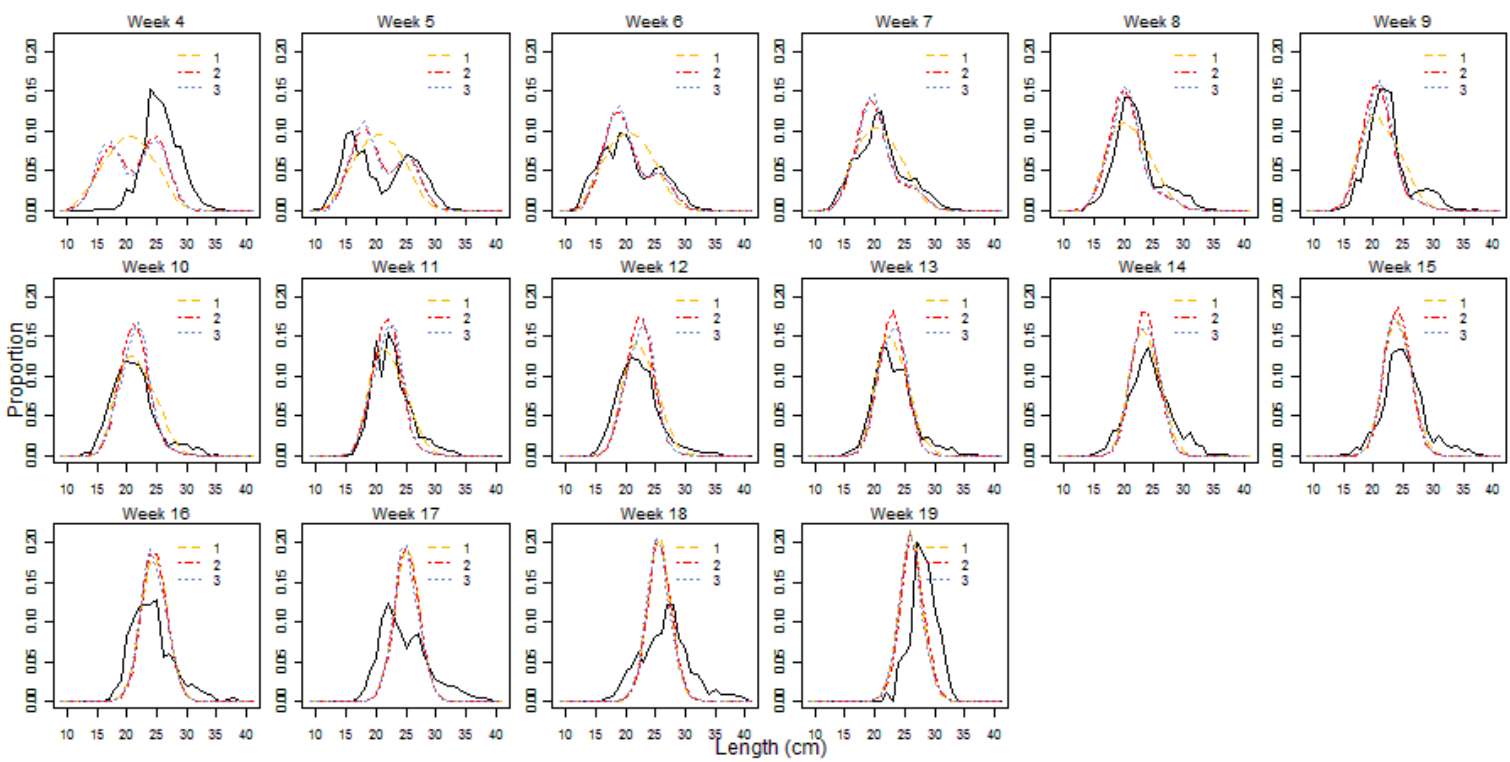

2-cohorts
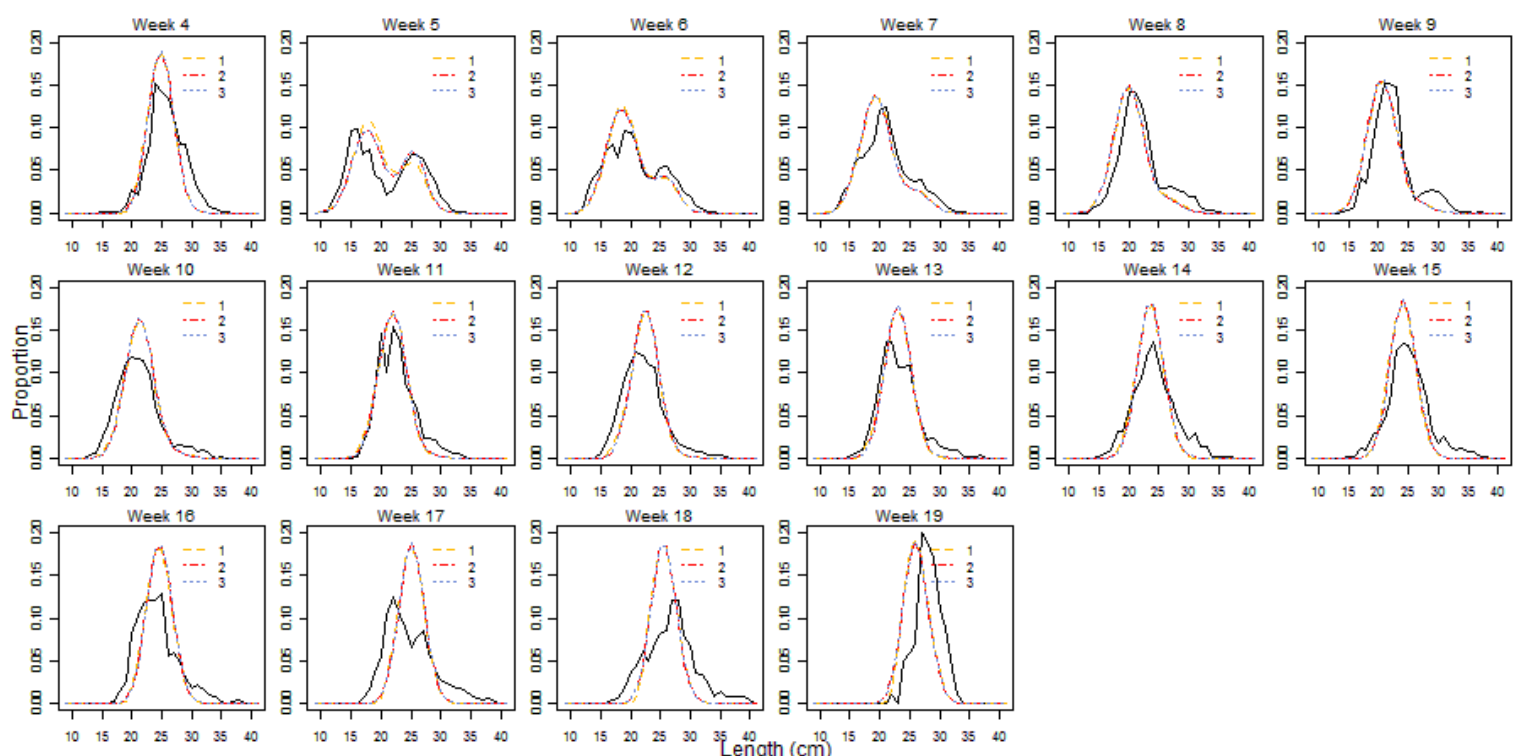

3-cohorts
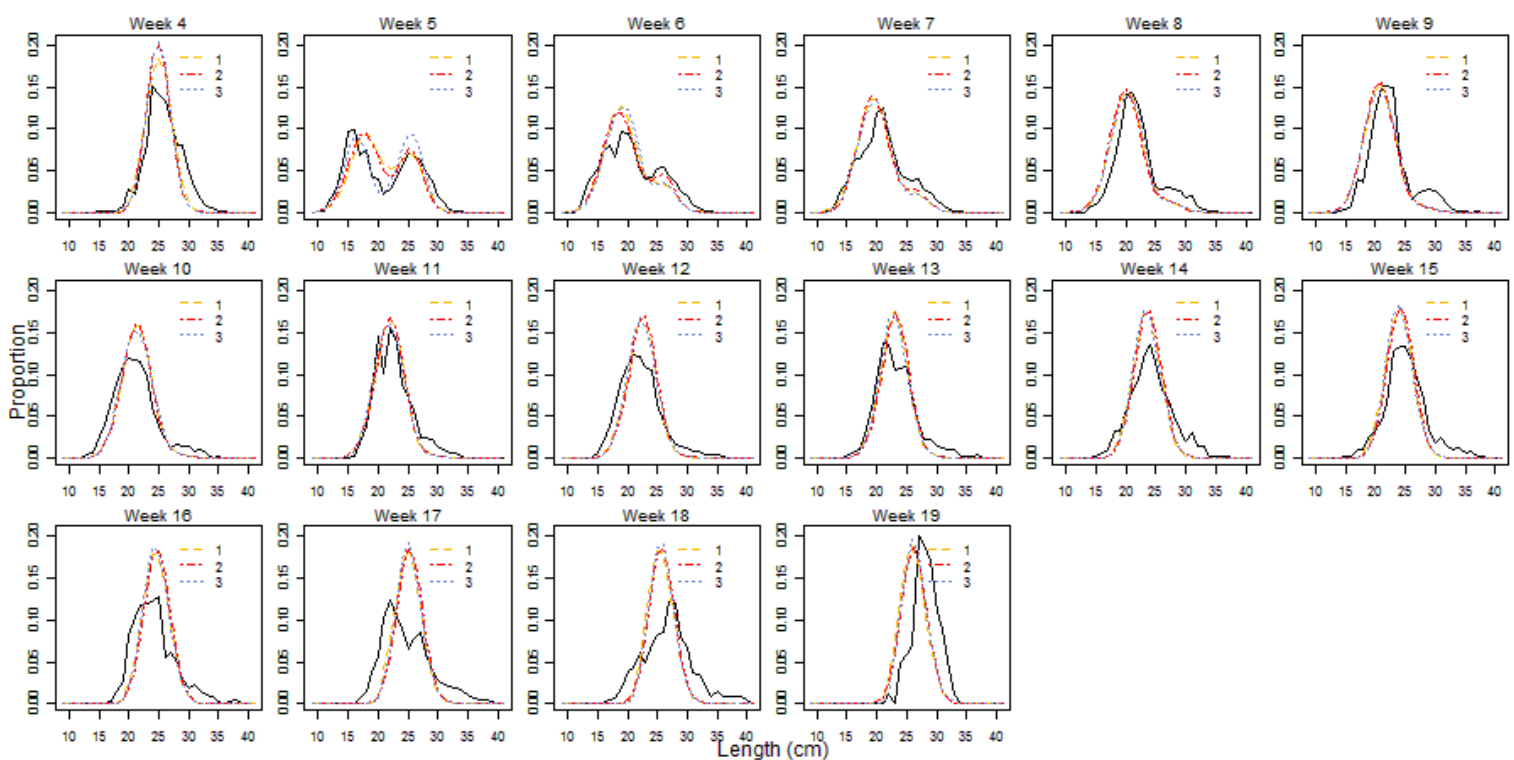

Figure B.1d: Observed length frequencies compared to expected length frequencies for models with 1, 2 and 3-cohorts and constant, linear and broken stick mean growth for Snares 2008. 
Snares and Auckland Islands 2008

Table B.2a: Calculated objective function and variance of standardised error of mean lengths for model fitted with 1, 2, and 3-cohorts and constant, linear and broken stick mean growth for Snares and Auckland Islands 2008.

\begin{tabular}{|c|ccc|ccc|}
\hline & \multicolumn{2}{|l|}{ Objective function } & \multicolumn{3}{l|}{ Variance of standardised error of } \\
mean lengths, $\operatorname{Var}\left(S_{l, w}\right)$
\end{tabular}

Table B.2b: Timing and size of cohorts for models fitted with 1, 2, and 3-cohorts and constant, linear and broken stick mean growth for Snares and Auckland Islands 2008.

\begin{tabular}{|c|cc|c|}
\hline 1-cohort & \multicolumn{2}{|c|}{ Cohort 1 } & \multirow{2}{*}{ Total size } \\
pumber of & week & size* & \\
\hline 1 & -6.5 & 152 & 152 \\
2 & -3.5 & 122 & 122 \\
3 & -9.2 & 156 & 156 \\
\hline
\end{tabular}

\begin{tabular}{|c|cc|cc|c|}
\hline 2-cohorts & \multicolumn{2}{|c|}{ Cohort 1 } & \multicolumn{2}{c|}{ Cohort 2 } & \multirow{2}{*}{ Total size } \\
\cline { 1 - 4 } $\begin{array}{c}\text { Number of } \\
\text { parameters }\end{array}$ & week & size* & week & size $*$ & \\
\hline 1 & -8.6 & 22 & 4.0 & 86 & 108 \\
2 & -7.7 & 25 & 2.2 & 95 & 120 \\
3 & -3.6 & 108 & 9.4 & 41 & 149 \\
\hline
\end{tabular}

\begin{tabular}{|c|c|c|c|c|c|c|c|}
\hline 3-cohorts & \multicolumn{2}{|c|}{ Cohort 1} & \multicolumn{2}{|c|}{ Cohort 2} & \multicolumn{2}{|c|}{ Cohort 3} & \multirow[b]{2}{*}{ Total size } \\
\hline $\begin{array}{l}\text { Number of } \\
\text { parameters }\end{array}$ & week & size * & week & size * & week & size * & \\
\hline 1 & -4.8 & 21 & 4.0 & 60 & 10.0 & 24 & 105 \\
\hline 2 & -4.8 & 21 & 4.0 & 60 & 10.0 & 24 & 105 \\
\hline 3 & -6.0 & 22 & 4.0 & 40 & 4.9 & 53 & 115 \\
\hline
\end{tabular}

*numbers in millions

Table B.2c: Expected parameters for Snares and Auckland Islands 2008 models with constant, linear and broken stick mean growth and 1, 2, and 3-cohorts.

\begin{tabular}{|c|c|c|c|c|c|c|c|}
\hline $\begin{array}{l}\text { Number of } \\
\text { cohorts }\end{array}$ & $\begin{array}{l}\text { Number of } \\
\text { mean growth } \\
\text { parameters }\end{array}$ & $\begin{array}{l}\mu_{\text {sel }} \\
(\mathrm{cm})\end{array}$ & $\sigma_{\text {selL }}$ & $\sigma_{\text {SelR }}$ & $\begin{array}{l}\mu_{\text {recruit }} \\
(\mathrm{cm})\end{array}$ & $\sigma_{\text {recruit }}$ & $\begin{array}{l}\text { Escapeme } \\
\mathrm{nt}\end{array}$ \\
\hline \multirow{3}{*}{1} & 1 & 38.63 & 7.66 & 0.50 & 15.93 & 5.00 & 0.39 \\
\hline & 2 & 37.99 & 7.51 & 0.61 & 17.68 & 4.82 & 0.58 \\
\hline & 3 & 37.69 & 6.93 & 0.70 & 14.47 & 4.29 & 0.27 \\
\hline \multirow{3}{*}{2} & 1 & 35.84 & 6.56 & 1.23 & 17.99 & 4.92 & 0.63 \\
\hline & 2 & 38.43 & 7.69 & 0.50 & 16.94 & 3.31 & 0.49 \\
\hline & 3 & 36.78 & 6.61 & 1.02 & 16.32 & 4.96 & 0.57 \\
\hline \multirow{3}{*}{3} & 1 & 34.61 & 6.49 & 1.82 & 17.74 & 4.70 & 0.84 \\
\hline & 2 & 34.61 & 6.49 & 1.82 & 17.74 & 4.70 & 0.84 \\
\hline & 3 & 36.58 & 7.35 & 8.95 & 17.64 & 3.89 & 0.60 \\
\hline
\end{tabular}




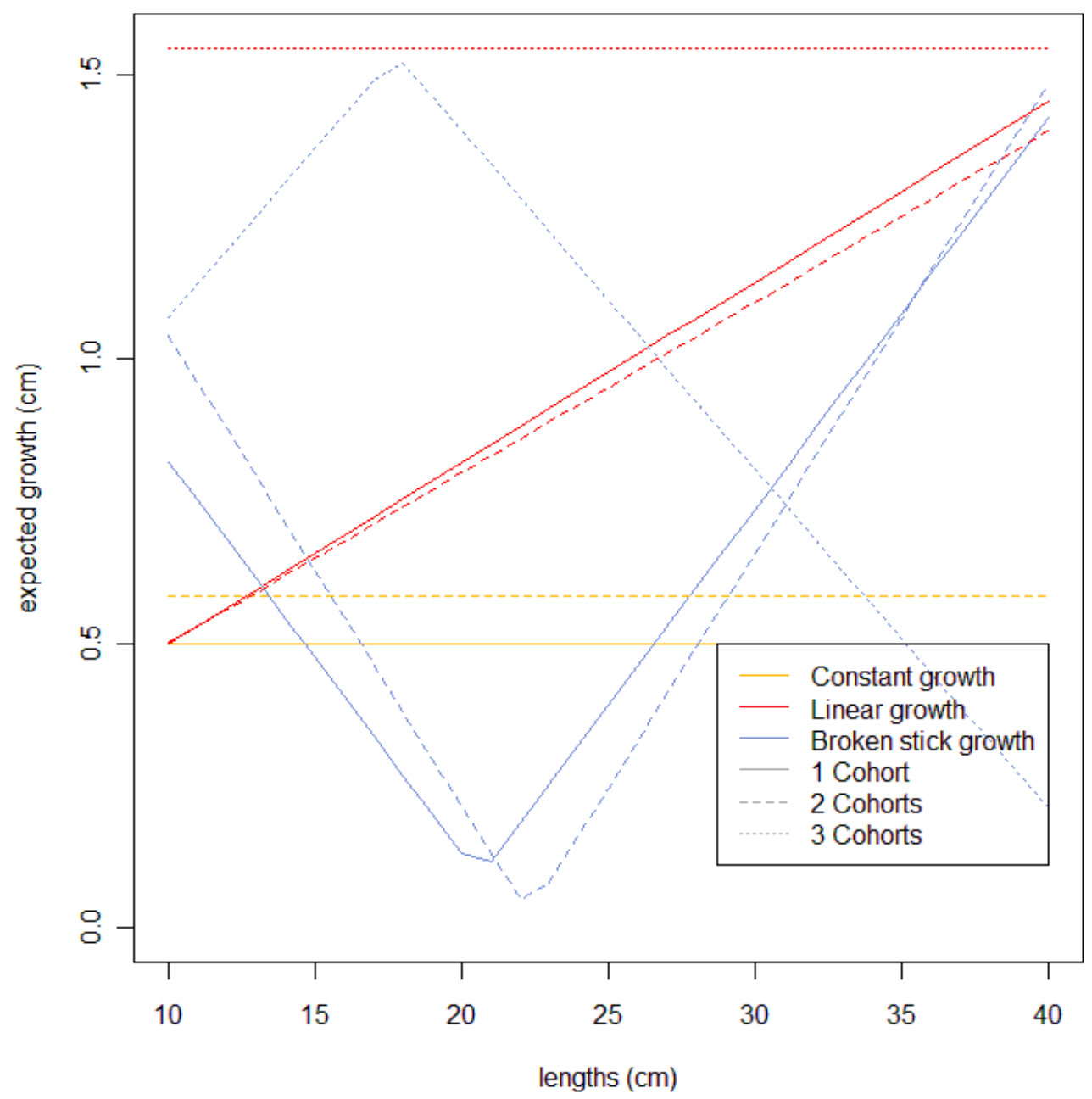

Figure B.2a: Expected mean growth by length for Snares and Auckland Islands 2008 from models with constant, linear and broken stick mean growth and 1, 2 and 3-cohorts. Mean growth is the mean growth for 1 week. 

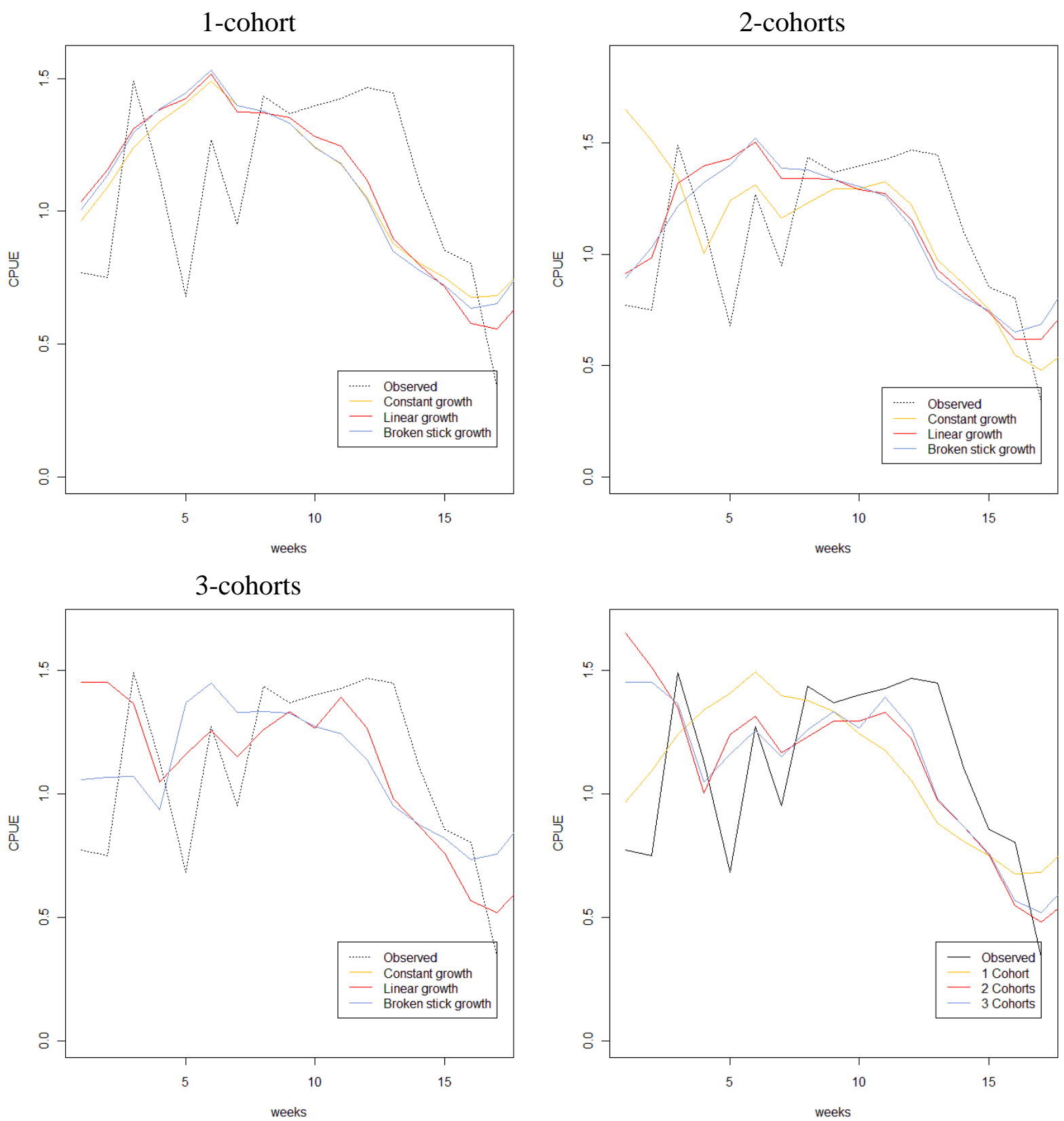

Figure B.2b: Observed CPUE compared to expected $q \times$ exploitable biomass for Snares and Auckland Islands 2008, for constant, linear and broken stick mean growth and 1, 2 and 3-cohorts. 
1 -cohort

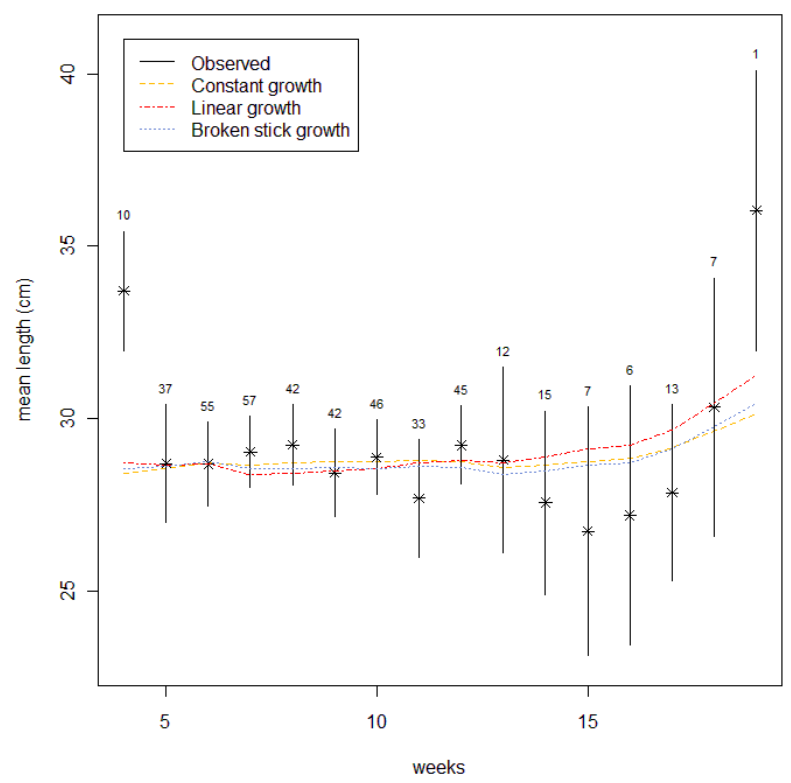

3-cohorts

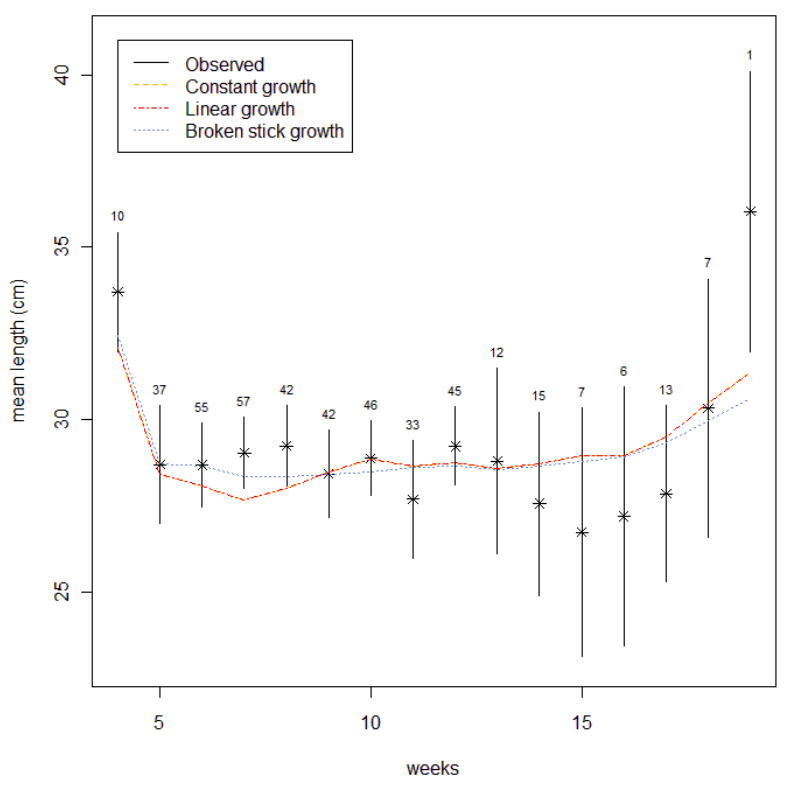

2-cohorts
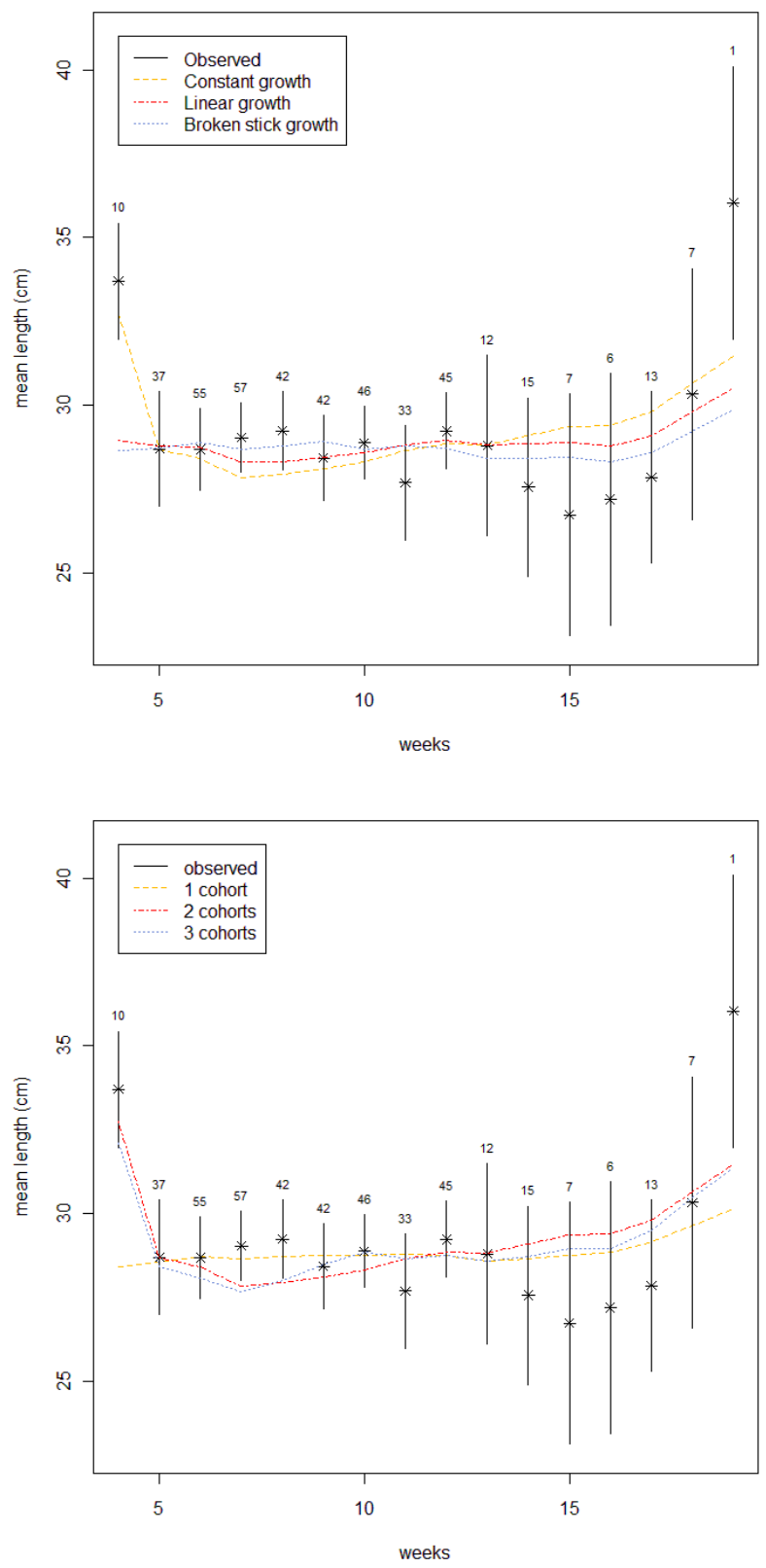

Figure B.2c: Observed mean lengths with $95 \%$ confidence intervals, compared to expected mean lengths for Snares and Auckland Islands 2008, for constant, linear and broken stick mean growth and 1, 2 and 3cohorts. The vertical black lines show the $95 \%$ confidence intervals for the observations, calculated using the number of tows (the numbers above the black lines) as the actual sample sizes. 
1-cohort
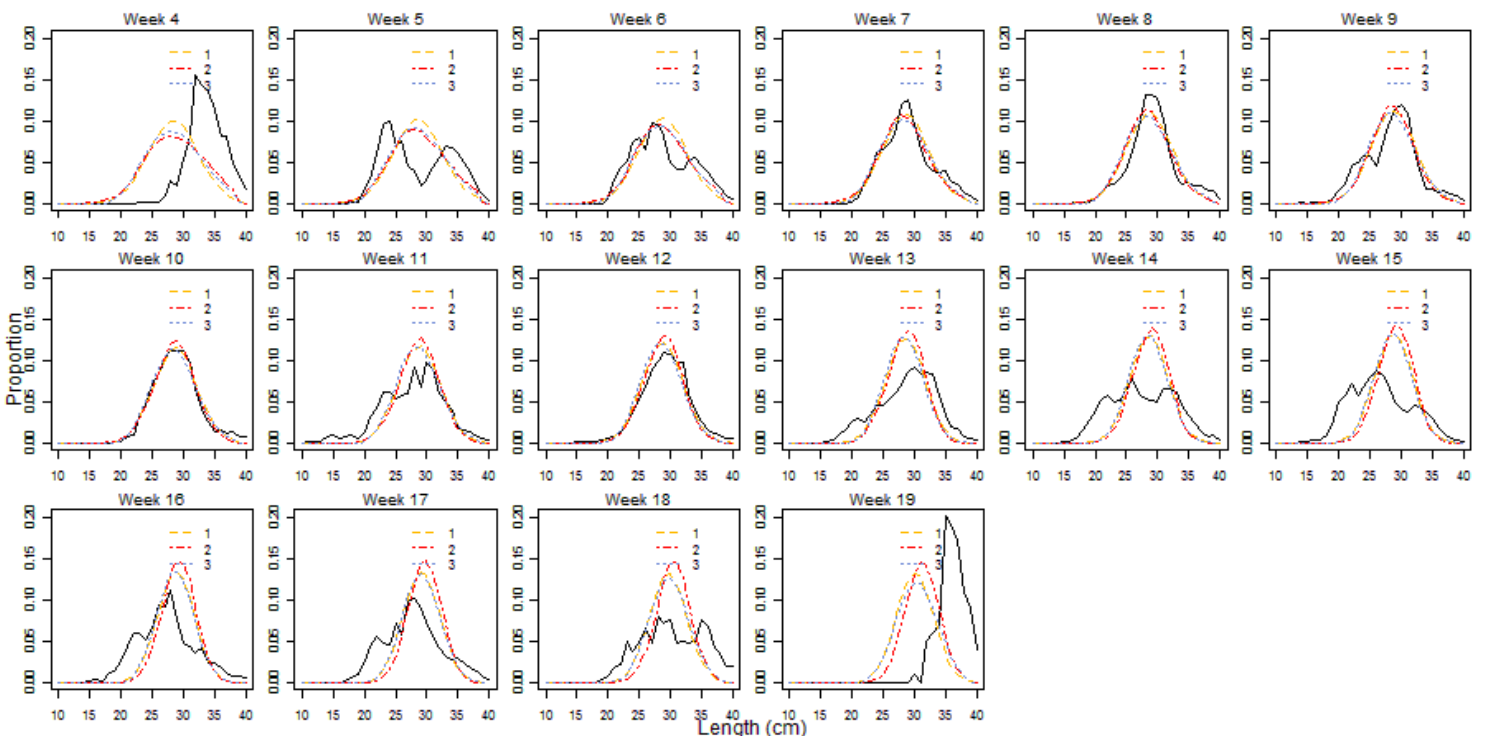

2-cohorts
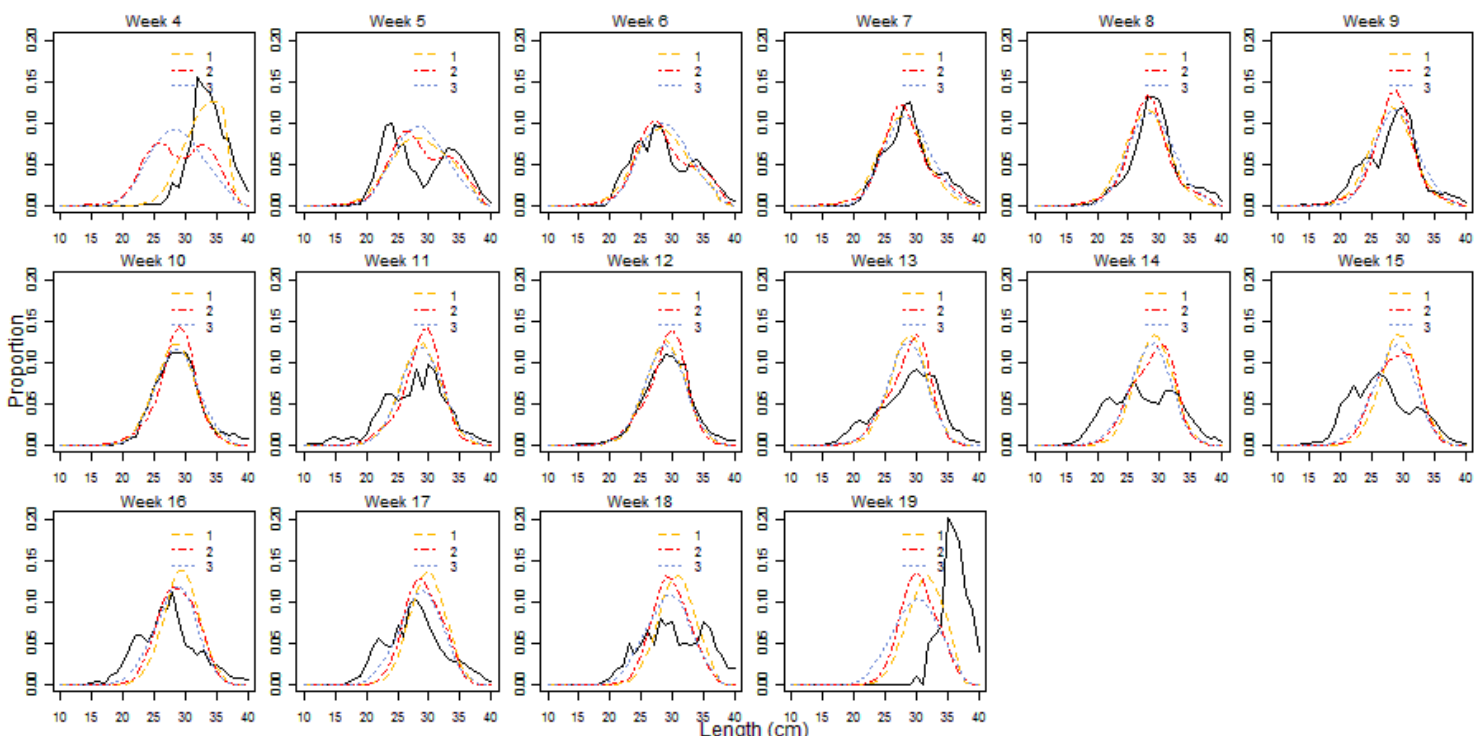

3-cohorts
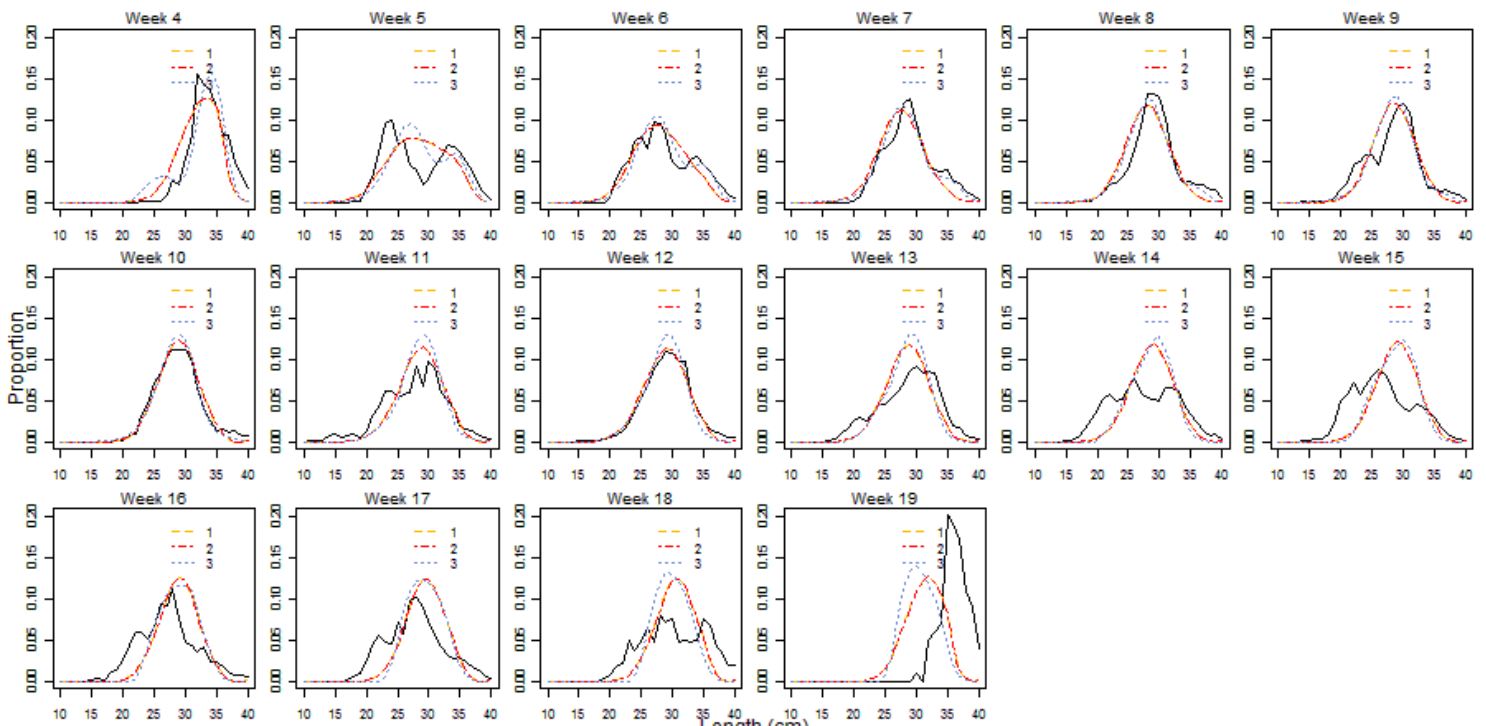

Figure B.2d: Observed length frequencies compared to expected length frequencies for Snares and Auckland Islands 2008, for constant, linear and broken stick mean growth and 1, 2 and 3-cohorts. The last set of graphs show the constant growth (1 mean growth parameter) model with 1,2 and 3-cohorts. 


\section{Constant growth}
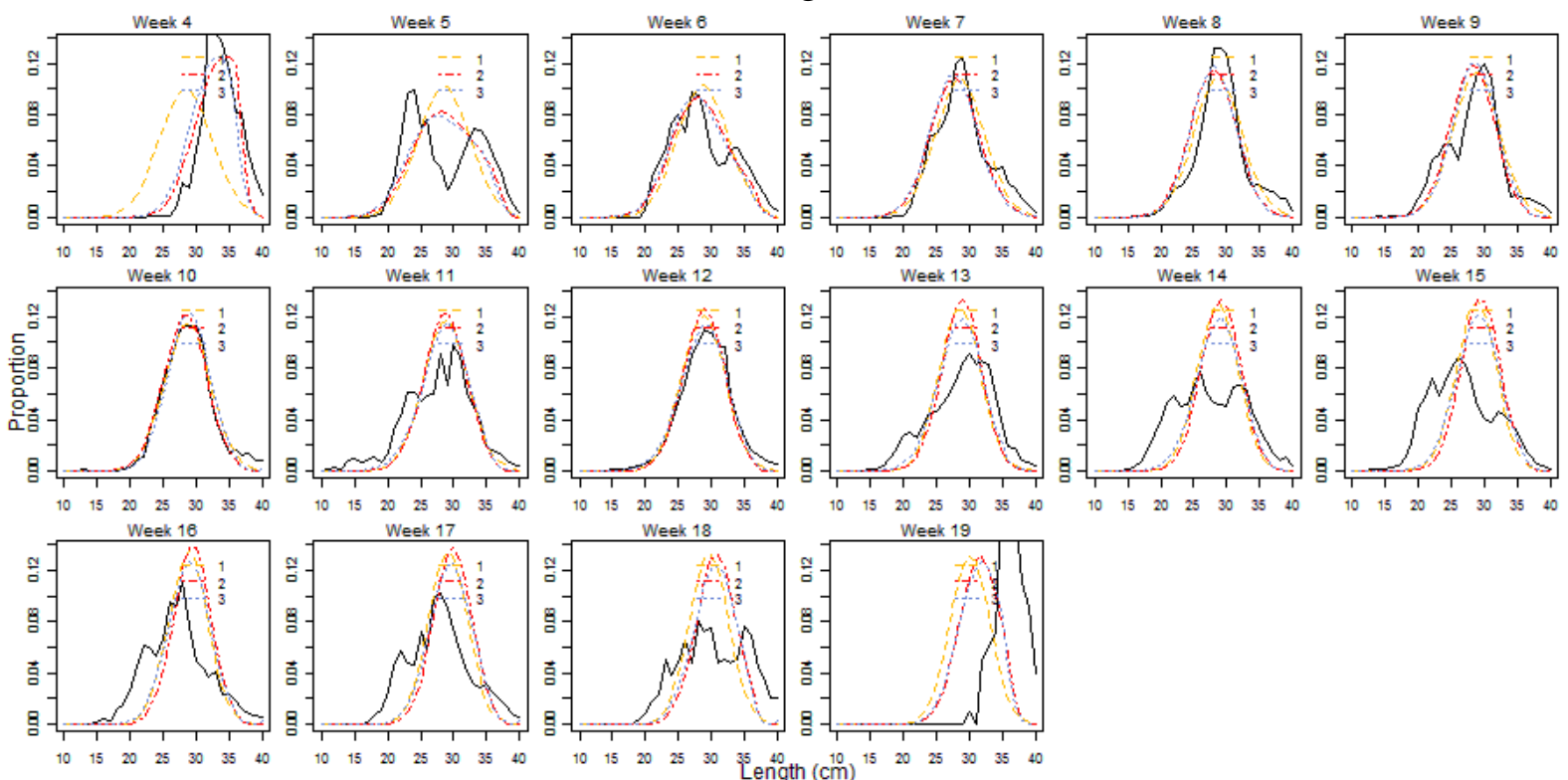

Figure B.2d: (continued). 
Snares 1991

Table B.3a: The calculated objective function and variance of the standardised error of mean lengths for models fitted with 1, 2, and 3-cohorts and constant, linear and broken stick mean growth for Snares 1991.

\begin{tabular}{|c|ccc|ccc|}
\hline & \multicolumn{2}{|l|}{ Objective function } & \multicolumn{3}{|l|}{ Variance of standardised error of } \\
mean lengths, $\operatorname{Var}\left(S_{l, w}\right)$
\end{tabular}

Table B.3b: Timing and size of cohorts for models fitted with 1, 2, and 3-cohorts and constant, linear and broken stick mean growth for Snares 1991.

\begin{tabular}{|l|rr|r|}
\hline 1-cohort & \multicolumn{2}{|c|}{ Cohort 1 } & \multirow{2}{*}{ Total size } \\
\cline { 1 - 2 } pumber of & week & \multicolumn{1}{c|}{ size* } & \\
\hline 1 & & & 238 \\
2 & -3.0 & 243 & 243 \\
3 & -1.0 & 171 & 171 \\
\hline
\end{tabular}

\begin{tabular}{|c|c|c|c|c|c|}
\hline 2-cohorts & \multicolumn{2}{|c|}{ Cohort 1} & \multicolumn{2}{|c|}{ Cohort 2} & \multirow[b]{2}{*}{ Total size } \\
\hline $\begin{array}{l}\text { Number of } \\
\text { parameters }\end{array}$ & week & size* & week & size* & \\
\hline 1 & -2.3 & 1,332 & 12.0 & 732 & 2,064 \\
\hline 2 & -1.3 & 2,057 & 12.0 & 1,142 & 3,199 \\
\hline 3 & 0.7 & 590 & 14.0 & 280 & 870 \\
\hline
\end{tabular}

\begin{tabular}{|c|c|c|c|c|c|c|c|}
\hline 3-cohorts & \multicolumn{2}{|c|}{ Cohort 1} & \multicolumn{2}{|c|}{ Cohort 2} & \multicolumn{2}{|c|}{ Cohort 3} & \multirow[b]{2}{*}{ Total size } \\
\hline $\begin{array}{l}\text { Number of } \\
\text { parameters }\end{array}$ & week & Population* & week & size* $^{*}$ & week & size* & \\
\hline 1 & 0.7 & 764 & 4.6 & 1,102 & 13.0 & 955 & 2,821 \\
\hline 2 & 0.7 & 49 & 4.9 & 77 & 13.0 & 33 & 159 \\
\hline 3 & 0.7 & 49 & 4.9 & 77 & 13.0 & 33 & 159 \\
\hline
\end{tabular}

*numbers in millions

Table B.3c: Expected parameters for Snares 1991 models with constant, linear and broken stick mean growth and 1, 2, and 3-cohorts.

\begin{tabular}{|c|c|c|c|c|c|c|c|}
\hline $\begin{array}{l}\text { Number of } \\
\text { cohorts }\end{array}$ & $\begin{array}{l}\text { Number of } \\
\text { mean growth } \\
\text { parameters }\end{array}$ & $\begin{array}{l}\mu_{\text {sel }} \\
(\mathrm{cm})\end{array}$ & $\sigma_{\text {selL }}$ & $\sigma_{\text {selR }}$ & $\begin{array}{l}\mu_{\text {recruit }} \\
(\mathrm{cm})\end{array}$ & $\sigma_{\text {recruit }}$ & $\begin{array}{l}\text { Escapeme } \\
\mathrm{nt}\end{array}$ \\
\hline \multirow{3}{*}{1} & 1 & 25.45 & 14.13 & 0.50 & 9.00 & 2.51 & 0.74 \\
\hline & 2 & 25.33 & 19.95 & 0.50 & 9.00 & 3.19 & 0.54 \\
\hline & 3 & 25.33 & 7.07 & 0.50 & 13.02 & 0.73 & 0.70 \\
\hline \multirow{3}{*}{2} & 1 & 14.61 & 1.05 & 6.47 & 9.45 & 3.29 & 0.91 \\
\hline & 2 & 14.46 & 0.99 & 6.58 & 9.52 & 3.64 & 0.91 \\
\hline & 3 & 14.89 & 1.34 & 6.42 & 11.77 & 2.81 & 0.86 \\
\hline \multirow{3}{*}{3} & 1 & 14.25 & 5.78 & 6.20 & 15.28 & 1.18 & 0.95 \\
\hline & 2 & 24.18 & 20.00 & 1.27 & 15.01 & 1.25 & 0.41 \\
\hline & 3 & 24.18 & 20.00 & 1.27 & 15.01 & 1.25 & 0.40 \\
\hline
\end{tabular}




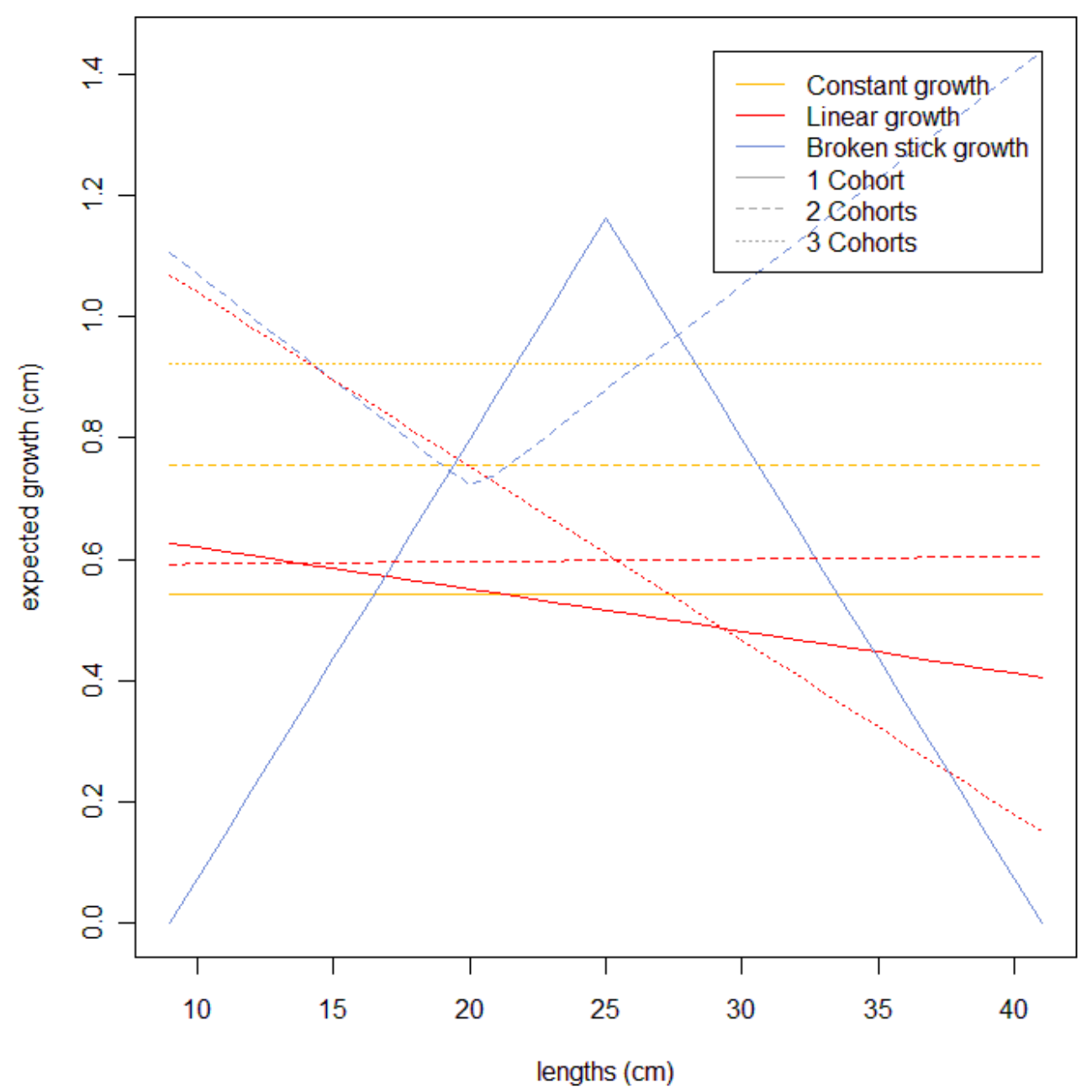

Figure B.3a: Expected mean growth by length for Snares 1991 from models with constant, linear and broken stick mean growth and 1, 2, and 3-cohorts. Mean growth is the mean growth for 1 week. 
1-cohort

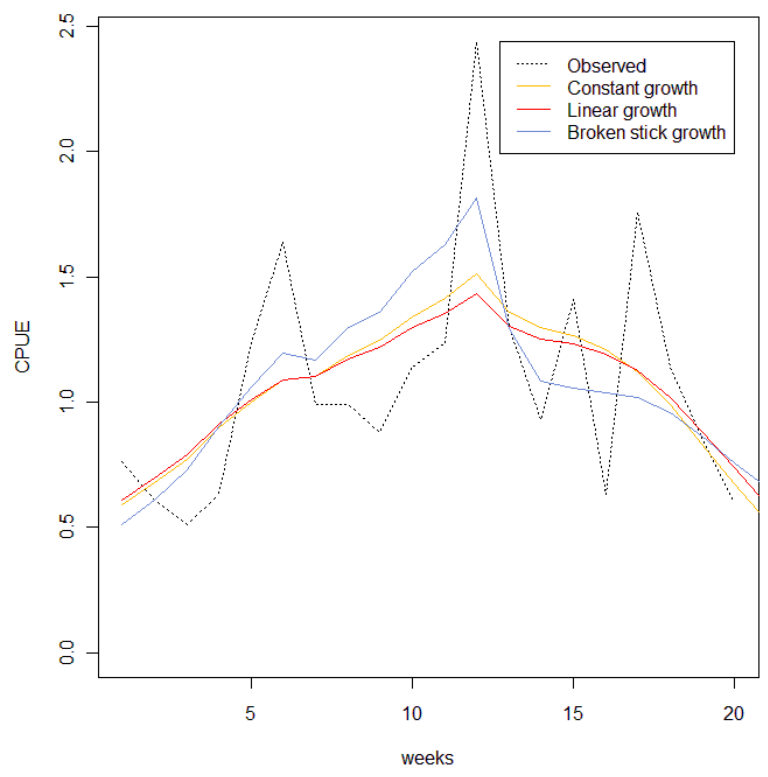

3-cohorts

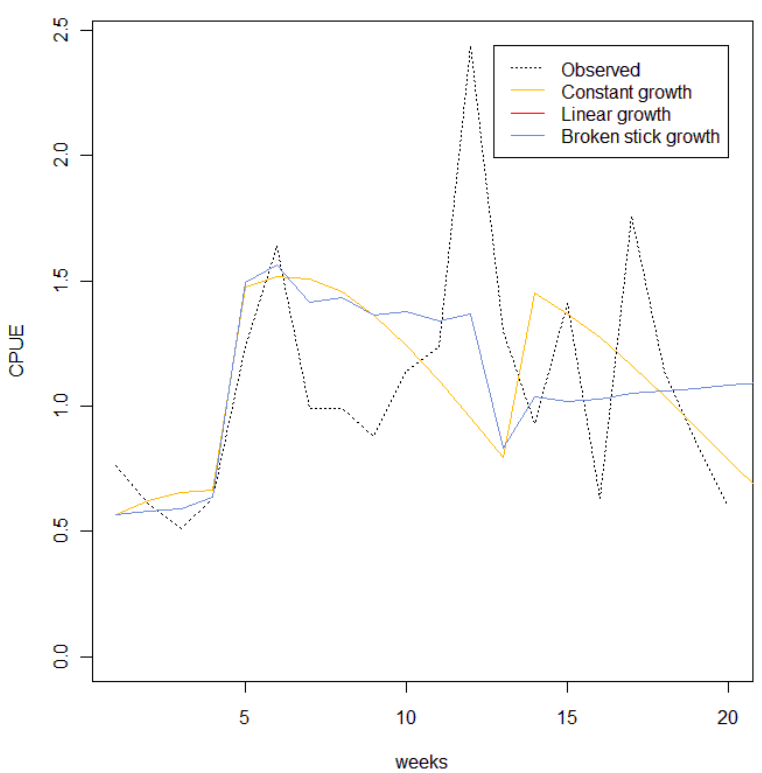

2-cohorts

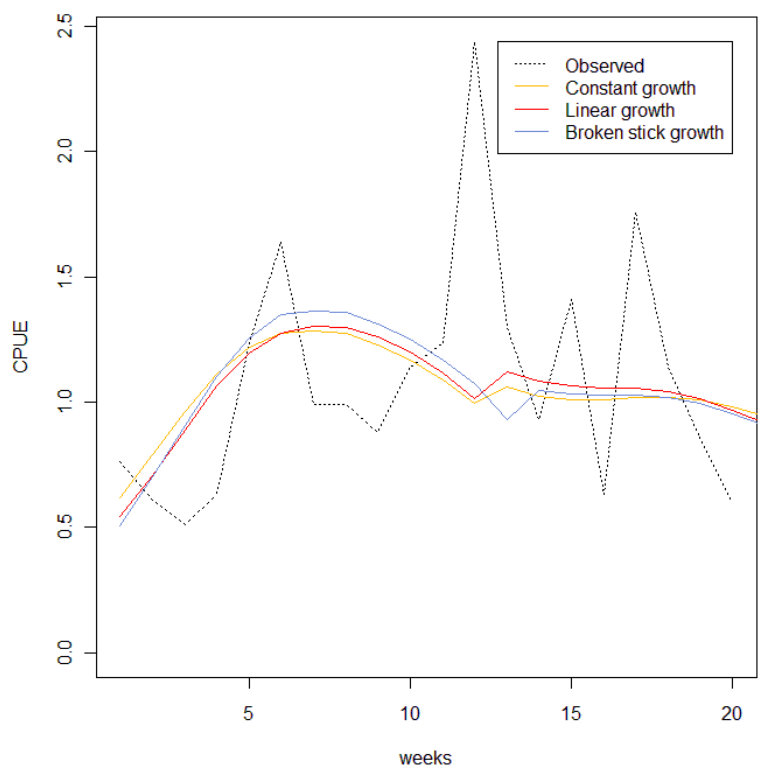

Figure B.3b: Observed CPUE compared to expected q $\times$ exploitable biomass for Snares 1991, for constant, linear and broken stick mean growth and 1, 2 and 3-cohorts. 
1-cohort

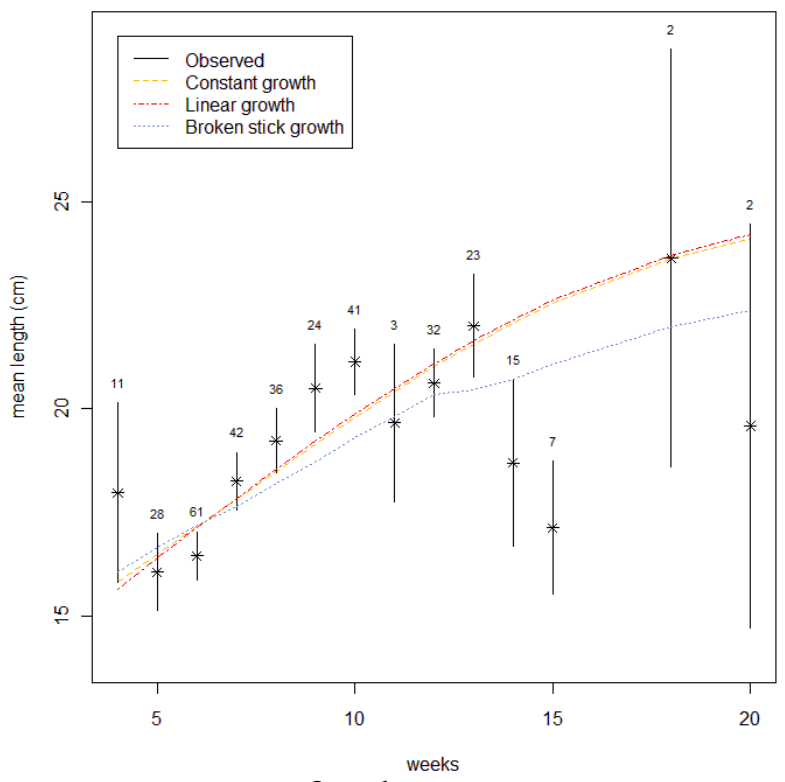

3-cohorts

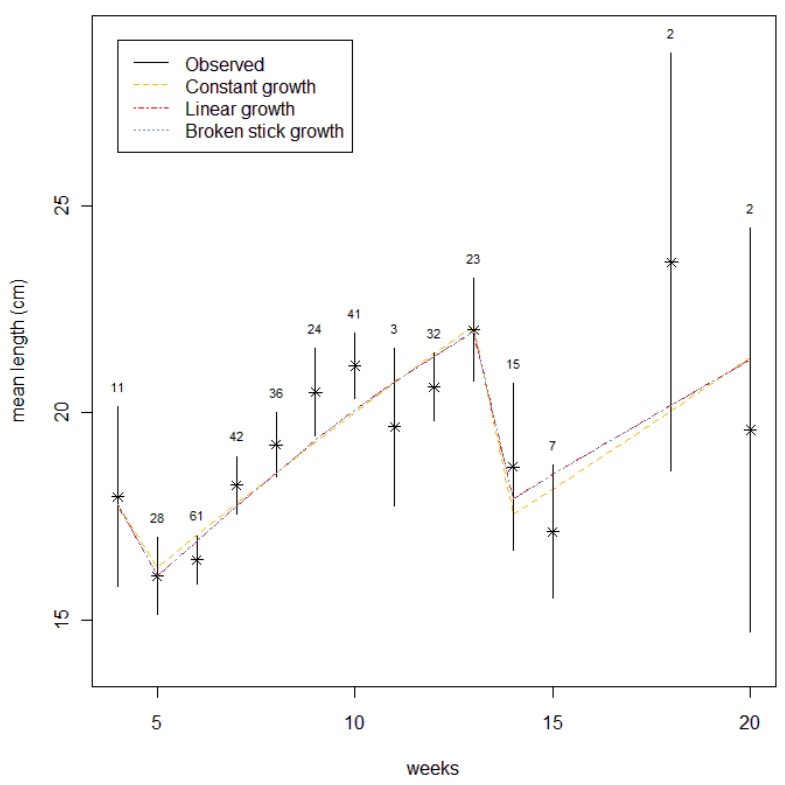

2-cohorts

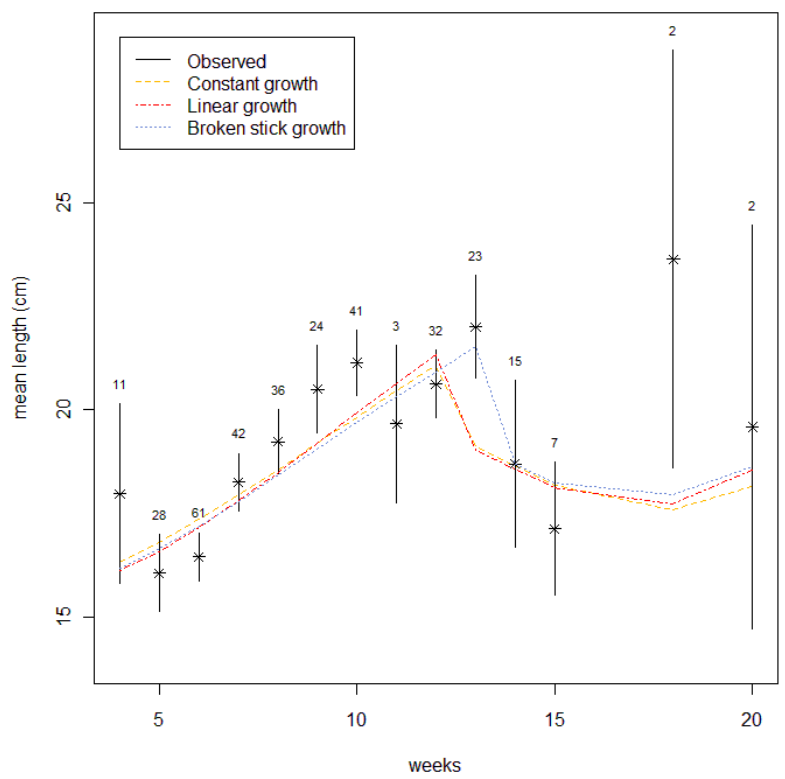

Figure B.3c: Observed mean lengths with $95 \%$ confidence intervals, compared to expected mean lengths for Snares 1991, for constant, linear and broken stick mean growth and 1, 2 and 3-cohorts. The vertical black lines show the $95 \%$ confidence intervals for the observations, calculated using the number of tows (the numbers above the black lines) as the actual sample sizes. 
1-cohort
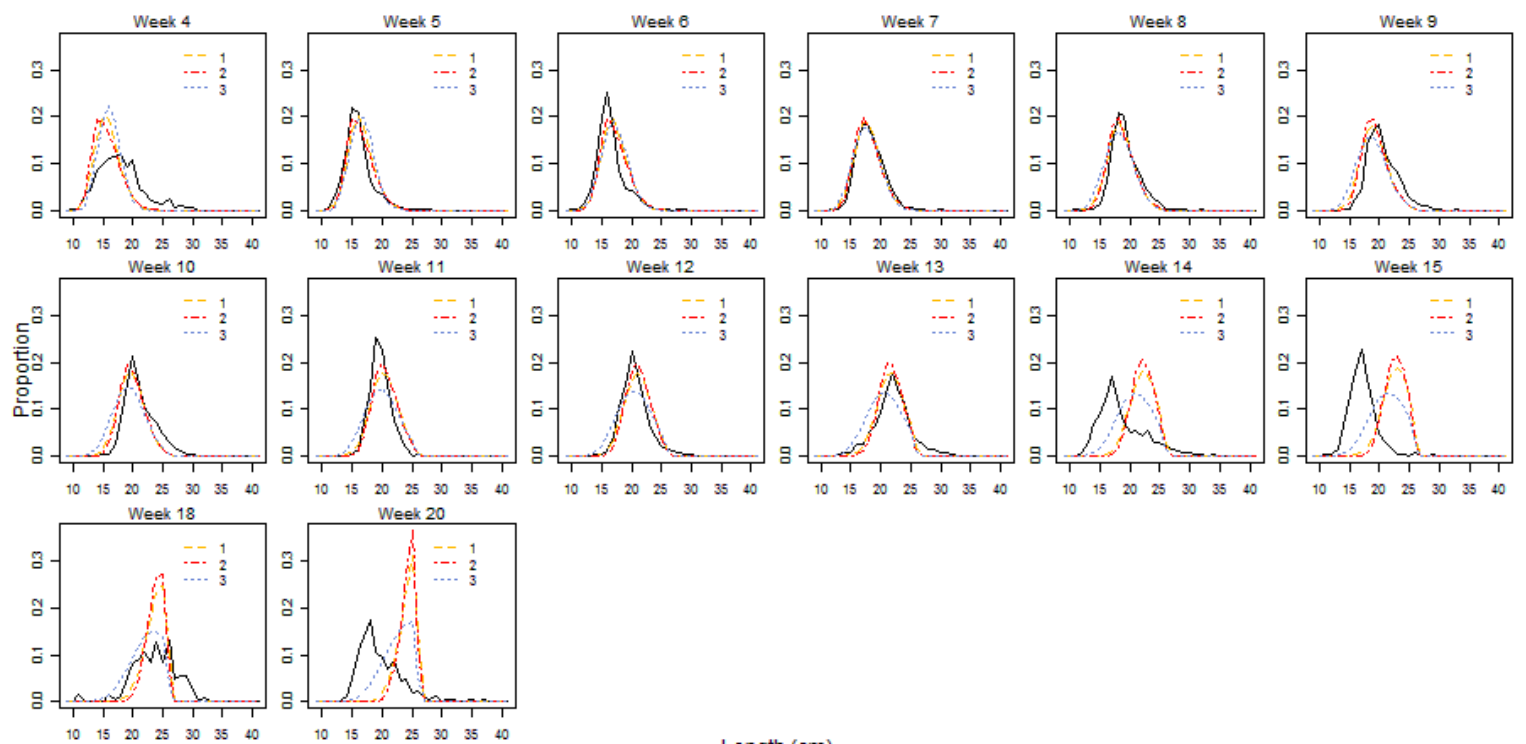

Length $(\mathrm{cm})$

2-cohorts
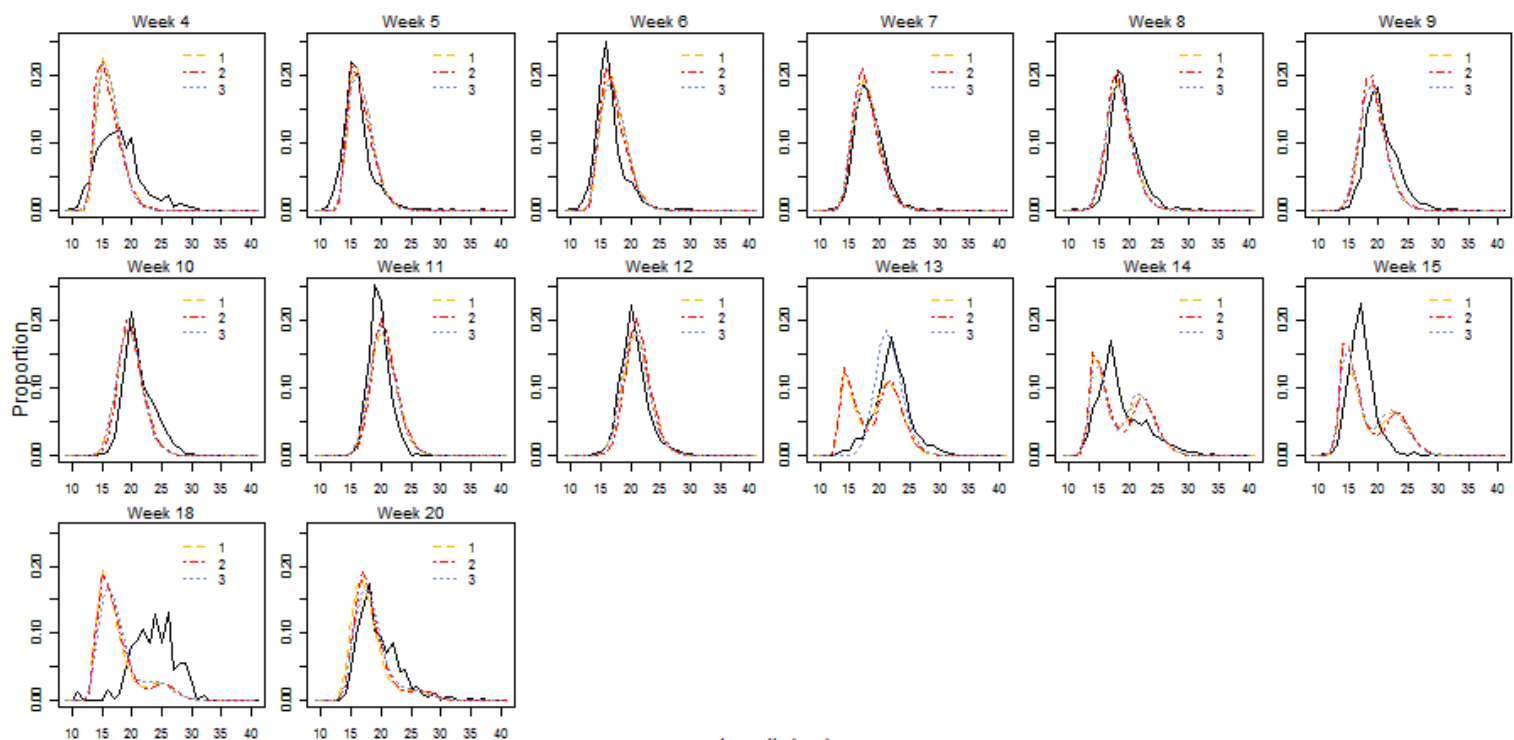

Length $(\mathrm{cm})$

3-cohorts
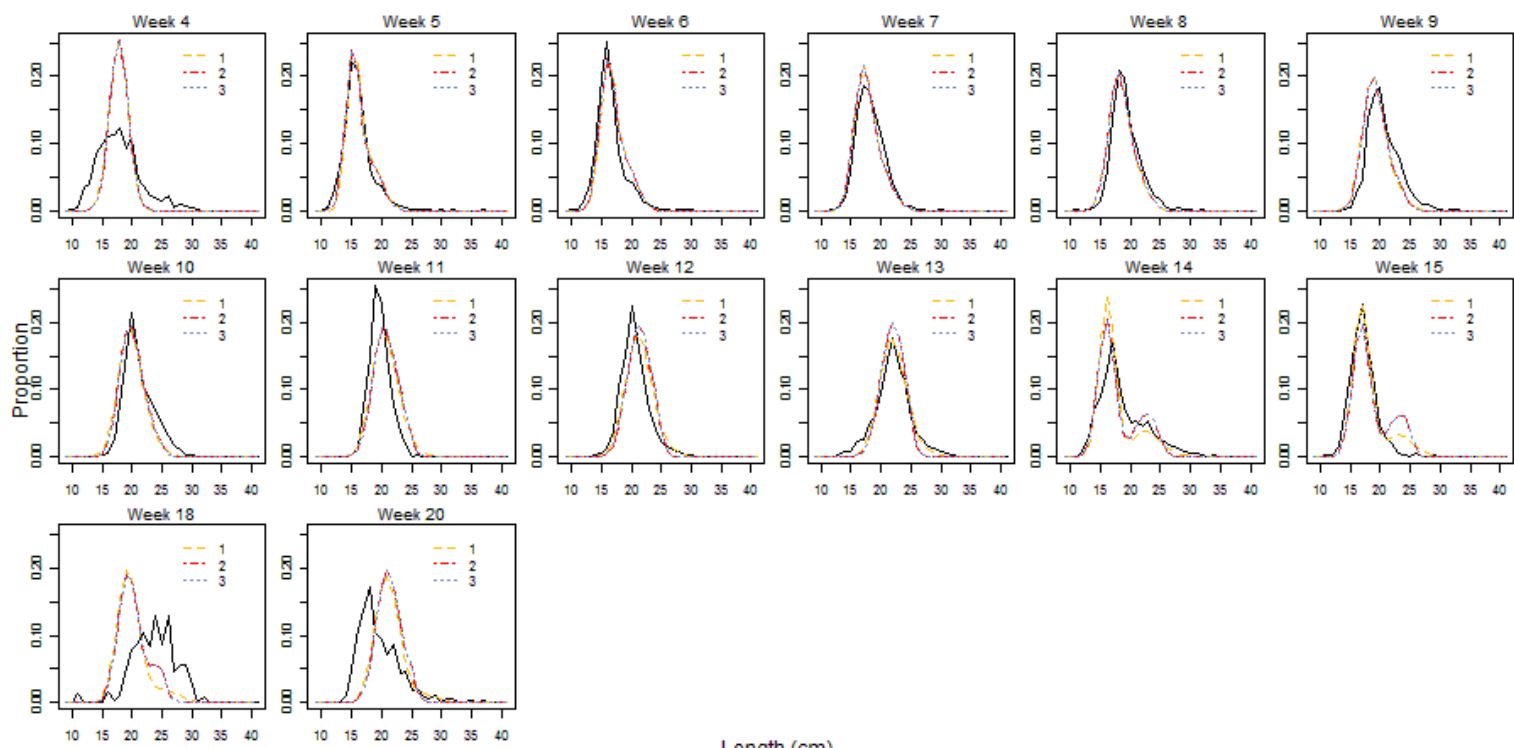

Length $(\mathrm{cm})$

Figure B.3d: Observed length frequencies compared to expected length frequencies for Snares 1991, for constant, linear and broken stick mean growth and 1, 2 and 3-cohorts. 
Snares 1990

Table B.4a: The calculated objective function and the variance of the standardised error of mean lengths for model fitted with 1, 2 and 3-cohorts and constant, linear and broken stick mean growth for Snares 1990.

\begin{tabular}{|c|ccc|ccc|}
\hline & \multicolumn{2}{|l|}{ Objective function } & \multicolumn{4}{l|}{ Variance of standardised error of } \\
mean lengths, $\operatorname{Var}\left(S_{l, w}\right)$
\end{tabular}

Table B.4b: Timing and size of cohorts for models fitted with 1, 2, and 3-cohorts and constant, linear and broken stick mean growth for Snares 1990.

\begin{tabular}{|l|lr|r|}
\hline 1-cohort & \multicolumn{2}{|c|}{ Cohort 1 } & \multirow{2}{*}{ Total size } \\
\cline { 1 - 2 } Number of & week & size* & \\
\hline 1 & & 55 & 55 \\
2 & -10.0 & 47 & 47 \\
3 & -5.8 & 47 & 47 \\
\hline
\end{tabular}

\begin{tabular}{|c|c|c|c|c|c|}
\hline 2-cohorts & \multicolumn{2}{|c|}{ Cohort 1} & \multicolumn{2}{|c|}{ Cohort 2} & \multirow[b]{2}{*}{ Total size } \\
\hline $\begin{array}{l}\text { Number of } \\
\text { parameters }\end{array}$ & week & $\operatorname{size}^{*}$ & week & $\operatorname{size}^{*}$ & \\
\hline 1 & -2.0 & 40 & 7.4 & 15 & 55 \\
\hline 2 & 0.0 & 34 & 7.9 & 10 & 44 \\
\hline 3 & -1.1 & 35 & 7.1 & 11 & 46 \\
\hline
\end{tabular}

\begin{tabular}{|c|c|c|c|c|c|c|c|}
\hline 3-cohorts & \multicolumn{2}{|c|}{ Cohort 1} & \multicolumn{2}{|c|}{ Cohort 2} & \multicolumn{2}{|c|}{ Cohort 3} & \multirow[b]{2}{*}{ Total size } \\
\hline $\begin{array}{l}\text { Number of } \\
\text { parameters }\end{array}$ & week & $\operatorname{size}^{*}$ & week & $\operatorname{size}^{*}$ & week & $\operatorname{size}^{*}$ & \\
\hline$\frac{1}{1}$ & -2.0 & 40 & 7.4 & 15 & 12.9 & 0 & 55 \\
\hline 2 & 0.0 & 34 & 7.9 & 10 & 12.0 & 0 & 44 \\
\hline 3 & -1.1 & 27 & 1.9 & 6 & 7.1 & 11 & 44 \\
\hline
\end{tabular}

*numbers in millions

Table B.4c: Expected parameters for Snares 1990 models with constant, linear and broken stick mean growth and 1, 2, and 3-cohorts.

\begin{tabular}{|c|c|c|c|c|c|c|c|}
\hline $\begin{array}{l}\text { Number of } \\
\text { cohorts }\end{array}$ & $\begin{array}{l}\text { Number of } \\
\text { mean growth } \\
\text { parameters }\end{array}$ & $\begin{array}{l}\mu_{\text {sel }} \\
(\mathrm{cm})\end{array}$ & $\sigma_{\text {selL }}$ & $\sigma_{\text {selR }}$ & $\begin{array}{l}\mu_{\text {recruit }} \\
(\mathrm{cm})\end{array}$ & $\sigma_{\text {recruit }}$ & $\begin{array}{l}\text { Escapeme } \\
\mathrm{nt}\end{array}$ \\
\hline \multirow{3}{*}{1} & 1 & 23.36 & 4.22 & 1.21 & 9.00 & 4.23 & 0.51 \\
\hline & 2 & 24.27 & 5.60 & 0.50 & 12.10 & 5.00 & 0.69 \\
\hline & 3 & 24.27 & 5.59 & 0.50 & 12.09 & 5.00 & 0.75 \\
\hline \multirow{3}{*}{2} & 1 & 24.06 & 12.14 & 0.80 & 16.04 & 2.09 & 0.83 \\
\hline & 2 & 24.80 & 11.26 & 0.50 & 18.00 & 2.02 & 0.77 \\
\hline & 3 & 24.83 & 10.49 & 0.59 & 17.30 & 1.76 & 0.79 \\
\hline \multirow{3}{*}{3} & 1 & 24.06 & 13.20 & 0.81 & 16.01 & 2.04 & 0.48 \\
\hline & 2 & 24.80 & 11.26 & 0.50 & 18.00 & 2.02 & 0.77 \\
\hline & 3 & 24.83 & 19.70 & 0.83 & 17.46 & 1.48 & 0.93 \\
\hline
\end{tabular}




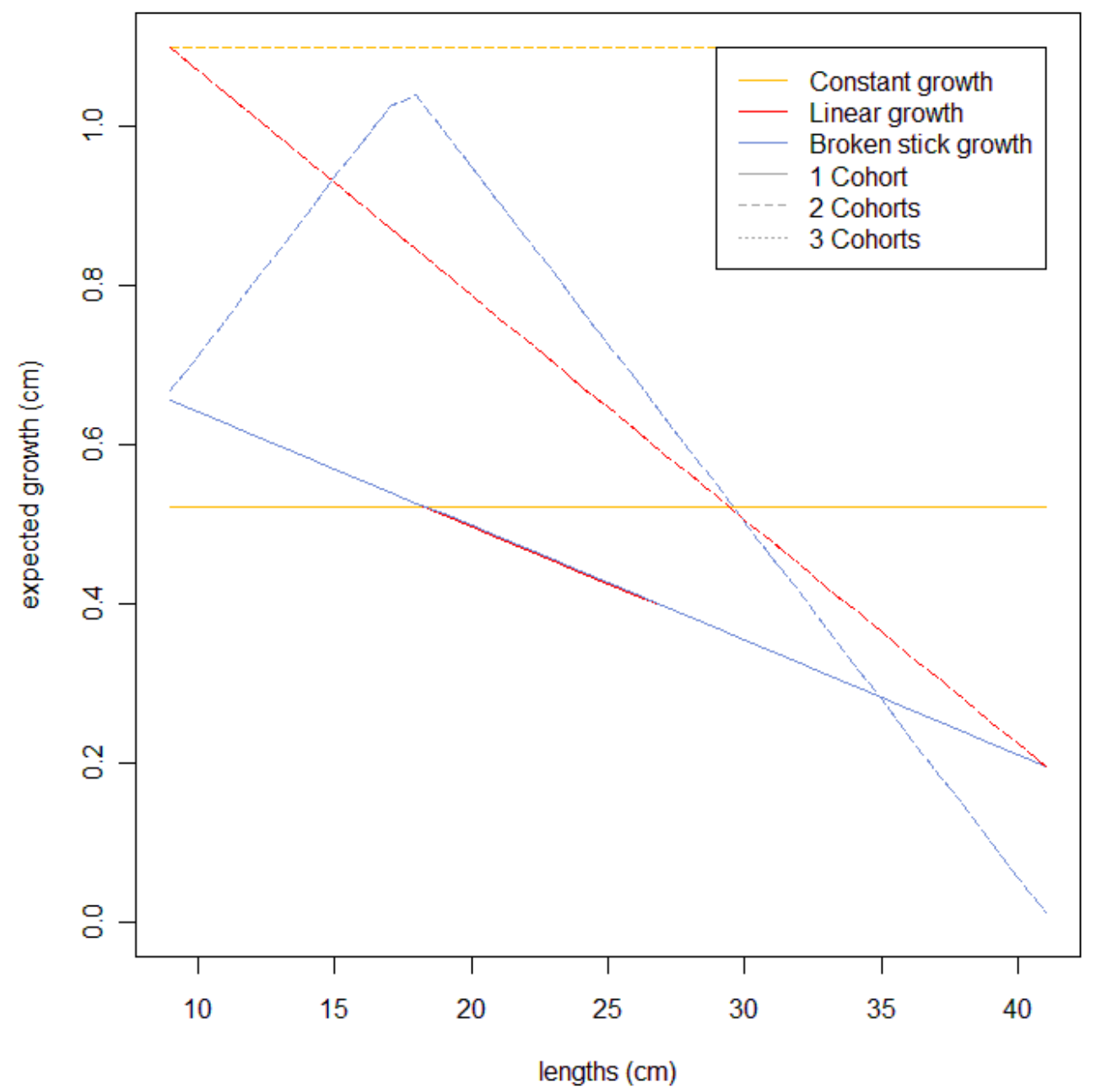

Figure B.4a: Expected mean growth by length for Snares 1990 from models with constant, linear and broken stick mean growth and 1, 2, and 3-cohorts. Mean growth is the mean growth for 1 week. 

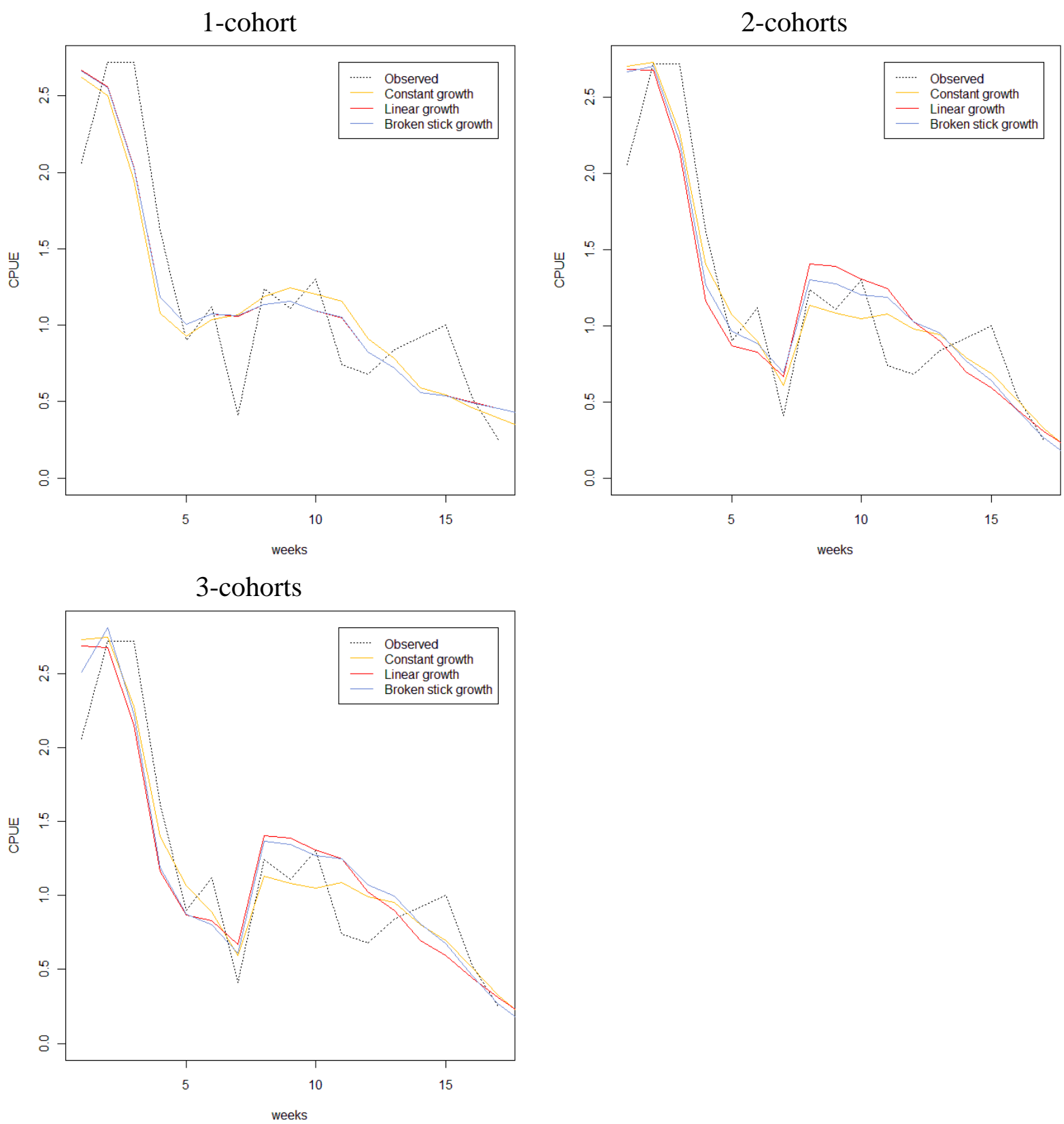

Figure 8.3.2a: Observed CPUE compared to expected $q \times$ exploitable biomass for Snares 1990, for constant, linear and broken stick mean growth and 1,2 and 3-cohorts. 
1-cohort

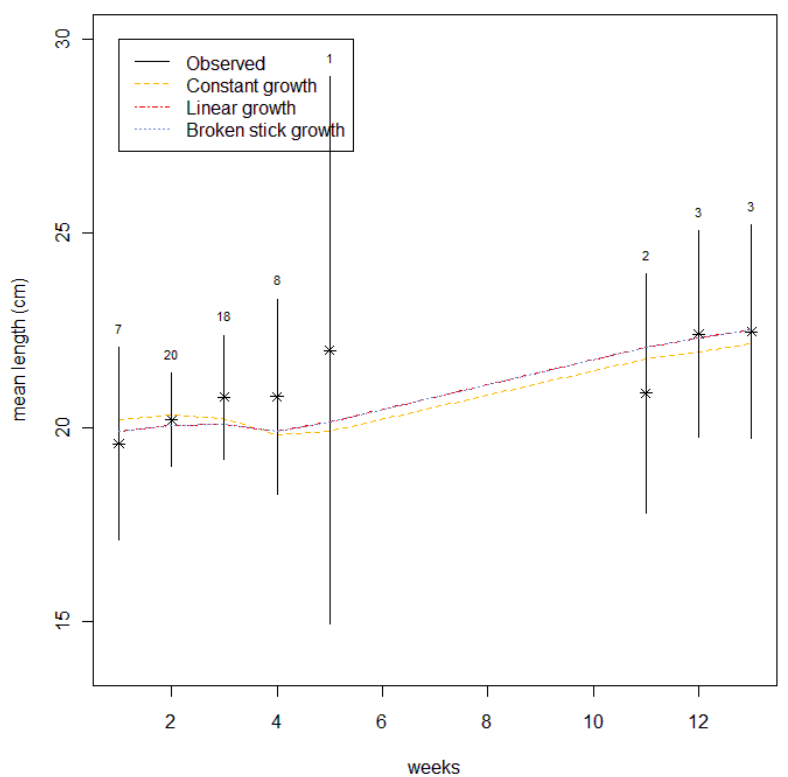

3-cohorts

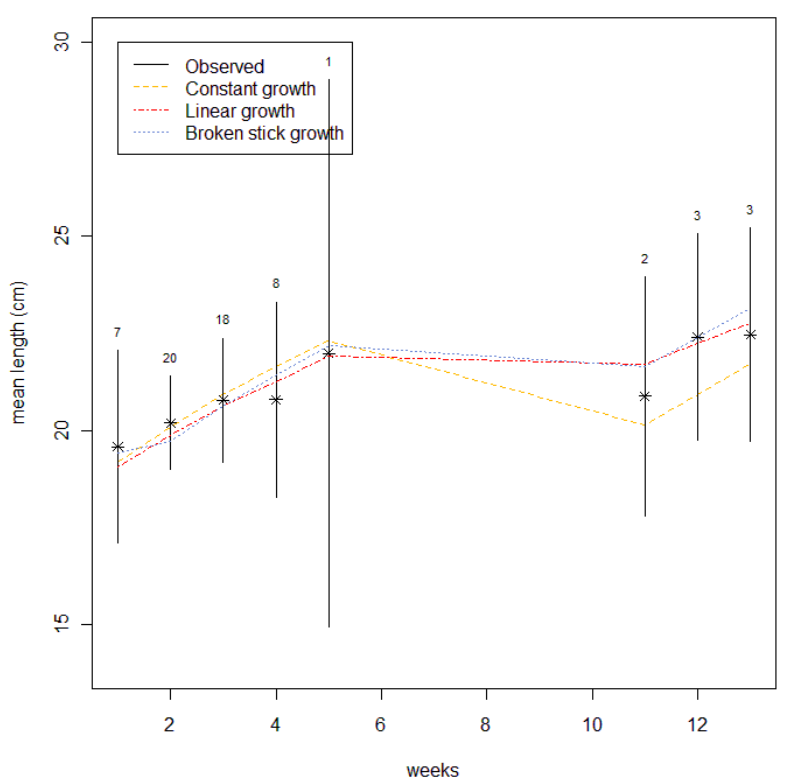

2-cohorts

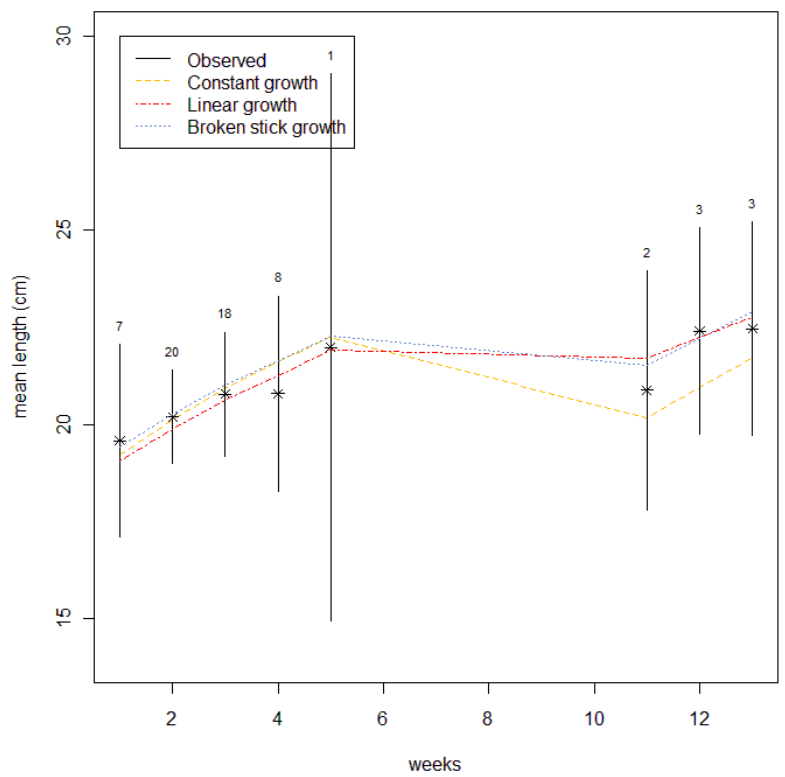

Figure 8.3.2b: Observed mean lengths with $95 \%$ confidence intervals, compared to expected mean lengths for Snares 1990, for constant, linear and broken stick mean growth and 1, 2 and 3-cohorts. The vertical black lines show the $95 \%$ confidence intervals for the observations, calculated using the number of tows (the numbers above the black lines) as the actual sample sizes. 
1-cohort
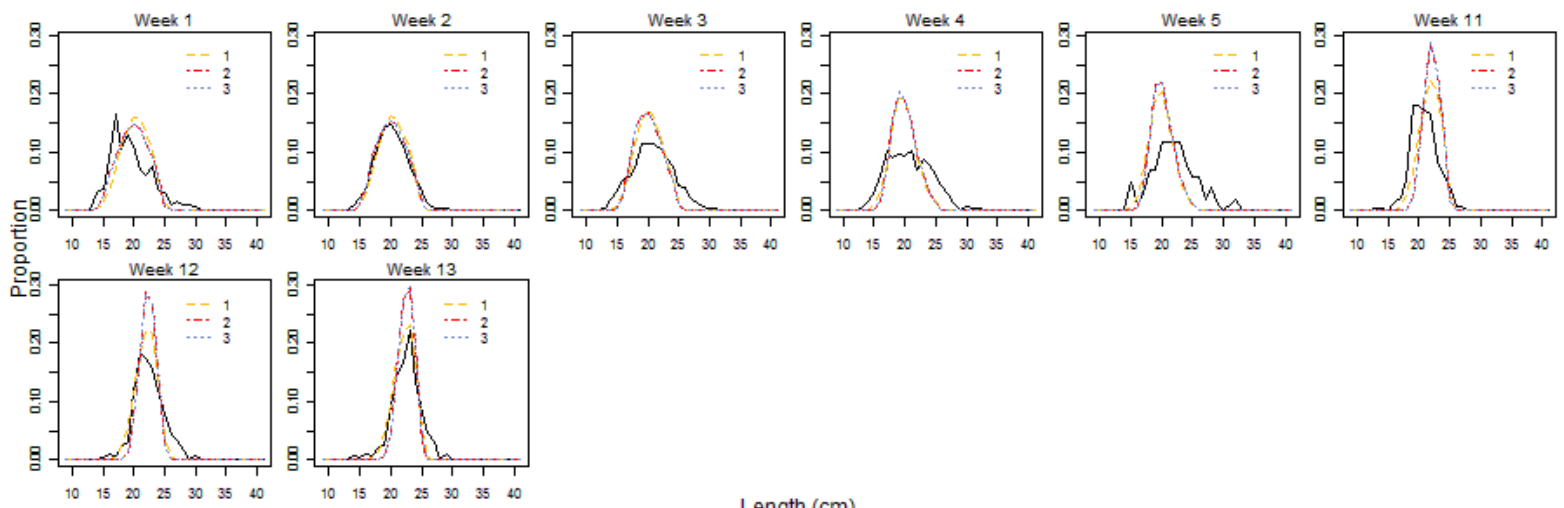

Week 1

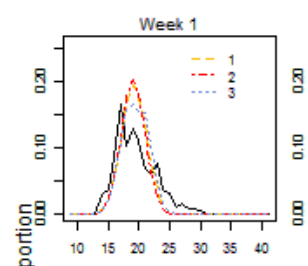

Week 2
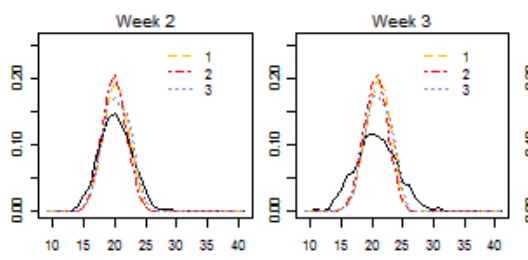

Length $(\mathrm{cm})$

2-cohorts
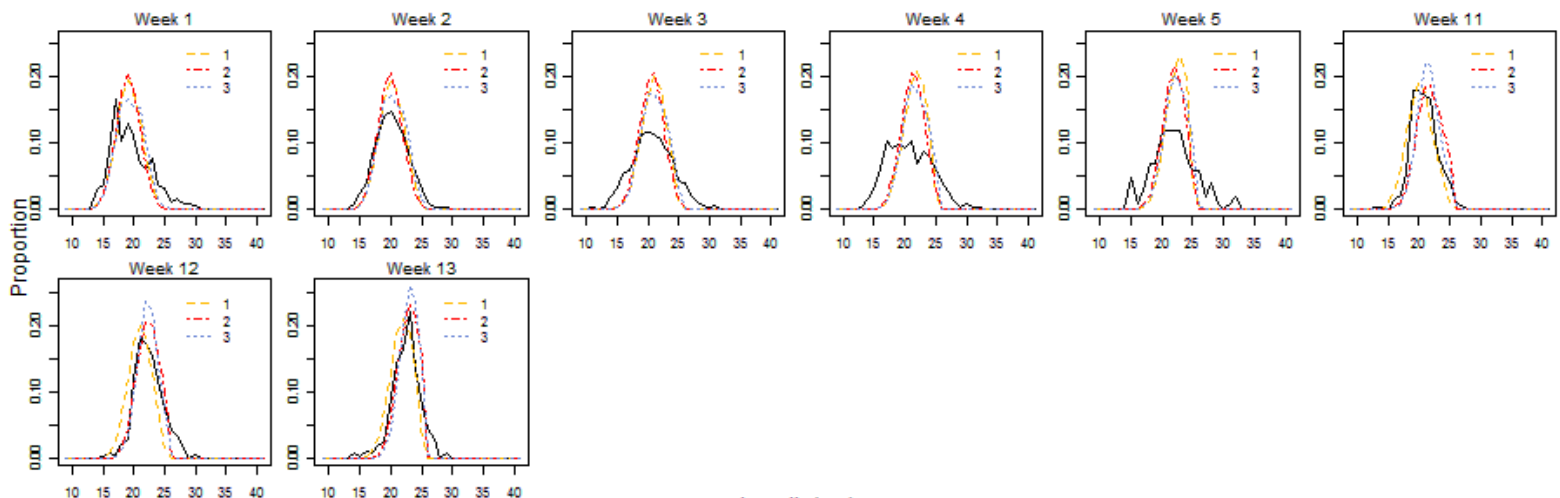

Length $(\mathrm{cm})$

3-cohorts
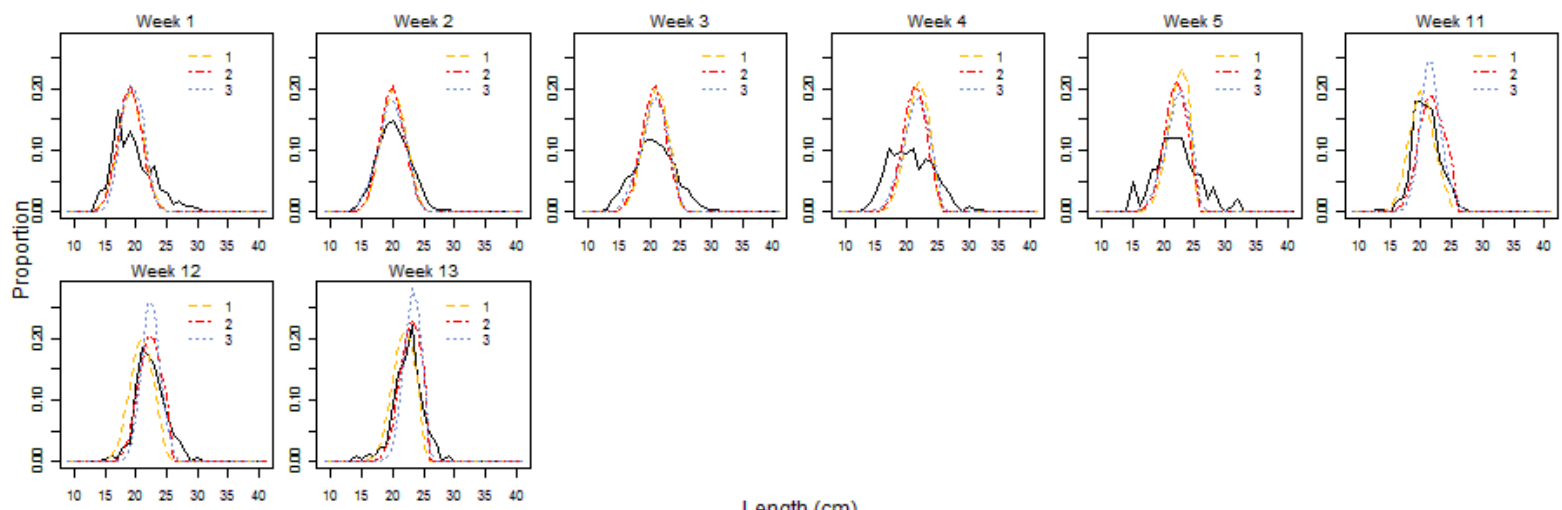

Length $(\mathrm{cm})$

Figure 8.3.: Observed length frequencies compared to expected length frequencies for Snares 1990, for 1, 2 , and 3 mean growth parameters and 1, 2 and 3-cohorts. Last plot shows the constant growth (2 mean growth parameters) model with 1, 2 and 3-cohorts. 
Appendix C

$\mathrm{R}$ code and ADMB code 
//finds optimised parameters for squid_model

DATA_SECTION

//data brought from .dat file

init_number nfw_data

init_vector data_fishing_weeks(1,nfw_data) //the fishing weeks we have proportions at

length data for. Often non continous and don't include the full season, as the catch

weight weeks do (below)

init_number nfw_cont

init_vector cont_fishing_weeks(1,nfw_cont) //continous version of the length frequency

data weeks

init_number nfw_all //number of fishing weeks for which we have catch weights

init_vector all_fishing_weeks(1,nfw_all) //these are all fishing weeks we have catch weights for (encompases both fishing weeks above)

init_number last_fw //the last fishing week

init_number nl //number of length bins

init_vector lengths $(1, \mathrm{nl})$ //the length bins

init_vector obs_weights(1,nfw_all) //observed catch weights for each week

init_number ncpw //number of cpue weeks

init_vector cpue_weeks $(1, \mathrm{ncpw})$ //weeks we have cpue indices for

init_vector cpue(1,ncpw) //the cpue indices

init_vector obs_catch_proportions(1,nl*nfw_data) //observed proportions at lenght (length frequency) data

init_number max_pop //size limit used for recruitment cohorts

init_vector N(1,nfw_data) //sample sizes (number of tows sampled from for each week)

init_vector 1 w_conversion $(1,2) / / a$ and $b$, the length to weight conversion parameters

init_number start_mu_sel //starting value for selectivity mean length

init_number nmg //number of mean growth parameters

init_number nc //number of cohorts

init_number rweek1_min //min and max values for recruitment weeks and sizes

init_number rweek1_max

init_number rweek2_min

init_number rweek2_max

init_number rweek3_min

init_number rweek3_max

init_number rweek1_start //start values for recruitment weeks and sizes

init_number rpop1_start

init_number rweek2_start

init_number rpop2_start

init_number rweek3_start

init_number rpop3_start

\section{PARAMETER_SECTION}

//THE PARAMETERS FOR ESTIMATING

// // estimate selectivity parameters

init_bounded_number mu_sel $(10,40,1)$

init_bounded_number sig_selL $(0.5,20,1)$

init_bounded_number sig_selR $(0.5,20,1)$

// // estimate growth parameters

init_bounded_number var_grow $(0.05,3,1)$

// 1 PARAMETER MEAN GROWTH - CONSTANT

init_bounded_number mg1 $(0.5,2.0,1)$

// // 2 PARAMTER MEAN GROWTH, - 1 STRAIGHT LINE 
init_bounded_number mg_start $(0.5,2,1)$

init_bounded_number mg_final $(0,1.5,1)$

// // //3 PARAMETER MEAN GROWTH, - 2 STRAIGHT LINES

init_bounded_number fnl $(0,1.5,1)$ / / flength_max). The growth rate at the last length for which we have LF data and length-weight conversion parameters. lengths [nl]

init_bounded_number $h(9,25,1) / / h$ in vertex parabola equation. The length at which the growth rate is at maximum or minimum

init_bounded_number $\mathrm{k}(0,2,1) / / \mathrm{k}$ in vertex parabola equation. The maximum or minimum growth rate

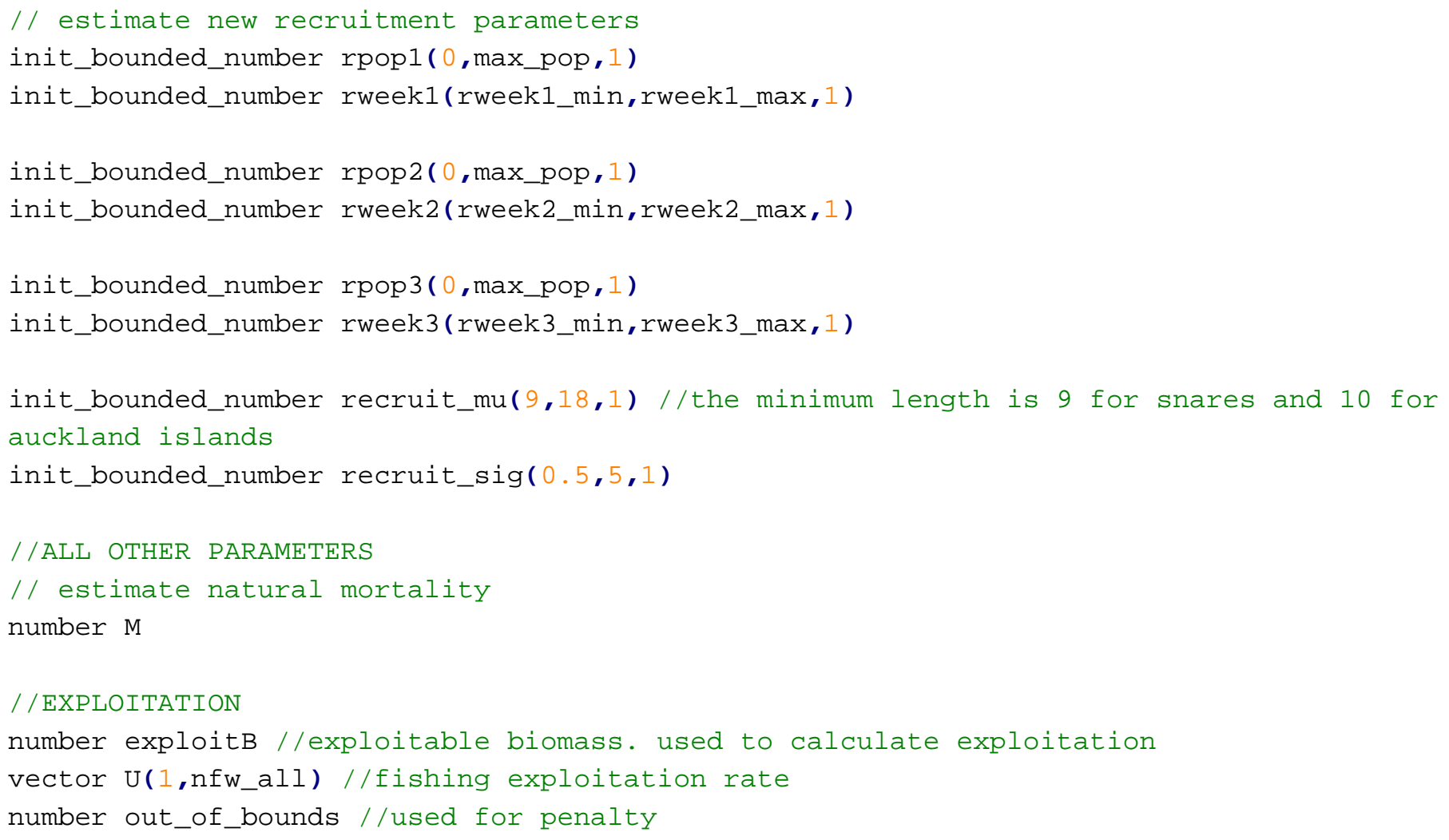

\section{/ / SELECTIVITY}

vector selectivity $(1, \mathrm{n} l)$

\section{/ /NEW RECRUITMENT}

number $\mathrm{nr}$

vector new_recruit $(1, \mathrm{nl})$ //for calculating new recruitment

vector new_recruit_grow $(1, \mathrm{nl})$ //used when growing the new recruit

vector recruit_growth_denom(1,nl) //recruitment growth denominator, used to normalise growth probability

number this_proportion //used to calculate the amount of growth for the new recruitment in it's first week (for when it comes in part way through a week)

number this_population //used when taking natural mortaltiy out of new recruitments vector this_weeks_total_recruitment $(1, \mathrm{nl})$ //needed for if there is more than 1 recruitment in a week

vector recruit_pops $(1,3)$

vector recruit_weeks $(1,3)$ 
vector temp_pop( $1, \mathrm{nl})$ //stores the current population for each week (it's replaced each week)

vector temp_pop_grow $(1, \mathrm{nl})$ //used to calculate population growth for each week (replaced each week)

/ /UNFISHED POPULATION and DEPLETION

vector temp_unfished_pop $(1, \mathrm{nl})$

vector total_unfished_population(1,nfw_all)

vector dep $\left(1, n f w \_a l l\right)$

\section{/ / GROWTH}

number this_grad //used in calculating linear mean growth 2 and 3 parameter number this_intercept //used in 2 parameter linear mean growth

number left_intercept //used in calculating mean growth

number right_intercept //used in calculating mean growth

number temp_sum //used when normalising new recruit that has just grown. stores the sum of the unnormalised grown vector

number this_sum //used to store the pre-growth sum of a population vector (temp_pop). Used for normalising the grown vector

number this_delta //used to store change in length

vector mean_growth_pars $(1,3)$ //stores mean growth parameters, just because it's easier for outputting them

vector ave_growth $(1, \mathrm{nl})$

number this_mean_growth

number this_a //used for parabolic mean growth

matrix temp_growth_mat $(1, \mathrm{nl}, 1, \mathrm{nl})$ //used to grow the squid

\section{/ /WEEKS}

number ffw //first fishing week

number last_week

number fw //fishing week

number first_week;

\section{//ESTIMATED CATCH WEIGHTS}

vector est_weights(1,nfw_all) //stores calculated estimated catch weights for each week

\section{//ESTIMATED CATCHES}

matrix pred_catch $(1, \mathrm{nl}, 1, \mathrm{nfw}$ _all) //stores the calculated catches (in NUMBERS) for each length and week

matrix E_matrix(1,nl,1,nfw_data) //stores estimated catches as proportions at length for each week that we have proportions at length data for

vector E(1,nl*nfw_data) // stores pred_catch as a vector of PROPORTIONS

number data_w //used to track the weeks for data_fishing_weeks (the weeks we have proportions at length data for)

\section{//STORED ESTIMATED POPULATION AND CATCH NUMBERS}

vector total_population(1,nfw_all) //weight

vector total_catch(1,nfw_all) //number

\section{//CPUE AND CATCHABILITY COEFFICIENT}

number q //catchability coefficient

vector expB(1,nfw_all) //exploitable biomass for each week

number $\mathrm{C} / / \mathrm{C} . \mathrm{V}$. of cpue, but will be estiamted here as 0.3

number cpw //counter for cpue weeks

number sum3 //sum 3 and 4 used to calculate $q$

number sum4

number sig2 //sigma squared, used in calculating $q$ 
number sig1 //square root of sig2

//calculatign difference of mean length for each week penalty

vector E_mean (1,nfw_data)

vector O_mean (1,nfw_data)

number mean_penalty

//CALCULATING OBJECTIVE FUNCTION

number this_weeks_sum1

number this_weeks_sum2

number this_z

number eps

number this_E_prime

number this_E

number this_o

number sum1

number sum2

objective_function_value f

\section{INITIALIZATION_SECTION}

//set the initial values for these parameters to those values created randomly in $\mathrm{R}$ and written to the dat file

rweek1 rweek1_start

rpop1 rpop1_start

rweek2 rweek2_start

rpop2 rpop2_start

rweek3 rweek3_start

rpop3 rpop3_start

mu_sel start_mu_sel

\section{PROCEDURE_SECTION}

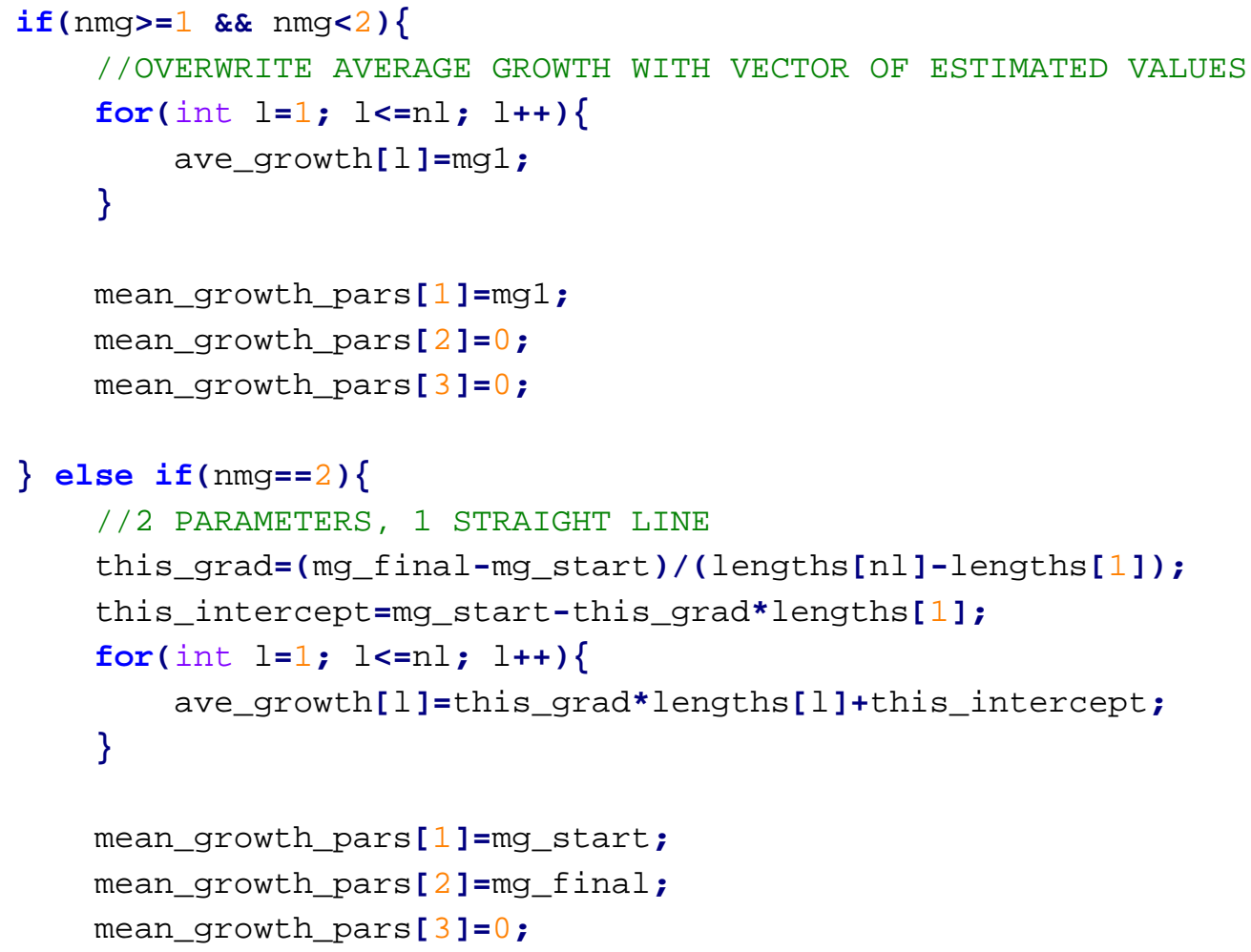


\} else if $(\mathrm{nmg}==3)$ \{

// // // 3 PARAMETERS TAKE 2, 2 STRAIGHT LINES

this_grad $=(\mathrm{k}-\mathrm{fnl}) /($ lengths [nl] $-\mathrm{h})$;

left_intercept $=\mathrm{k}-t h i s \_g r a d * h$;

right_intercept $=\mathrm{k}+$ this_grad*h;

for (int $l=1 ; l<=n l ; 1++)$ \{

if (lengths [1]<h) \{

ave_growth [1]=this_grad*lengths [1]+left_intercept;

\} else \{

ave_growth $[1]=-t h i s \_g r a d *$ lengths [1]+right_intercept ;

\}

\}

mean_growth_pars [1]=fnl;

mean_growth_pars [2] $=h$;

mean_growth_pars [3]=k;

\}

//population recruitment population and timing vectors

recruit_pops [1]=rpop1;

recruit_pops [2]=rpop2;

recruit_pops [3]=rpop3;

recruit_weeks [1] =rweek1;

recruit_weeks [2]=rweek2;

recruit_weeks [3]=rweek3;

//set natural mortality

$\mathrm{M}=0.017$;

//set length to weight conversion parameters

$\mathrm{a}=1 \mathrm{w}$ _conversion [1];

$\mathrm{b}=1 \mathrm{w} \_$conversion [2];

last_week=nfw_all;//max (data_fishing_weeks); CHANGE

first_week=-10;

//set objective function to 0 before we start

$\mathrm{f}=0$;

temp_pop=0;

temp_unfished_pop=0;

//calculate selectivity for each length and store in vector selectivity

for (int $l=1 ; l<=n l ; l++)\{$

if (lengths [1] $<=$ mu_sel) \{

selectivity [1] =pow $(2,-\operatorname{pow}((($ lengths [1]-mu_sel)/sig_selL), 2)) ;

\} else \{

selectivity [1]=pow (2,-pow ( ( lengths[1]-mu_sel)/sig_selR),2)); \}

\}

for (int $\mathrm{w}=(-10) ; \mathrm{w}<=$ value (last_week); $\mathrm{w}++)$ \{

this_weeks_total_recruitment $=0$; 
//if there is no population yet, skip to adding new recruitments

if ( sum (temp_pop) >0) \{

//if its not a fishing week, just take out natural mortality

if $(w<1)\{/ /$ we have weekly catch weight data from week 1

temp_pop=temp_pop*exp (-M) ;

// temp_pop=temp_pop;

\} else \{

//if it is a fishing week, take out catch and natural mortality

for (int $l=1 ; l<=n l ; 1++)$ l

total_population [w] +=temp_pop [1]*a*(pow (lengths [1],b)) ;

total_unfished_population [w]+=temp_unfished_pop[1]*a*(pow (lengths [1],b)) ;

\}

//ready to calculate total estimated catch weight for this week

est_weights $[\mathrm{w}]=0$;

//calculate exploitation rate

$\mathrm{U}[\mathrm{w}]=0 ; / /$ set exploitation rate to 0

exploit $\mathrm{B}=0 ; / /$ set exploitatble biomass to 0

for (int $1=1 ; 1<=n l ; 1++$ )

//calculate exploitable biomass

exploitB+=temp_pop [1]*exp (-M)*selectivity [1]*a* (pow(lengths [1],b));

\}

$\operatorname{expB}[\mathrm{w}]=\operatorname{exploitB}$

if (exploitB>0) \{

//calculate exploitation rate, providing exploitable biomass is greater than 0

$\mathrm{U}[\mathrm{w}]=$ obs_weights $[\mathrm{w}] / \operatorname{exploitB;}$

\}

//restrict the exploitation rate to being less than 0.9

if $(U[w]>0.9)\{$

$\mathrm{U}[\mathrm{w}]=0.9$;

//add to the objective function as a penalty for wanting to stray

outside the exploitation limit

$\mathrm{f}=\mathrm{f}+1000$;

\}

//calculate catch and new population

for (int $1=1 ; l<=n l ; 1++$ ) ,

//estimated catch in numbers for this week and length

pred_catch $[1][\mathrm{w}]=$ temp_pop $[1] * \exp (-\mathrm{M}) *$ selectivity $[1] * U[\mathrm{w}]$;

//estimated population in numbers for this week and length, with catch and natural mortality removed

temp_pop [1] =temp_pop [1]*exp (-M)-pred_catch [1] [w] ;

//estimated population in numbers for this week and length, with natural mortality removed, but NOT CATCH

temp_unfished_pop[1]=temp_unfished_pop [1] *exp (-M) ;

//store total estimated catch weight for this week

est_weights $[\mathrm{w}]=e s t \_w e i g h t s[w]+p r e d \_c a t c h[1][w] * a *(p o w(l e n g t h s[1], b))$; 
//store total catch in numbers for this week

total_catch $[\mathrm{w}]=\mathrm{col}$ sum $($ pred_catch,w) ;

\}

\}

//calculate any recruits that come in this week and grow accordingly

for (int $r=1 ; r<=n c ; r++)\}$

new_recruit $=0$;

if (w<=recruit_weeks[r] \&\& w+1>recruit_weeks[r]) \{

//how much the fish grow this week depends on how far through the week they arrive

this_proportion=w+1-recruit_weeks[r];

this_population=recruit_pops [r];

if (this_population>0) \{

//populate new recruitment with normal distribution

for (int $1=1 ; 1<=n l ; 1++)\{$

new_recruit [1] =1/(pow (2*M_PI, 0.5)*recruit_sig)*exp (-pow (

recruit_mu-lengths $[1], 2) /(2 *$ pow (recruit_sig, 2)))*this_population;

\}

//calculate the new post growth recruitment

new_recruit_grow $=0$ *new_recruit;

for (int $1=1 ; 1<=\mathrm{n} 1 ; 1++)\}$

for (int $k=1 ; k<=1 ; k++$ ) ;

this_mean_growth=ave_growth $[\mathrm{k}]$;

new_recruit_grow [1] +=(1/pow (2*M_PI*pow(this_proportion, 2$)$ *

var_grow, 0.5))*(exp (-pow ((lengths [1]-lengths [k]-this_proportion*

this_mean_growth),2)/(2*pow(this_proportion, 2)*var_grow))) *

new_recruit [k];

\}

\}

//normalise new_recruit_grow

new_recruit $=0$ *new_recruit_grow;

temp_sum=sum (new_recruit_grow);

for (int $1=1 ; 1<=n l ; 1++)\{$

new_recruit [1]=(new_recruit_grow[1]/temp_sum)*this_population; \}

\}

//this weeks total recruitment is set back to 0 at the start of each week, then added to for any new recruits that come in

this_weeks_total_recruitment=this_weeks_total_recruitment+new_recruit;

\}

\}

//if isn't any population, don't grow it

if ( $\operatorname{sum}($ temp_pop) $>0)$ \{

if $(w==17)$ \{

test_growth1=temp_pop;

\} 


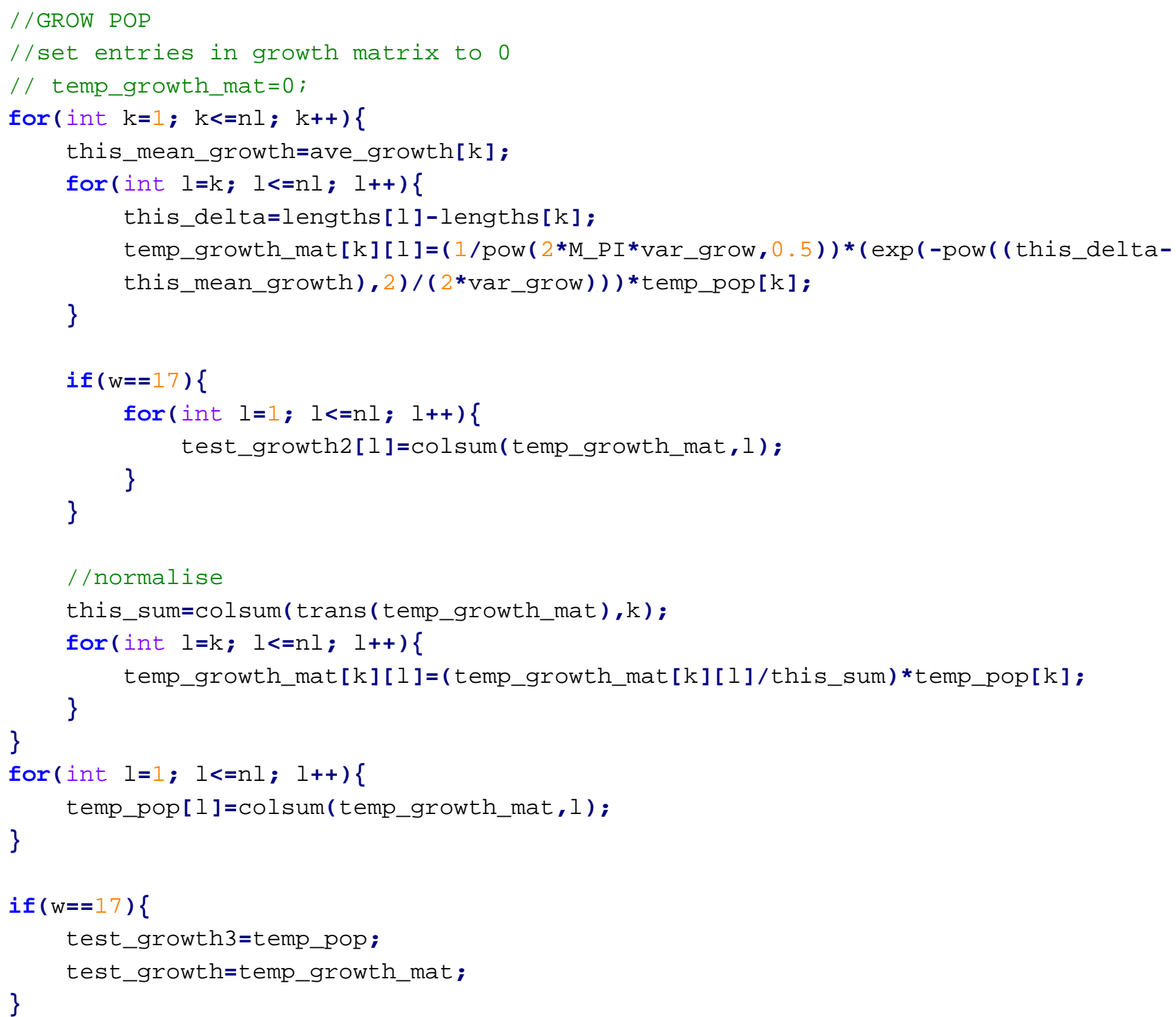


for (int $1=\mathrm{k} ; 1<=\mathrm{n} l ; 1++)\{$

temp_growth_mat [k][l]=(temp_growth_mat[k][l]/this_sum)*temp_unfished_pop[ $\mathrm{k}]$;

\}

\}

for (int $1=1 ; 1<=n l ; 1++)\{$

temp_unfished_pop [1] =colsum(temp_growth_mat, 1);

\}

\}

//ADD THE NEW RECRUITS TO THE NON-FISHED POPULATION TOO

//add the fish from the new recruitment to the existing population

for (int $1=1 ; 1<=n l ; 1++)\{$

temp_unfished_pop[1]=temp_unfished_pop[1]+this_weeks_total_recruitment[1];

\}

\}

//extract the predicted catches for weeks we have proportions data for

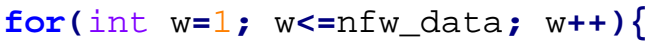

data_w=data_fishing_weeks[w]; //LF data fishing weeks often miss weeks. Using data_w only picks up the ones that correspond to the LF data fishing weeks

for (int $l=1 ; 1<=n l ; 1++$ ) \{

E_matrix [1] [w] =pred_catch [1] [value (data_w)];

\}

\}

$\mathrm{E}=0$;

//turn E_matrix into estimated catch proportions vector

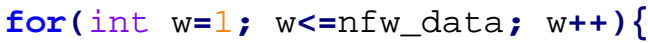

for (int $l=1 ; l<=n l ; l++)\{$

if (colsum (E_matrix,w) >0) \{

$\mathrm{E}\left[(\mathrm{w}-1) *_{\mathrm{n} l+1]}=\mathrm{E} \_\operatorname{matrix}[1][\mathrm{w}] / \mathrm{col} \operatorname{sum}\left(\mathrm{E} \_\operatorname{matrix}, \mathrm{w}\right)\right.$;

\}

\}

\}

//calculate FOURNIER likelihood

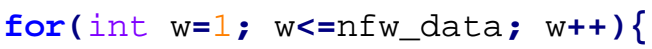

this_weeks_sum $1=0$;

this_weeks_sum2=0;

for (int $1=1 ; 1<=n 1 ; 1++)$ (

this_E $=E[(w-1) * n 1+1]$;

this_O=obs_catch_proportions $[(\mathrm{w}-1) * \mathrm{n} 1+1]$;

this_E_prime=(1-this_E)*this_E+0.1/nfw_data;

this_weeks_sum1+=log(this_E_prime);

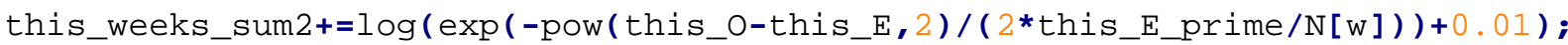

了

$\mathrm{f}=\mathrm{f}+0.5 *$ this_weeks_sum1-this_weeks_sum2;

\}

sig2 $=\log (1+0.09) ; / /$ this uses $c=0.3, c^{\wedge} 2=0.09$ 
\#read in real data, format it, write. dat file, then run admb optimiser and save results

\#select year and area to model

year<-"1990"

area<-"snar"

\#length weight conversion parameters

if $($ area $==$ ' snar') \}

squid_a $=0.0171$;

squid_b=3.08;

\} else \{

squid_a=0.0136

squid_b=3.16

\}

\#set paths

READ_PATH<-"C: \\squid2012\\LFData \\"

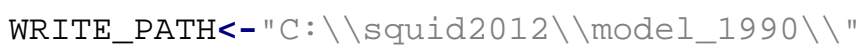

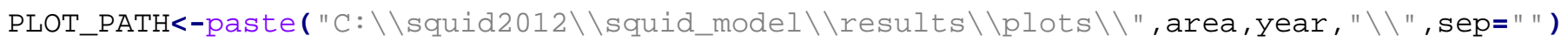

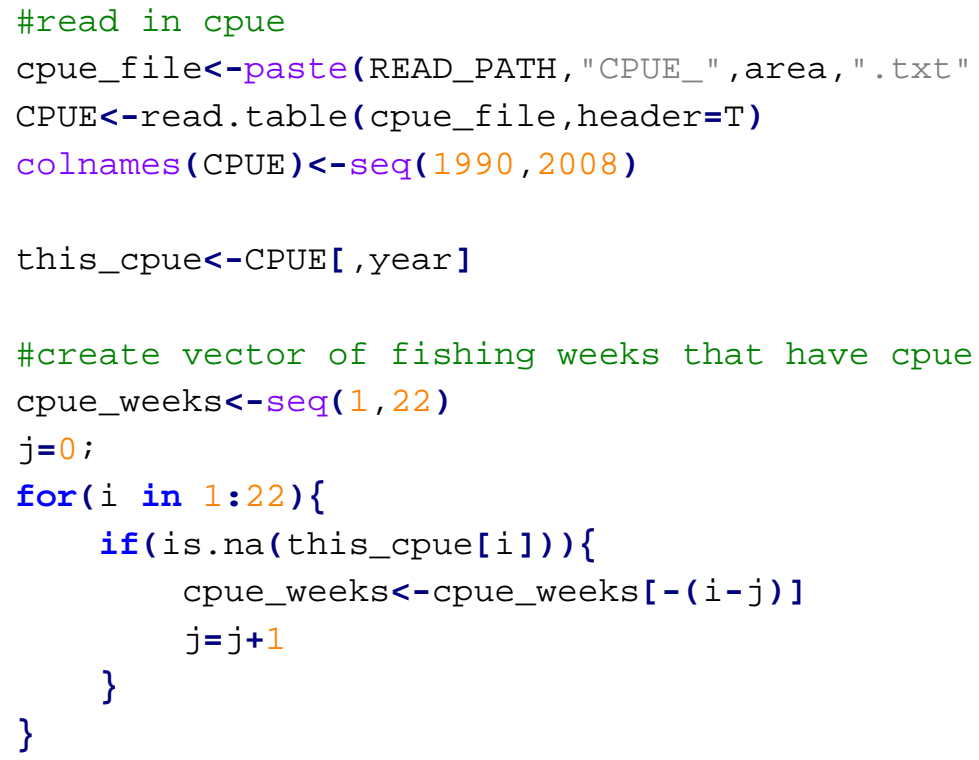




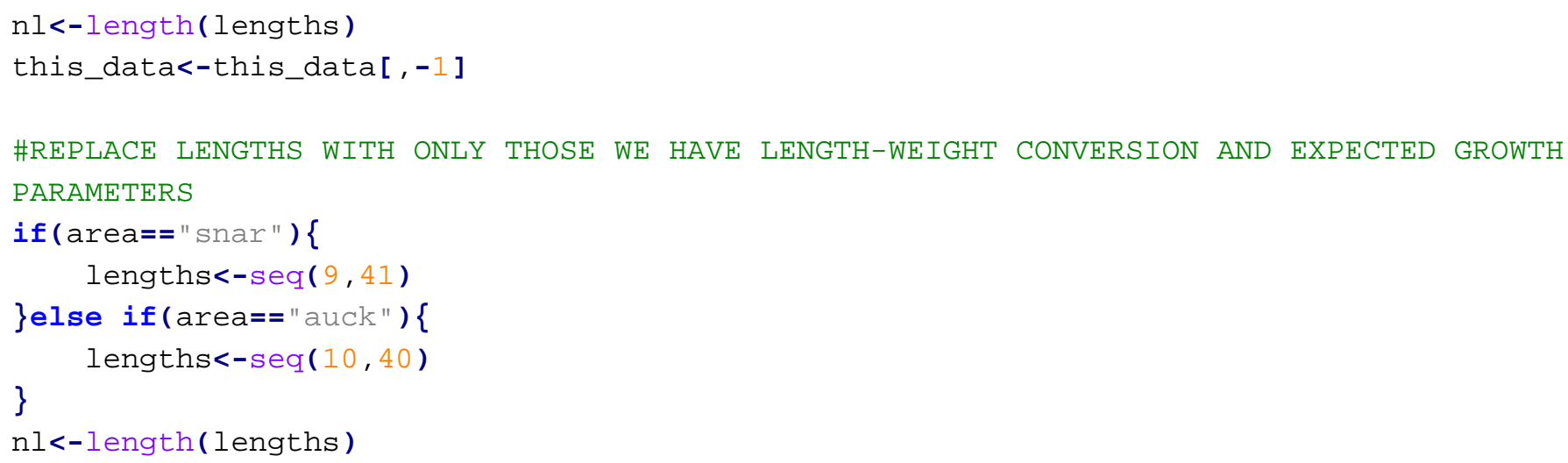




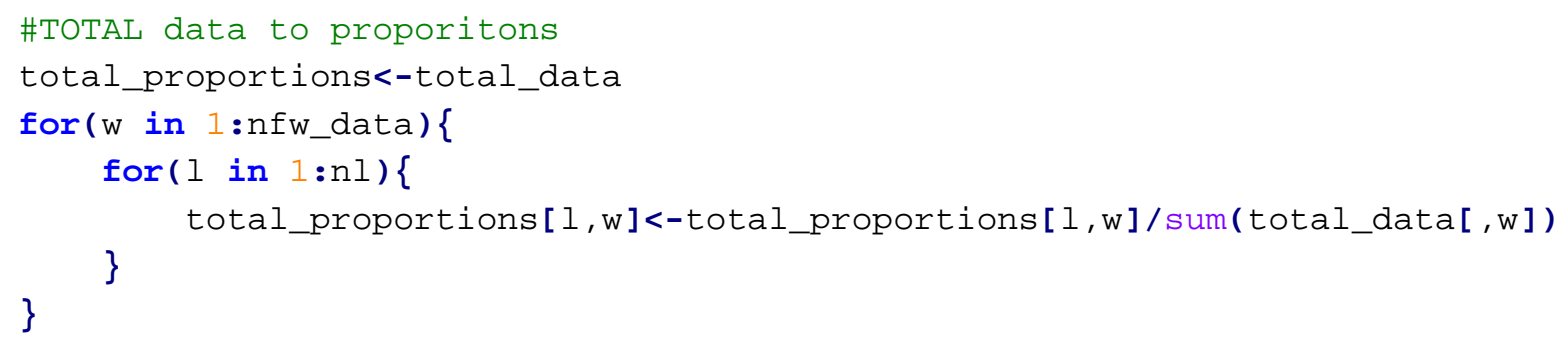


for ( $\mathrm{nmg}$ in $3: 3)$ f

for (nc in $3: 3)\{$

if (is.na (best_mat $[\mathrm{nmg}, \mathrm{nc}]))$ \{

this_output_file<-paste(PLOT_PATH,nmg," growth parameters \\", nc, " cohorts $\backslash \backslash$ ", sep=

") \#this will be used to output all files and plots relating to this run

\# set cohort week boundaries

rweek1_min<--10 \#the admb script starts at week -10

rweek1_max<-1 \#the first catches are in week 1, so need to have come population

by then

\#defalt for 2 nd and 3 rd cohort is to have them turned off and one way of doing

this is to have them come in at some week after the fishing season

rweek2_min<-rweek3_min<-24

rweek2_max<-rweek3_max<-25

if $(n c>1)$ i

rweek2_min $<-1$

rweek2_max<-max (data_fishing_weeks)

if ( $\mathrm{nc}>2$ ) \{

rweek3_min<-1

rweek3_max<-max(data_fishing_weeks)

\}

\}

\#set up variables to store current best objective function (f) and which loop it comes from

best $\mathrm{f}<-0$

bestp $<-0$

best_tn<-0

\#run the model $p$ times, using different starting values each time

for $(p$ in $1: 20)\{$

\# $\mathrm{p}=1$

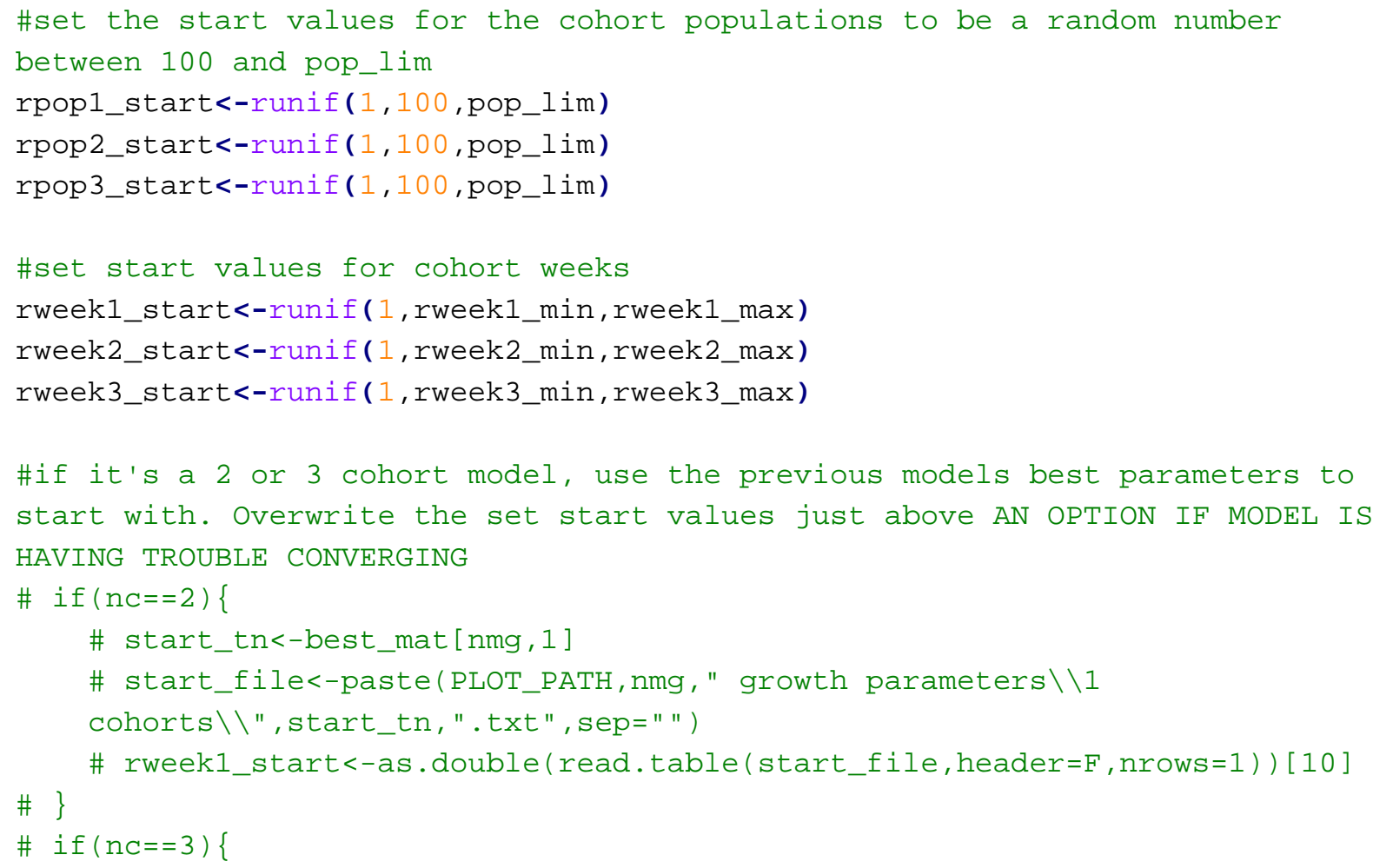




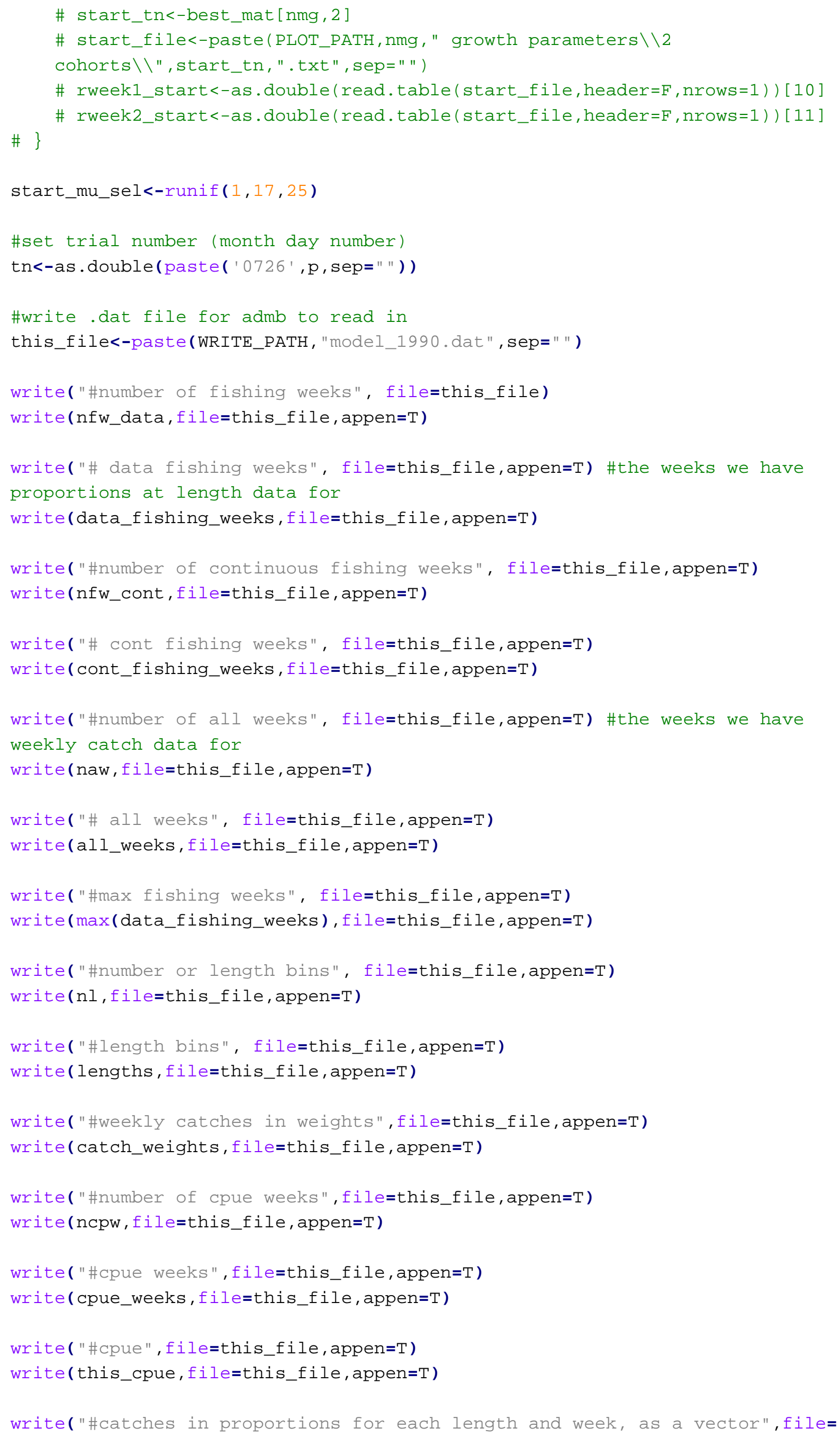


this_file, appen=T)

write(total_props_vec,file=this_file,ncolumns=nfw_data, appen=T)

write("\#population limit ", file=this_file, appen=T)

write (pop_lim,file=this_file, appen=T)

write ("\#N ", file=this_file, appen=T)

write (N,file=this_file, appen=T)

write("\#lw conversion parameters ", file=this_file, appen=T)

write(c(squid_a,squid_b),file=this_file, appen=T)

write("\#mu_sel ", file=this_file, appen=T)

write (start_mu_sel,file=this_file, appen=T)

write("\#number of mean growth pars ", file=this_file, appen=T)

write (nmg, file=this_file, appen=T)

write("\#number of cohorts ", file=this_file, appen=T)

write (nc,file=this_file, appen=T)

write("\#rweek1_min ", file=this_file, appen=T)

write (rweek1_min, file=this_file, appen=T)

write ("\#rweek1_max ", file=this_file, appen=T)

write (rweek1_max,file=this_file, appen=T)

write("\#rweek2_min ", file=this_file, appen=T)

write(rweek2_min, file=this_file, appen=T)

write("\#rweek2_max ", file=this_file, appen=T)

write(rweek2_max,file=this_file, appen=T)

write("\#rweek3_min ", file=this_file, appen=T)

write(rweek3_min,file=this_file, appen=T)

write ("\#rweek3_max ", file=this_file, appen=T)

write (rweek3_max, file=this_file, appen=T)

write ("\#rweek1_start ", file=this_file, appen=T)

write(rweek1_start, file=this_file, appen=T)

write("\#rpop1_start ", file=this_file, appen=T)

write(rpop1_start,file=this_file, appen=T)

write ("\#rweek2_start ", file=this_file, appen=T)

write(rweek2_start,file=this_file, appen=T)

write("\#rpop2_start ", file=this_file, appen=T)

write(rpop2_start, file=this_file, appen=T)

write ("\#rweek3_start ", file=this_file, appen=T)

write (rweek3_start,file=this_file, appen=T)

write("\#rpop3_start ", file=this_file, appen=T)

write(rpop3_start,file=this_file, appen=T) 


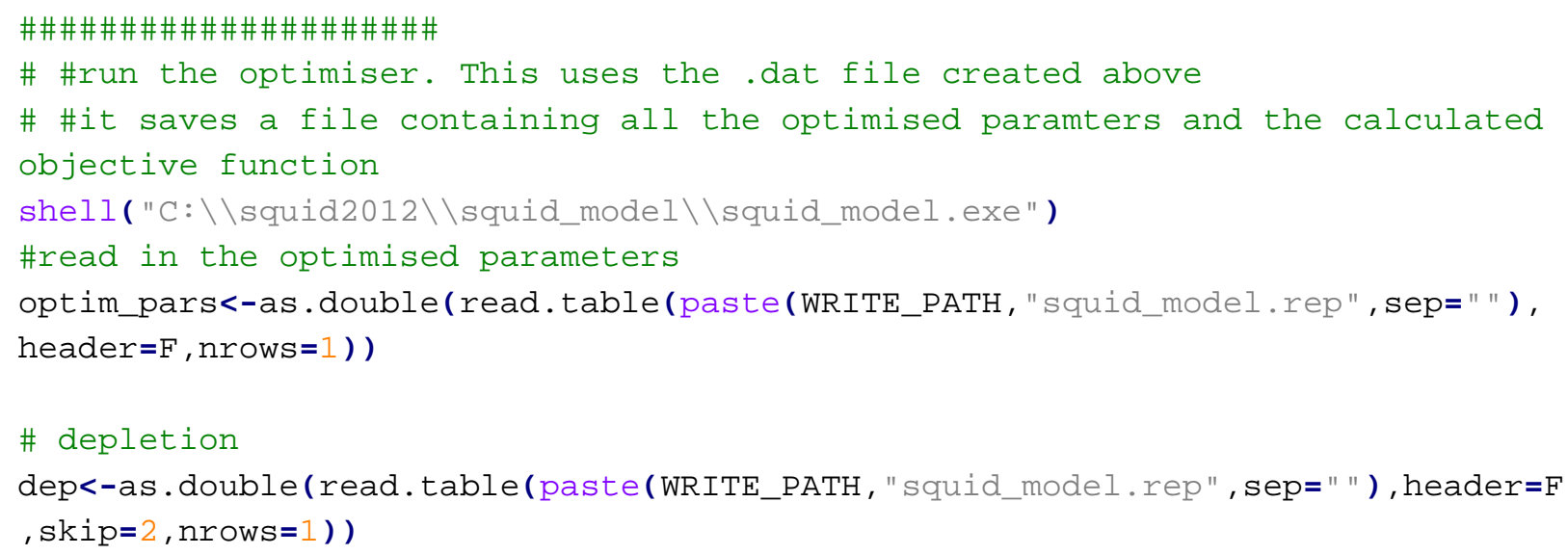




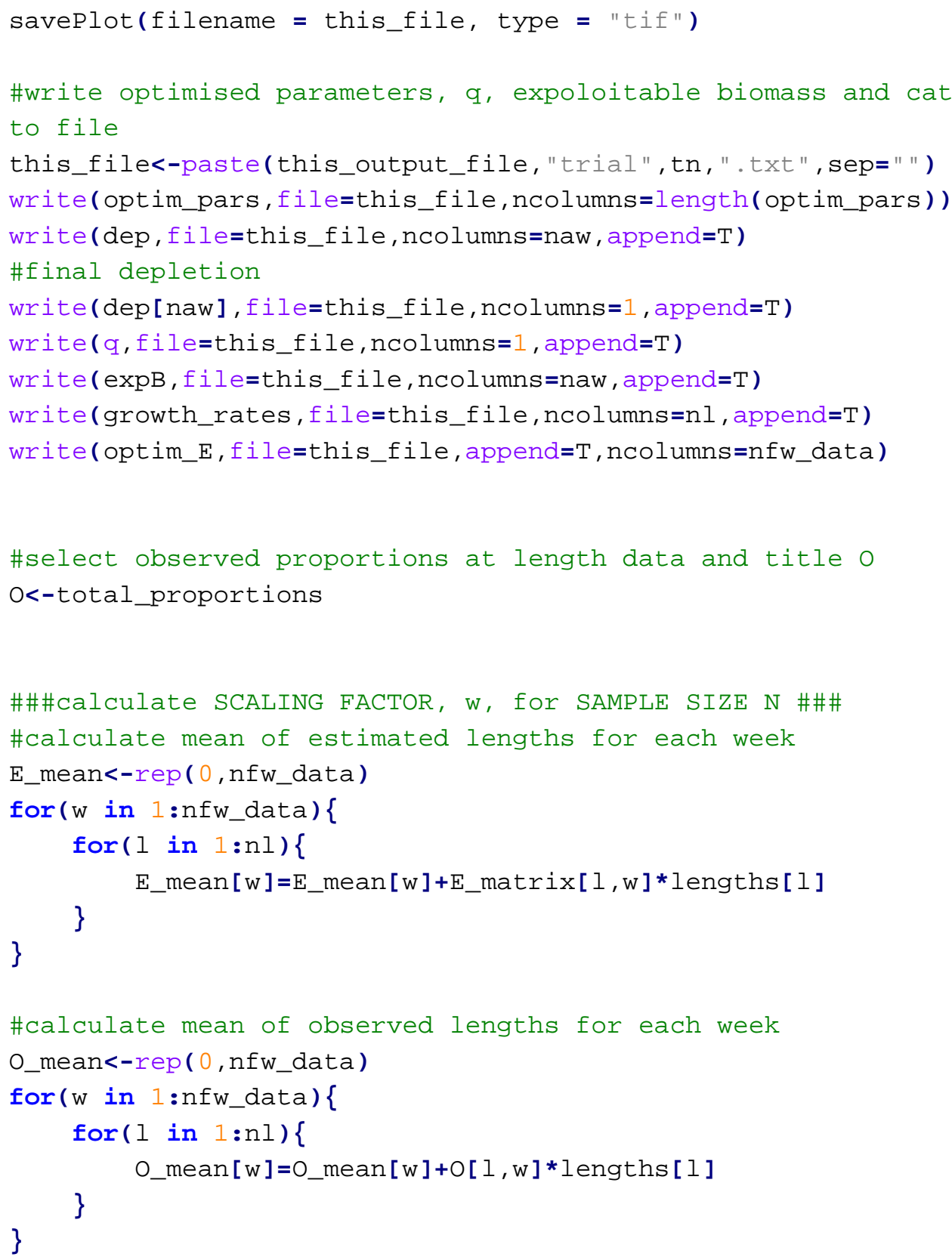




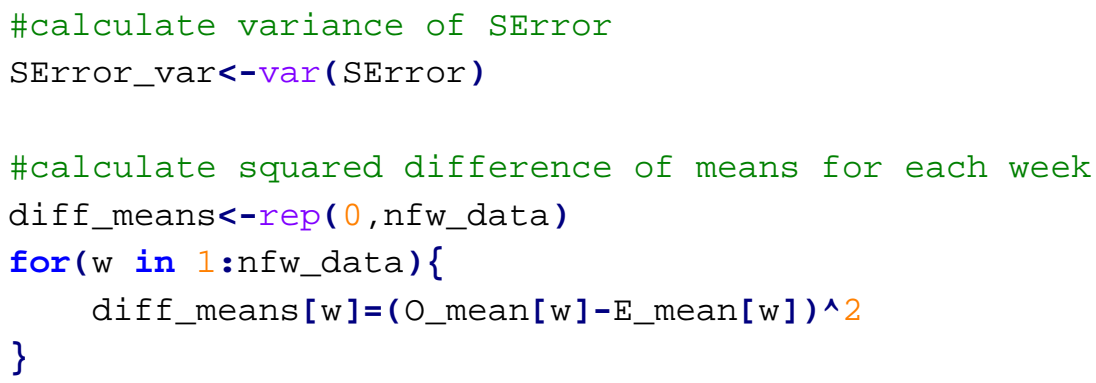


data_fishing_weeks[nfw_data], y=min (O_lower_CI) +0.5,pos=2)

text (paste ("f ", round (optim_pars [1],2), sep=" "), x=data_fishing_weeks[nfw_data] ,$y=\min ($ O_lower_CI), pos=2)

this_file<-paste(this_output_file,"trial",tn," Mean Lengths", sep=" ") savePlot (filename = this_file, type = "tif")

\}

best_mat [nmg, nc]<-paste ("trial", best_tn, sep=" ")

\} 
\#read in real data, format it, write. dat file, then run admb optimiser and save results \#this is very similar to run_squid_model.r, which was used for the snares data that wasn't combined with auckland islands

\# this one was only used for SNARES AND AUCKLAND ISLANDS 2008 COMBINED

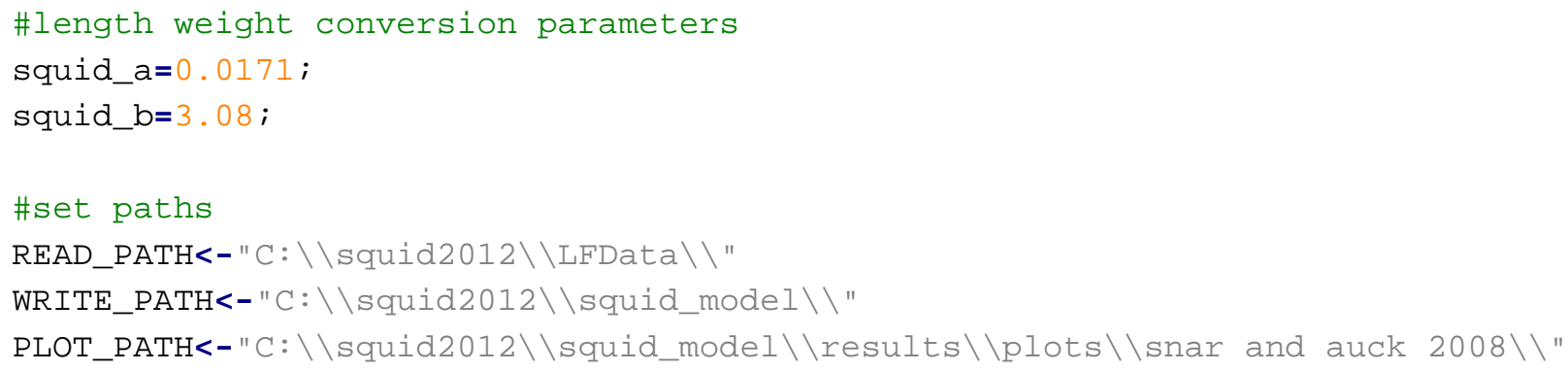


temp_this_cpue<-auck_cpue

auck_cpue<-0*auck_cpue_weeks

for ( $i$ in 1 :auck_ncpw) \{

auck_cpue[i]<-temp_this_cpue[auck_cpue_weeks[i]]

\}

\#SET LENGTHS AS ONLY THOSE WE HAVE LENGTH-WEIGHT CONVERSION AND EXPECTED GROWTH PARAMETERS

snar_lengths<-seg $(9,41)$

auck_lengths<-seg $(10,40)$

lengths<-seq $(10,40) \quad$ \# (same result)

seq (max (min (snar_lengths), min (auck_lengths)), min (max (snar_lengths), max (auck_lengths)) )

nl<-length (lengths)

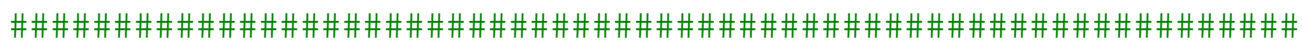

\#SNARES LENGTH FREQUENCY

\#read in the numbers at length for each week data

read_file<-paste ("SQUsnar2008byweek.txt", sep=" ")

snar_data<-read.table (paste (READ_PATH, read_file, sep=" "), header=T)

\#strip out lengths vector from the data and store

snar_data<-snar_data $[,-1]$

\#calculate the number of fishing weeks in the data (not necessarily continuous)

snar_nfw_data<-ncol (snar_data)/4-1

\#seperate total (males females combined) data for snares

sdata<-matrix $(0, \operatorname{ncol}=$ snar_nfw_data, nrow=nl)

for ( $w$ in 1:snar_nfw_data) \{

sdata $[, w]<-\operatorname{snar} d a t a[2: 32,(w * 4)]$

\}

\#seperate out fishing weeks

\#first, store the column headings which are 'total_1, total_2,...'

total_weeks<-rep (0,snar_nfw_data)

for (w in 1:snar_nfw_data) \{

total_weeks [w] <-colnames (snar_data) [w*4]

\}

\#now strip just the week numbers

sdata_fishing_weeks<-rep (0, snar_nfw_data)

max_nchar<-max (nchar (total_weeks))

min_nchar<-min (nchar(total_weeks))

for ( $w$ in 1 :snar_nfw_data) \{

sdata_fishing_weeks[w]<-as.double(substr(total_weeks[w], start=min_nchar, stop=max_nchar))

\}

\#create continuous fishing weeks vector

scont_fishing_weeks<-seq(min (sdata_fishing_weeks), max (sdata_fishing_weeks))

nfw_cont<-length (scont_fishing_weeks)

cw_file<-paste ("weekly_catches_snar_2008.txt", sep=" ")

scatch_weights<-as.double (read.table (paste (READ_PATH, Cw_file, sep=" "), header=F))

snaw<-length (scatch_weights)

sall_weeks<-seq $(1, \operatorname{snaw})$

\#add column headings to sdata

colnames (sdata) <-sdata_fishing_weeks 
\#calculate catch in numbers for each week. catch weights are in tonnes. catch numbers are in millions

scatch_numbers <-rep (0, snar_nfw_data)

dwc<-1 \#data week count

for ( $w$ in $1: \max ($ sdata_fishing_weeks))

if (sall_weeks $[\mathrm{w}]==$ sdata_fishing_weeks [dwc] ) \{

for $(1$ in $1: n l)$ (

this_weight<-scatch_weights[w]*sdata [1,dwc]/sum (sdata [, dwc])

scatch_numbers [dwc]<-scatch_numbers[dwc]+this_weight/(a*lengths[1]^b) \}

\#increase data weeks counter

$d w c<-d w c+1$

\}

\}

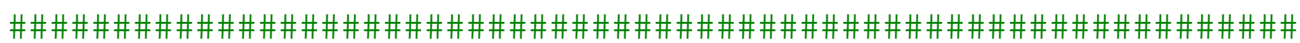

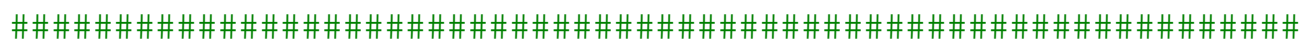

\#AUCKLAND ISLANDS LENGTH FREQUENCY

\#read in the numbers at length for each week data

read_file<-paste ("SQUauck2008byweek.txt", sep=" ")

auck_data<-read.table (paste (READ_PATH, read_file, sep=" "), header=T)

\#strip out lengths vector from the data and store

auck_data<-auck_data $[,-1]$

\#calculate the number of fishing weeks in the data (not necessarily continuous)

auck_nfw_data<-ncol(auck_data)/4-1

\#seperate total (males females combined) data for snares

adata<-matrix $\left(0, \operatorname{ncol}=a u c k \_n f w \_d a t a, n r o w=n l\right)$

for ( $w$ in 1 :auck_nfw_data) \{

adata $[, w]<-a u c k \_d a t a[m i n($ lengths) : $\max ($ lengths), (w*4)]

\}

\#seperate out fishing weeks

\#first, store the column headings which are 'total_1, total_2,...'

atotal_weeks<-rep (0, auck_nfw_data)

for ( $w$ in 1:auck_nfw_data) \{

atotal_weeks [w]<-colnames (auck_data) [w*4]

\}

\#now strip just the week numbers

adata_fishing_weeks<-rep (0,auck_nfw_data)

max_nchar<-max (nchar (atotal_weeks))

min_nchar<-min (nchar (atotal_weeks))

for ( $w$ in 1:auck_nfw_data) \{

adata_fishing_weeks[w]<-as.double(substr(atotal_weeks[w], start=min_nchar, stop=max_nchar))

\}

\#create continuous fishing weeks vector

acont_fishing_weeks<-seq(min (adata_fishing_weeks), max (adata_fishing_weeks))

anfw_cont<-length (acont_fishing_weeks)

CW_file<-paste ("weekly_catches_auck_2008.txt",sep=" ")

acatch_weights<-as.double (read.table (paste (READ_PATH, cw_file,sep=" "), header=F))

anaw<-length (acatch_weights)

aal1_weeks<-seq(1, anaw)

\#add column headings to sdata 
\#calculate catch in numbers for each week. catch weights are in tonnes. catch numbers are in millions

acatch_numbers $<-r e p(0$, auck_nfw_data)

dwc<-1 \#data week count

for ( $w$ in $1: \max ($ adata_fishing_weeks)) \{

if (aall_weeks[w] ==adata_fishing_weeks[dwc]) \{

for $(1$ in $1: n l)\{$

this_weight<-acatch_weights[w]*adata [1,dwc]/sum (adata [,dwc])

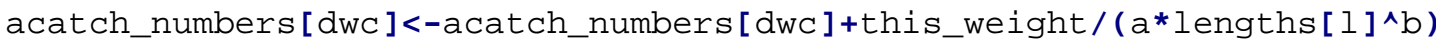

了

\#increase data weeks counter

$d w c<-d w c+1$

\}

\}

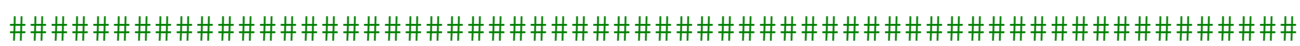

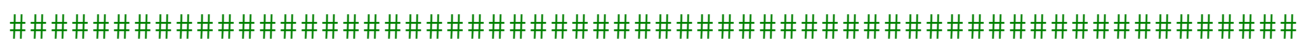

\# > sdata_fishing_weeks

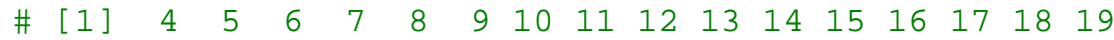

\# > adata_fishing_weeks

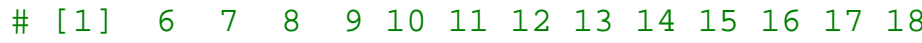

\# > sall_weeks

$\begin{array}{llllllllllllllllllllllll}\text { \# } & {[1]} & 1 & 2 & 3 & 4 & 5 & 6 & 7 & 8 & 9 & 10 & 11 & 12 & 13 & 14 & 15 & 16 & 17 & 18 & 19 & 20 & 21 & 22\end{array}$

\# > aall_weeks

$\begin{array}{lllllllllllllllllllllll}\text { \# }[1] & 1 & 2 & 3 & 4 & 5 & 6 & 7 & 8 & 9 & 10 & 11 & 12 & 13 & 14 & 15 & 16 & 17 & 18 & 19 & 20 & 21 & 22\end{array}$

\# > scont_fishing_weeks

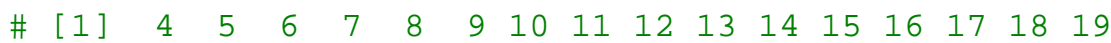

\# > acont_fishing_weeks

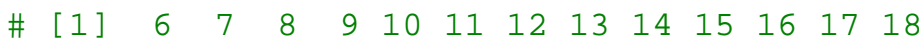

data_fishing_weeks<-seq $(4,19)$

nfw_data<-length (data_fishing_weeks)

all_weeks<-seq $(1,22)$

naw<-length (all_weeks)

cont_fishing_weeks<-seq $(4,19)$

nfw_cont<-length (cont_fishing_weeks)

\#combine length frequencies from snares and auckland islands

comb_data<-sdata

\#also combine catch numbers at the same time

catch_numbers<-scatch_numbers

$a<-1$

for ( $w$ in $\left.1: n f w \_d a t a\right)$ \{

aw<-adata_fishing_weeks [a]

sw<-sdata_fishing_weeks [w]

if $(a w==s w)$ \{

comb_data $[, w]<-c o m b \_d a t a[, w]+\operatorname{adata}[, \mathrm{a}]$

catch_numbers [w] <-catch_numbers [w] +acatch_numbers [a]

print (c ( $w, "$ ", sw, " ", aw))

$a=a+1$ 
\}

\#turn the combined data into proportions and then a vector proportions<-comb_data

for ( $w$ in $\left.1: n f w \_d a t a\right) ~\{$

for ( 1 in $1: n l)\{$

proportions [1,w]<-proportions [1,w] /sum (comb_data [,w])

\}

\}

\# and into a vector

props_vec<-rep $(0, \mathrm{nfw}$ _data*nl)

for ( $w$ in $\left.1: n f w \_d a t a\right) ~\{$

for ( 1 in $1: n l)$ \{

props_vec $[(w-1) * n l+1]<-p r o p o r t i o n s[1, w]$

\}

\}

\#write observed proportions to file

this_file<-paste (WRITE_PATH, "observed_proportions.txt", sep=" ")

write (props_vec,file=this_file,ncolumns=nl)

\#combine weekly catch weights

catch_weights<-scatch_weights+acatch_weights

\#combine CPUE

\# > auck_cpue_weeks

$\begin{array}{llllllllllll}\text { \# } & {[1]} & 8 & 9 & 10 & 11 & 12 & 13 & 14 & 15 & 16 & 17\end{array}$

\# > snar_cpue_weeks

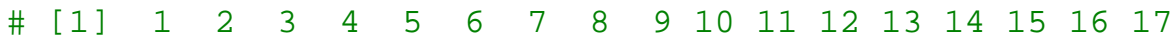

cpue_weeks<-seq $(1,17)$

ncpw<-length (cpue_weeks)

cpue<-snar_cpue

$a<-1$

for $(w$ in $1: n c p w)\{$

cw<-cpue_weeks [w]

aw<-auck_cpue_weeks[a]

this_sum<-catch_weights $[w]$

if $(\mathrm{CW}==\mathrm{aw}) \mathfrak{f}$

print (c (auck_cpue [a]," ",a))

cpue [w]<-(scatch_weights[w]/this_sum)*cpue[w]+(acatch_weights[w]/this_sum)*auck_cpue[ a]

$a<-a+1$

\}

\}

\#write observed cpue to file

this_file<-paste(WRITE_PATH, "cpue.txt", sep=" ") 
write (cpue, file=this_file, ncolumns=ncpw)

\#print new cpue and save ONLY INCLUDED THIS THE FIRST TIME IT RAN

\# ymax<-max (cpue, snar_cpue, auck_cpue)

\#

plot (snar_cpue_weeks, snar_cpue, type='l' , col=' red',ylim=c (0,ymax), lty=3, xlab='weeks',ylab='CPuE ')

\# points (auck_cpue_weeks, auck_cpue,type='l',col='cornflowerblue', lty=3)

\# points (cpue_weeks, cpue, type='l', ylim=c $(0, y m a x), 1 t y=2)$

\# legend(legend=C ("Snares CPUE", "Auckland Islands CPUE", "Combined

CPUE"), $1 t_{\mathrm{y}=\mathrm{C}}(3,3,2), \mathrm{col}=\mathrm{c}($ 'red' ' ' cornflowerblue' ' 'black'), $\mathrm{x}=1, \mathrm{y}=0.5, \mathrm{xjust=0})$

\# this_file<-paste (PLOT_PATH, "CPUEplot", sep=" ")

\# savePlot (filename = this_file, type = "tif")

\#set up variables to store $f$ and var(standardised errors of mean lengths)

best $<-0$

bestp $<-0$

$\mathrm{N}<-\mathrm{C}(10,37,55,57,42,42,46,33,45,12,15,7,6,13,7,1)$

\#calculate pop_lim as 20*total number caught

pop_lim<-1000

\#set up matrix to store filename of best parameters for each nc/nmg model

best_mat<-matrix (NA, ncol=3,nrow $=3)$

for (nmg in $3: 3)$ f

for (nc in $2: 2$ ) 1

if (is.na (best_mat [nmg,nc])) \{

this_output_file<-paste(PLOT_PATH,nmg," growth parameters \\", nc, " cohorts $\backslash \backslash$ ", sep= ") \#this will be used to output all files and plots relating to this run

\# set cohort week boundaries

rweek1_min<--10 \#the admb script starts at week -10

rweek1_max<-1 \#the first catches are in week 1, so need to have come population

by then

\#defalt for 2nd and 3rd cohort is to have them turned off and one way of doing

this is to have them come in at some week after the fishing season

rweek2_min<-rweek3_min<-24

rweek2_max<-rweek3_max<-25

if $(n c>1)$ f

rweek2_min<-1

rweek2_max<-max (data_fishing_weeks)

if $(n c>2)$ \{

rweek3_min<-1

rweek3_max<-max (data_fishing_weeks)

\} 
\# set up variables to store current best objective function (f) and which loop it comes from

bestf $<-0$

bestp $<-0$

best_tn<-0

\#run the model p times, using different starting values each time

for $(p$ in $1: 30)\{$

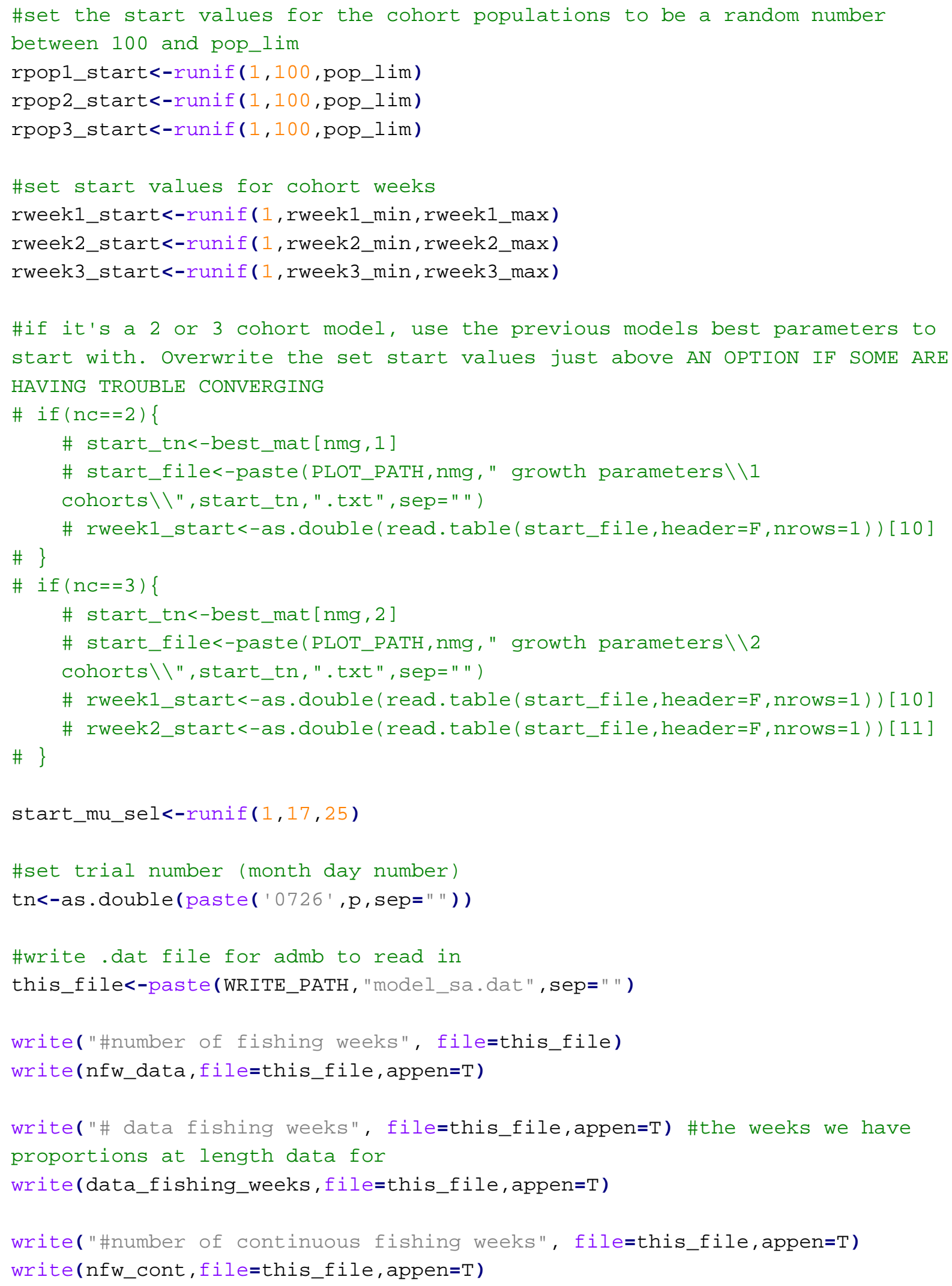




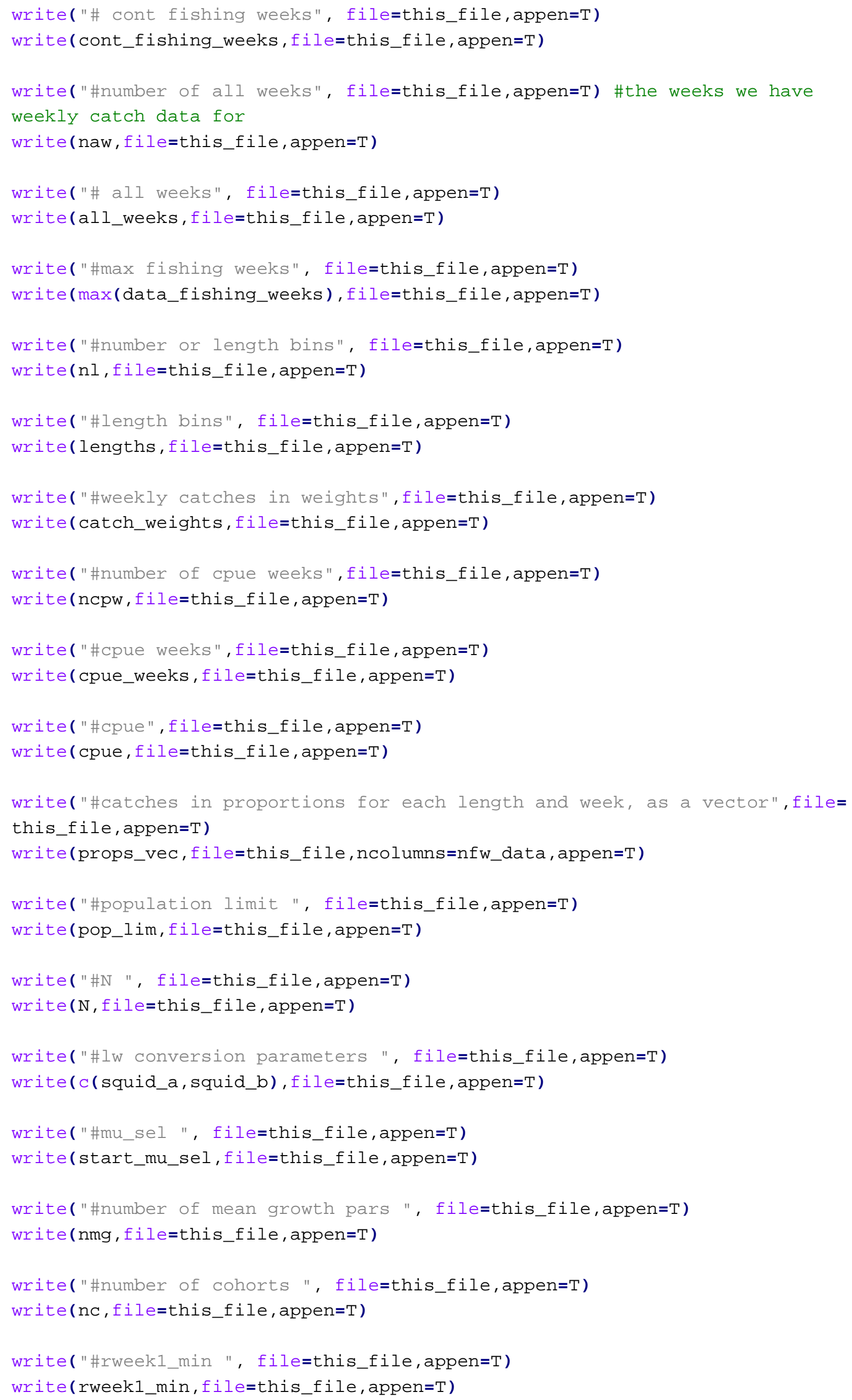




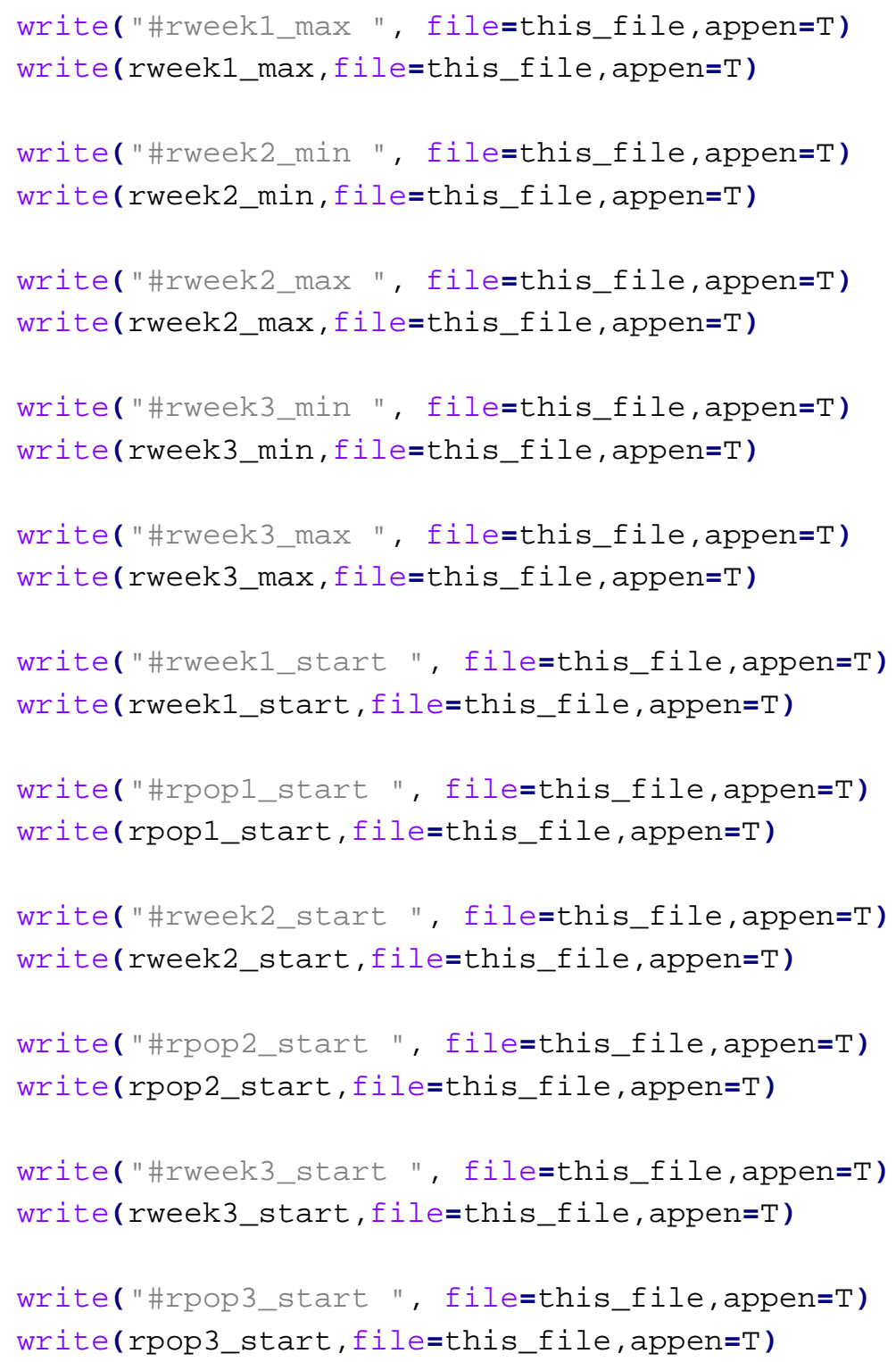


\#growth rates

growth_rates<-as.double (read.table (paste (WRITE_PATH, "squid_model.rep", sep=" ") , header $=\mathrm{F}, \operatorname{skip}=8, \operatorname{nrows}=1$ ))

optim_E<-as.double(read.table (paste(WRITE_PATH, "squid_model.rep", sep=" "), header $=\mathrm{F}, \operatorname{skip}=10$, nrows $=1$ ))

\#turn optim_E into a matrix

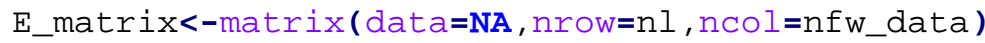

for ( $w$ in 1:nfw_data) \{

for ( 1 in $1: \mathrm{n} 1)$ (

E_matrix $[1, \mathrm{w}]<-o p t i m \_E\left[(\mathrm{w}-1) *_{\mathrm{n}} 1+1\right]$

了

\}

ylim<-max (E_matrix,proportions)

\#plot the optimised paramters against the data

$\operatorname{par}(\operatorname{mfrow}=\mathrm{C}(4,4), \operatorname{oma}=\mathrm{C}(0,0,0,0), \operatorname{mai}=\mathrm{c}(0.8,0.6,0.1,0.1), \mathrm{omd}=\mathrm{c}(0,0,0,0), \mathrm{omi}=\mathrm{c}$ $(0,0,0,0))$

\section{for ( $w$ in $\left.1: n f w \_d a t a\right) ~\{$}

this_week<-data_fishing_weeks [w]

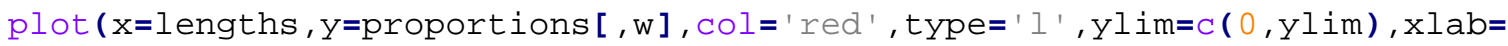
'length (cm)',ylab='proportion')

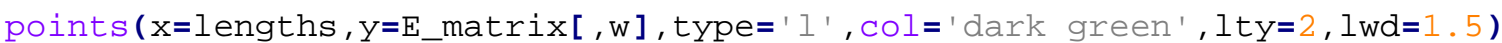

title (sub=paste ("week ",this_week, sep=" "))

\}

this_file<-paste(PLOT_PATH, "trial", tn, " LF", sep=" ")

savePlot (filename = this_file, type = "tif")

\#write optimised parameters, q, expoloitable biomass and catch proportions

to file

this_file<-paste(this_output_file, "trial",tn, ".txt", sep=" ")

write (optim_pars, file=this_file, ncolumns=length (optim_pars))

write (dep, file=this_file, ncolumns=naw, append=T)

\#final depletion

write (dep [naw], file=this_file, ncolumns=1, append=T)

write (q, file=this_file, ncolumns=1, append=T)

write (expB, file=this_file, ncolumns=naw, append=T)

write(growth_rates, file=this_file, ncolumns=nl, append=T)

write (optim_E, file=this_file, append=T, ncolumns=nfw_data)

\#select observed proportions at length data and title 0

or-proportions

\#\#\#calculate SCALING FACTOR, w, for SAMPLE SIZE N \#\#\#

\#calculate mean of estimated lengths for each week

E_mean<-rep $(0, \mathrm{nfw}$ _data)

for ( $w$ in $1: n f w \_$data) \{

for (l in $1: n 1)$ ( 


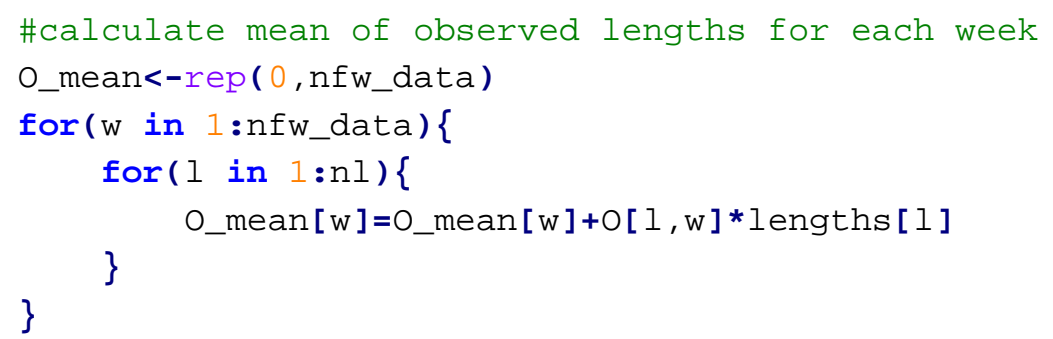




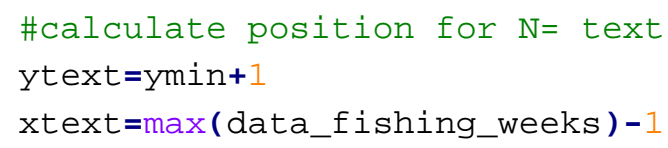

